\title{
Cellular Bases for Algebras with a Jones Basic Construction
}

\author{
John Enyang ${ }^{1}$ - Frederick M. Goodman ${ }^{2}$
}

Received: 28 August 2015 / Accepted: 20 June 2016 / Published online: 17 August 2016

(C) The Author(s) 2016. This article is published with open access at Springerlink.com

\begin{abstract}
We construct analogues for the Brauer, BMW, partition, and Jones-TemperleyLieb algebras of the Murphy basis of the Hecke algebra of the symmetric group. The bases are cellular bases indexed by paths on branching diagrams, and compatible with restriction of cell modules. The Jucys-Murphy elements for each class of algebras act by triangular matrices on the Murphy basis.
\end{abstract}

Keywords Cellular algebra · Jones basic construction · Murphy basis · Brauer algebra Birman-Murakami-Wenzl algebra $\cdot$ Partition algebra

Mathematics Subject Classification (2010) 20G05 · 05E10

\section{Introduction}

This paper develops analogues for the Brauer, BMW, partition, and Jones-Temperley-Lieb algebras of the Murphy basis [33] of the Iwahori-Hecke algebras $H_{n}\left(q^{2}\right)$ of the symmetric groups $\mathfrak{S}_{n}$.

The Murphy basis of $H_{n}\left(q^{2}\right)$ has many remarkable properties. First, it is a cellular basis in the sense of Graham and Lehrer [16]. For cellular algebras (i.e. algebras with a cellular basis) in general, one can define a family of modules known as cell modules. Then for any specialization of the cellular algebra over a field, all simple modules appear as quotients of the cell modules, and the algebra is semisimple exactly when all the cell modules are

Presented by Jon F. Carlson.

John Enyang

john.enyang.1@city.ac.uk

Frederick M. Goodman

frederick-goodman@uiowa.edu

1 Department of Mathematics, City University London, London, UK

2 Department of Mathematics, University of Iowa, Iowa City, IA, USA 
simple. The Murphy basis has a number of additional special properties. The Jucys-Murphy elements of the Hecke algebra act in a triangular fashion on the Murphy basis; this action allows the construction of the seminormal representations and the classification of simple modules and blocks, see [28], Chapter 3. Moreover, the Murphy basis is well adapted to the tower of Hecke algebras $\left(H_{n}\left(q^{2}\right)\right)_{n \geq 0}$, as will be explained below.

Several papers in the literature have aimed at generalizations or axiomatizations of the Murphy basis, the seminormal basis, and the set of Jucys-Murphy elements, for example $[9,14,29,37]$. The present paper is also a contribution to this theme.

In order to develop analogues of the Murphy basis for the Brauer algebras, etc., we first develop a new interpretation of the Murphy basis of the Hecke algebras $H_{n}\left(q^{2}\right)$. We begin with observing that the Hecke algebras $H_{n}=H_{n}\left(\boldsymbol{q}^{2}\right)$ defined over the generic ground ring $\mathbb{Z}\left[\boldsymbol{q}^{ \pm 1}\right]$, with $\boldsymbol{q}$ an indeterminant, have the following properties:

- The Hecke algebras $H_{n}$ are cyclic cellular algebras. This means that each cell module is a cyclic $H_{n}$-module.

- The cellular structures on the tower of algebras $H_{n}$ are coherent. This means that a cell module of $H_{n}$, restricted to the $H_{n-1}$ or induced to $H_{n+1}$ has a "cell filtration", that is, a filtration with cell modules as subquotients.

We therefore begin by studying coherent towers $\left(A_{n}\right)_{n \geq 0}$ of cyclic cellular algebras in general. We first obtain some rather simple general results about cellular bases in such towers, in Section 3. We observe that there exists a system of "branching factors" associated to each edge of the generic branching diagram for the tower. In fact, there is a system of " $d$-branching factors" related to the cell filtration of restricted cell modules, and a system of " $u$-branching factors" related to the cell filtration of induced modules. Then we note that an ordered product of $d$-branching factors along paths on the generic branching diagram determine bases of each cell module of each $A_{n}$ as well as a cellular basis of each $A_{n}$.

In Section 4, we consider the tower of Hecke algebras $H_{n}\left(\boldsymbol{q}^{2}\right)$. Recalling that this is a coherent tower of cyclic cellular algebras, we compute branching factors for reduced and induced cell modules. We show that the bases obtained via ordered products of $d$-branching factors coincide with the Murphy bases.

This construction could be regarded as a cellular analogue of the constructions in [34-36, 38].

Each of the examples that we want to study is a tower $\left(A_{n}\right)_{n \geq 0}$ of algebras that generically is obtained from another tower $\left(Q_{n}\right)_{n \geq 0}$ by repeated Jones basic constructions. For the Brauer, BMW, or partition algebras, the tower $\left(Q_{n}\right)_{n \geq 0}$ is a tower of Hecke algebras or symmetric group algebras. For the Jones-Temperley-Lieb algebras defined over a ring $R$, the tower $\left(Q_{n}\right)_{n \geq 0}$ is just the constant sequence $R$. Cellularity of such a tower $\left(A_{n}\right)_{n \geq 0}$ was previously studied in $[13,14]$. Here we augment the framework of those papers by the assumption that the algebras $Q_{n}$ are cyclic cellular. It follows easily from the previous work in $[13,14]$ that the tower $\left(A_{n}\right)_{n \geq 0}$ is a coherent tower of cyclic cellular algebras. We show here that branching factors, and therefore cellular bases for the tower $\left(A_{n}\right)_{n \geq 0}$ can be obtained by explicit formulas from branching factors for the tower $\left(Q_{n}\right)_{n \geq 0}$. These are our analogues of Murphy's bases.

Finally, in Section 5, we apply our results to the Brauer algebras, Birman-MurakamiWenzl (BMW) algebras, Jones-Temperley-Lieb algebras, and partition algebras, and obtain explicit Murphy bases for each of these families of algebras.

A complication in our approach to the Murphy bases is that the results of $[13,14]$ do not apply to Jones the basic construction algebras defined over their generic ground ring, say $R_{0}$, but only to the algebras defined over $R_{0}\left[\delta^{-1}\right]$, where $\delta$ is the "loop parameter"; see Section 5.1, where a mistake in $[13,14]$ is discussed and corrected. Therefore, the Murphy 
bases appear a priori to be bases only for the algebras defined over $R_{0}\left[\boldsymbol{\delta}^{-1}\right]$. However, as the bases are explicit, we can check for each of our examples that the Murphy basis is actually a basis for the algebras defined over the generic ground ring $R_{0}$. A considerable portion of the work in Section 5 is devoted to this final step. For the Jones-Temperley-Lieb algebras, our Murphy basis turns out to be none other than the usual diagram basis.

Our Murphy bases are "families of path bases", in the sense of Definition 3.8. It is known that for each of our examples, the tower $\left(A_{n}\right)_{n \geq 0}$ has a family of Jucys-Murphy elements in the sense of [14]. It follows from [14, Propositions 3.6 and 3.7] that the Jucys-Murphy elements act triangularly on our Murphy bases. Hence, Mathas' theory of cellular algebras with Jucys-Murphy elements and seminormal representations [29] can be applied. It is shown in [1] that triangularity actually holds with respect to dominance order, strengthening the triangularity statements of [13].

The Murphy bases for the partition algebras given in Theorem 6.26 have been used to obtain an analogue of the Young seminormal form for partition algebras in [10]. In the cases of the Brauer and BMW algebras, our results recover the Murphy type bases obtained in [9]; however, the construction here is simpler, and does not involve computations in the braid group. Rui and Si [37] used the path bases from [9] to compute Gram determinants for cell modules of the BMW algebras, and to obtain definitive semisimplicity results.

The Murphy bases of the (abstract) Brauer algebras are used in [2] to produce integral cellular bases of Brauer's centralizer algebras acting on symplectic or orthogonal tensor space. The analogous result holds also for the walled Brauer algebras acting on mixed tensor space $[2,38]$.

Murphy bases are used in [3] to construct skew cell modules of diagram algebras, which are analogous the the skew Specht modules of the symmetric groups.

There are many other examples of coherent towers of cellular algebras obtained by repeated Jones basic constructions, see [13]. Our method of obtaining explicit Murphy type bases as ordered products of branching factors has been applied to the walled Brauer algebras in [2] and should also apply to such examples as the cyclotomic BMW algebras.

\section{Preliminaries}

\subsection{Cellular Algebras}

Cellular algebras were defined by Graham and Lehrer [16]. In this paper we use a slightly weaker version of cellularity which was introduced in $[12,13]$.

Definition 2.1 Let $R$ be an integral domain. A cellular algebra is a tuple $(A, *, \widehat{A}, \unrhd, \mathscr{A})$ where

(1) $A$ is a unital $R$-algebra and $*: A \rightarrow A$ is an algebra involution, that is an $R$-linear anti-automorphism of $A$ such that $\left(x^{*}\right)^{*}=x$ for $x \in A$;

(2) $(\widehat{A}, \unrhd)$ is a finite partially ordered set, and $\widehat{A}^{\lambda}$, for $\lambda \in \widehat{A}$, is a finite indexing set;

(3) The set

$$
\mathscr{A}=\left\{c_{\mathfrak{s t}}^{\lambda} \mid \lambda \in \widehat{A} \text { and } \mathfrak{s}, \mathfrak{t} \in \widehat{A}^{\lambda}\right\}
$$

is an $R$-basis for $A$, for which the following conditions hold: 
(a) Given $\lambda \in \widehat{A}, \mathfrak{t} \in \widehat{A}^{\lambda}$, and $a \in A$, there exist coefficients $r_{\mathfrak{v}}(\mathfrak{t}, a) \in R$, for $\mathfrak{v} \in \widehat{A}^{\lambda}$, such that, for all $\mathfrak{s} \in \widehat{A}^{\lambda}$,

$$
c_{\mathfrak{s t}}^{\lambda} a \equiv \sum_{\mathfrak{v} \in \widehat{A}^{\lambda}} r_{\mathfrak{v}}(\mathfrak{t}, a) c_{\mathfrak{s v}}^{\lambda} \quad \bmod A^{\triangleright \lambda},
$$

where $A^{\triangleright \lambda}$ is the $R$-module generated by

$$
\left\{c_{\mathfrak{s t}}^{\mu} \mid \mu \in \widehat{A}, \mathfrak{s}, \mathfrak{t} \in \widehat{A}^{\mu} \text { and } \mu \triangleright \lambda\right\} .
$$

(b) If $\lambda \in \widehat{A}$ and $\mathfrak{s}, \mathfrak{t} \in \widehat{A}^{\lambda}$, then $\left(c_{\mathfrak{s t}}^{\lambda}\right)^{*} \equiv\left(c_{\mathfrak{t} \mathfrak{s}}^{\lambda}\right) \bmod A^{\triangleright \lambda}$.

The tuple $(A, *, \widehat{A}, \unrhd, \mathscr{A})$ is a cell datum for $A$.

If $A$ is an algebra with cell datum $(A, *, \widehat{A}, \unrhd, \mathscr{A})$ we will frequently omit reference to the cell datum for $A$ and simply refer to $A$ as a cellular algebra. The basis $\mathscr{A}$ is called a cellular basis of $A$.

From points 3(a) and 3(b) of the definition of cellularity, we have for $a \in A$ and $\mathfrak{s}, \mathfrak{t} \in \widehat{A}^{\lambda}$,

$$
a c_{\mathfrak{s t}}^{\lambda} \equiv \sum_{\mathfrak{v} \in \widehat{A}^{\lambda}} r_{\mathfrak{v}}\left(\mathfrak{s}, a^{*}\right) c_{\mathfrak{v} \mathfrak{t}}^{\lambda} \bmod A^{\triangleright \lambda} .
$$

An order ideal $\Gamma \subset \widehat{A}$ is a subset with the property that if $\lambda \in \Gamma$ and $\mu \unrhd \lambda$, then $\mu \in \Gamma$. It follows from the axioms of a cellular algebra that for any order ideal $\Gamma$ in $\widehat{A}$,

$$
A^{\Gamma}=\operatorname{span}\left\{c_{\mathfrak{s t}}^{\lambda} \mid \lambda \in \Gamma, \mathfrak{s}, \mathfrak{t} \in \widehat{A}^{\lambda}\right\}
$$

is a two sided ideal of $A$. In particular $A^{\triangleright \lambda}$ and

$$
A^{\unrhd \lambda}=\operatorname{span}\left\{c_{\mathfrak{s t}}^{\mu} \mid \mu \in \widehat{A}, \mathfrak{s}, \mathfrak{t} \in \widehat{A}^{\mu} \text { and } \mu \unrhd \lambda\right\}
$$

are two sided ideals.

Definition 2.2 Let $A$ be a cellular algebra, and $\lambda \in \widehat{A}$. The cell module $\Delta_{A}^{\lambda}$ is the right $A$-module defined as follows. As an $R$-module, $\Delta_{A}^{\lambda}$ is free with basis indexed by $\widehat{A}^{\lambda}$, say $\left\{c_{\mathfrak{t}}^{\lambda} \mid \mathfrak{t} \in \widehat{A}^{\lambda}\right\}$. The right $A$-action is given by

$$
c_{\mathfrak{t}}^{\lambda} a=\sum_{\mathfrak{v} \in \widehat{A}^{\lambda}} r_{\mathfrak{v}}(\mathfrak{t}, a) c_{\mathfrak{v}}^{\lambda},
$$

where the coefficients $r_{\mathfrak{v}}(\mathfrak{t}, a)$ are those of Eq. (2.1).

Thus, for any $\mathfrak{s} \in \widehat{A}^{\lambda}$, the map

$$
c_{\mathfrak{t}}^{\lambda} \mapsto c_{\mathfrak{s t}}^{\lambda}+A^{\triangleright \lambda}
$$

is an injective $A$-module homomorphism of the cell module $\Delta_{A}^{\lambda}$ into $A^{\unrhd \lambda} / A^{\triangleright \lambda}$.

If $A$ is an $R$-algebra with involution $*$, then $*$ induces functors $M \rightarrow M^{*}$ interchanging left and right $A$-modules, and taking $A-A$ bimodules to $A-A$ bimodules. We identify $M^{* *}$ with $M$ via $x^{* *} \mapsto x$ and for modules ${ }_{A} M$ and $N_{A}$ we have $\left(M \otimes_{R} N\right)^{*} \cong N^{*} \otimes_{R} M^{*}$, as $A-A$ bimodules, with the isomorphism determined by $(m \otimes n)^{*} \mapsto n^{*} \otimes m^{*}$. For a right $A-$ module $M_{A}$, using both of these isomorphisms, we identify $\left(M^{*} \otimes M\right)^{*}$ with $M^{*} \otimes M^{* *}=$ $M^{*} \otimes M$, via $\left(x^{*} \otimes y\right)^{*} \mapsto y^{*} \otimes x$. Now we apply these observations with $A$ a cellular algebra and $\Delta_{A}^{\lambda}$ a cell module. The assignment

$$
\alpha_{\lambda}: c_{\mathfrak{s t}}^{\lambda}+A^{\triangleright \lambda} \mapsto\left(c_{\mathfrak{s}}^{\lambda}\right)^{*} \otimes\left(c_{\mathfrak{t}}^{\lambda}\right)
$$


determines an $A-A$ bimodule isomorphism from $A^{\unrhd \lambda} / \mathrm{A}^{\triangleright \lambda}$ to $\left(\Delta_{A}^{\lambda}\right)^{*} \otimes_{R} \Delta_{A}^{\lambda}$. Moreover, we have $* \circ \alpha_{\lambda}=\alpha_{\lambda} \circ *$, which reflects the cellular algebra axiom $\left(c_{\mathfrak{s t}}^{\lambda}\right)^{*} \equiv c_{\mathfrak{t} \mathfrak{s}}^{\lambda} \bmod A^{\triangleright \lambda}$. When it is necessary to identify the algebra we are working with, we will write $\alpha_{\lambda}^{A}$ instead of $\alpha_{\lambda}$. The importance of the maps $\alpha_{\lambda}$ for the structure of cellular algebras was stressed by König and Xi [23, 24].

\subsection{Generic Ground Rings}

The most important examples of cellular algebras are actually families $A^{S}$ of algebras defined over various integral ground rings $S$, possibly containing distinguished elements (parameters) which enter into the definition of the algebras. The prototypical example is the Iwahori-Hecke algebra of the symmetric group $\mathcal{H}_{k}=\mathcal{H}_{k}\left(q^{2}\right)$, which can be defined over any integral domain $S$ with a distinguished invertible element $q$; see Section 4.2 for the detailed description.

Again in the most important examples, there is a "generic ground ring" $R$ for $A$ with the following properties:

(1) For any integral ground ring $S$ there is a ring homomorphism from $R$ to $S$, and the algebra over $S$ is the specialization of the algebra over $R$, that is $A^{S} \cong A^{R} \otimes_{R} S$. Likewise, the cell modules of $A^{S}$ are specializations of those of $A^{R}$, that is $\Delta_{A^{S}}^{\lambda} \cong$ $\Delta_{A^{R}}^{\lambda} \otimes_{R} S$.

(2) $R$ has characteristic zero, and if $F$ denotes the field of fractions of $R$, then $A^{F}$ is split semisimple; and the cell modules $\Delta_{A^{F}}^{\lambda}$ are the simple $A^{F}$ modules.

For example, the generic ground ring for the Iwahori-Hecke algebra is $\mathbb{Z}\left[\boldsymbol{q}, \boldsymbol{q}^{-1}\right]$, where $\boldsymbol{q}$ is an indeterminant over $\mathbb{Z}$.

Indeed, the entire point of the theory of cellular algebras is to provide a setting for a modular representation theory of important classes of algebras such as the Iwahori-Hecke algebras, Brauer algebras, Birman Murakami Wenzl algebras, etc. The cell modules of $A^{R}$ are integrally defined versions of the simple modules of $A^{F}$ which specialize to $A^{k}$-modules for any field $k$ (with appropriate parameters). The simple $A^{k}$ modules are found as quotients of the cell modules $\Delta_{A^{k}}^{\lambda}$. See $[16,28]$ for details.

\subsection{Equivalent Cellular Bases}

A cellular algebra $A$ with cell datum $(A, *, \widehat{A}, \unrhd, \mathscr{A})$ always admits different cellular bases $\mathscr{B}$. In fact, any choice of an $R$-basis in each cell module of $A$ can be globalized to a cellular basis of $A$, see Lemma 2.3. We say that a cellular basis

$$
\mathscr{B}=\left\{b_{\mathfrak{s t}}^{\lambda} \mid \lambda \in \widehat{A} \text { and } \mathfrak{s}, \mathfrak{t} \in \widehat{A}^{\lambda}\right\}
$$

is equivalent to the original cellular basis $\mathscr{A}$ if it determines the same ideals $A \unrhd \lambda$ and yields isomorphic cell modules.

Lemma 2.3 ([11], Lemma 2.3) Let $A$ be a cellular algebra with cell datum $(A, *, \widehat{A}, \unrhd, \mathscr{A})$. For each $\lambda \in \widehat{A}$, fix an $A-A$-bimodule isomorphism $\alpha_{\lambda}: A^{\unrhd \lambda} / A^{\triangleright \lambda} \rightarrow$ $\left(\Delta_{A}^{\lambda}\right)^{*} \otimes_{R} \Delta_{A}^{\lambda}$ satisfying $* \circ \alpha_{\lambda}=\alpha_{\lambda} \circ *$. For each $\lambda \in \widehat{A}$, let $\left\{b_{\mathfrak{t}} \mid \mathfrak{t} \in \widehat{A}^{\lambda}\right\}$ be an $R$-basis of the cell module $\Delta_{A}^{\lambda}$. For each $\lambda \in \widehat{A}$ and each $\mathfrak{s}, \mathfrak{t} \in \widehat{A}^{\lambda}$, let $b_{\mathfrak{s t}}^{\lambda}$ be a lifting of $\alpha_{\lambda}^{-1}\left(b_{\mathfrak{s}}^{*} \otimes b_{\mathfrak{t}}\right)$ 
in $A^{\unrhd \lambda}$. Then

$$
\mathscr{B}=\left\{b_{\mathfrak{s t}}^{\lambda} \mid \lambda \in \widehat{A} \text { and } \mathfrak{s}, \mathfrak{t} \in \widehat{A}^{l} a\right\}
$$

is a cellular basis of A equivalent to the original cellular basis $\mathscr{A}$.

\subsection{Extensions of Cellular Algebras}

Definition 2.4 Suppose $A$ is a unital $R$-algebra with involution $*$, and $J$ is an $*$-invariant ideal. Let us say that $J$ is a cellular ideal in $A$ if it satisfies the axioms for a cellular algebra (except for being unital) with cellular basis $\left\{c_{\mathfrak{s t}}^{\lambda} \mid \lambda \in \widehat{J}\right.$ and $\left.\mathfrak{s}, \mathfrak{t} \in \widehat{J}^{\lambda}\right\} \subseteq J$ and we have, as in point (3a) of the definition of cellularity,

$$
c_{\mathfrak{s t}}^{\lambda} a \equiv \sum_{v} r_{\mathfrak{v}}(\mathfrak{t}, a) c_{\mathfrak{s v}}^{\lambda} \quad \bmod J^{\triangleright \lambda} \text { for all } a \in A,
$$

not only for $a \in J$.

Lemma 2.5 (Extensions of cellular algebras) Let A be an algebra with involution over an integral domain $R$. Suppose that $J$ is a cellular ideal in A and A/J is a cellular algebra with the involution induced from $A$. Then $A$ is a cellular algebra.

Remark 2.6 Write $H=A / J$. Denote the cell datum of $J$ by $(J, *, \unrhd, \widehat{J}, \mathscr{J})$ and that of $H$ by $(H, *, \unrhd, \widehat{H}, \mathscr{H})$. Let $\pi: A \rightarrow A / J=H$ be the canonical map. The partially ordered set in the cell datum of $A$ is $\widehat{A}=\widehat{J} \cup \widehat{H}$, with the original partial orders on $\widehat{J}$ and $\widehat{H}$ and $\mu \triangleright \lambda$ for all $\mu \in \widehat{J}$ and $\lambda \in \widehat{H}$. If $\widetilde{\mathscr{H}}$ is any lifting of $\mathscr{H}$ in $A$, then $\mathscr{A}=\mathscr{J} \cup \widetilde{\mathscr{H}}$ is a cellular basis of $A$.

For $\lambda \in \widehat{H}$, the following statements hold: $A^{\unrhd \lambda}=\pi^{-1}\left(H^{\unrhd \lambda}\right)$, and likewise $A^{\triangleright \lambda}=$ $\pi^{-1}\left(H^{\triangleright \lambda}\right)$. Consequently, $J \subseteq A^{\triangleright \lambda}$ for all $\lambda \in \widehat{H}$. We have $A^{\unrhd \lambda} / A^{\triangleright \lambda} \cong H^{\unrhd \lambda} / H^{\triangleright \lambda}$ via $a+A^{\triangleright \lambda} \mapsto \pi(a)+H^{\triangleright \lambda}$. The cell modules $\Delta_{A}^{\lambda}$ and $\Delta_{H}^{\lambda}$ can be identified (by $x a=x \pi(a)$ for $x \in \Delta_{H}^{\lambda}$ and $\left.a \in A\right)$. The map $\alpha_{\lambda}^{A}: A^{\unrhd \lambda} / A^{\triangleright \lambda} \rightarrow\left(\Delta_{A}^{\lambda}\right)^{*} \otimes_{R} \Delta_{A}^{\lambda}$ is

$$
\alpha_{\lambda}^{A}: a+A^{\triangleright \lambda} \mapsto \alpha_{\lambda}^{H}\left(\pi(a)+H^{\triangleright \lambda}\right) .
$$

For $\mu \in \widehat{J}$, the cell modules $\Delta_{A}^{\mu}$ and $\Delta_{J}^{\mu}$ can be identified; this is because of condition (2.2) in the definition of cellular ideals. We have $A^{\unrhd \mu}=J \unrhd \mu \subseteq J$, and similarly for $A^{\triangleright \mu}$.

\subsection{Cellular Algebras with Cyclic Cell Modules}

Definition 2.7 A cellular algebra is said to be cyclic cellular if every cell module is cyclic.

Remark 2.8 For examples of cyclic cellular algebras, see Section 6. Cyclic cellularity was also introduced in [11], and some additional examples, beyond those studied here are presented in that paper.

Lemma 2.9 ([11], Lemma 2.5) Let $A$ be a cellular algebra with cell datum $(A, *, \widehat{A}, \unrhd, \mathscr{A})$. The following are equivalent:

(1) A is cyclic cellular.

(2) For each $\lambda \in \widehat{A}$, there exists an element $c_{\lambda} \in A^{\unrhd \lambda}$ with the properties:

(a) $c_{\lambda} \equiv c_{\lambda}^{*} \bmod A^{\triangleright \lambda}$.

(b) $A^{\unrhd \lambda}=A c_{\lambda} A+A^{\triangleright \lambda}$. 
(c) $\left(c_{\lambda} A+A^{\triangleright \lambda}\right) / A^{\triangleright \lambda} \cong \Delta_{A}^{\lambda}$, as right A-modules.

For a cyclic cellular algebra $A$ and $\lambda \in \widehat{A}$, we let $\delta_{A}^{\lambda}$ denote a generator of the cell module $\Delta_{A}^{\lambda}$. The element $c_{\lambda}$ in Lemma 2.9 can be taken to be any lifting to $A^{\unrhd \lambda}$ of $\alpha^{-1}\left(\left(\delta_{A}^{\lambda}\right)^{*} \otimes \delta_{A}^{\lambda}\right)$. When it is necessary to identify the algebra we are working in, we write $c_{\lambda}^{A}$.

We record a version of Lemma 2.3 that is adapted to the context of cyclic cellular algebras:

Lemma 2.10 Let $A$ be a cyclic cellular algebra with cell datum $(A, *, \widehat{A}, \unrhd, \mathscr{A})$. For each $\lambda \in \widehat{A}$, let $\left\{b_{\mathfrak{t}} \mid \mathfrak{t} \in \widehat{A}^{\lambda}\right\}$ be an $R$-basis of $\Delta_{A}^{\lambda}$. For $\mathfrak{t} \in \widehat{A}^{\lambda}$, choose $w_{\mathfrak{t}} \in A$ such that $b_{\mathfrak{t}}=\delta_{A}^{\lambda} w_{\mathfrak{t}}$. For $\mathfrak{s}, \mathfrak{t} \in \widehat{A}^{\lambda}$, let

$$
b_{\mathfrak{s t}}^{\lambda}=\left(w_{\mathfrak{s}}\right)^{*} c_{\lambda} w_{\mathfrak{t}}
$$

Then $\mathscr{B}=\left\{b_{\mathfrak{s t}}^{\lambda} \mid \lambda \in \widehat{A}\right.$ and $\left.\mathfrak{s}, \mathfrak{t} \in \widehat{A}^{\lambda}\right\}$ is a cellular basis of A equivalent to the original cellular basis $\mathscr{A}$.

Proof For each $\lambda \in \widehat{A}$ and $\mathfrak{s}, \mathfrak{t} \in \widehat{A}^{\lambda}, b_{\mathfrak{s t}}^{\lambda}$ is a lifting in $A^{\unrhd \lambda}$ of $\alpha_{\lambda}^{-1}\left(b_{\mathfrak{s}}^{*} \otimes b_{\mathfrak{t}}\right)$, so this follows immediately from Lemma 2.3 .

Remark 2.11 (Extensions of cyclic cellular algebras) Let $A$ be an algebra with involution over $R$, let $J$ be a cellular ideal in $A$ and suppose that $H=A / J$ is cellular. If both $J$ and $H$ are cyclic cellular, then so is $A$. This is evident from Lemma 2.5 and Remark 2.6.

Let $\pi: A \rightarrow A / J=H$ denote the quotient map. For each $\lambda \in \widehat{H}$, let $\delta_{H}^{\lambda}$ be a generator of the cell module $\Delta_{H}^{\lambda}=\Delta_{A}^{\lambda}$. Let $c_{\lambda}^{H} \in H^{\unrhd \lambda}$ satisfy $\alpha_{\lambda}^{H}\left(c_{\lambda}^{H}+H^{\triangleright \lambda}\right)=\left(\delta_{H}^{\lambda}\right)^{*} \otimes \delta_{H}^{\lambda}$. Then $c_{\lambda}^{A}$ can be taken to be any element of $\pi^{-1}\left(c_{\lambda}^{H}\right)$.

\section{Bases in Towers of Cellular Algebras}

In this section we obtain some elementary results on bases in towers of cellular algebras. The main results can be summarized as follows. Consider an increasing sequence of cellular algebras $\left(H_{n}\right)_{n \geq 0}$ over an integral domain $R$ with field of fractions $F$. Suppose that

(1) $H_{0}=R$, and $H_{n}^{F}=H_{n} \otimes_{R} F$ is split semisimple for all $n$.

(2) For each $n \geq 0$ and each cell module $\Delta$ of $H_{n+1}$, the $H_{n}$-module $\operatorname{Res}_{H_{n}}^{H_{n+1}}(\Delta)$ has an order preserving cell filtration, see Definition 3.1

(3) $H_{n}$ is cyclic cellular for all $n$.

Then one can associate to each edge $\lambda \rightarrow \mu$ in the branching diagram $\widehat{H}$ for the tower $\left(H_{N}^{F}\right)_{n \geq 0}$ of split semisimple algebras a "branching factor" $d_{\lambda \rightarrow \mu}$. The ordered product of these branching factors along paths in $\widehat{H}$ determines a basis of each cell module of each algebra $H_{n}$. The collection of these bases is a "family of path bases," which means that the bases behave well with respect to restriction to smaller algebras in the tower, see Definition 3.8. The existence of these special bases of the cell modules depends on the existence of cell filtrations for the restricted modules $\operatorname{Res}_{H_{n}}^{H_{n+1}}(\Delta)$; conversely, any family of path bases

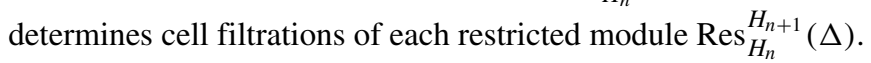




\subsection{Coherence Conditions for Towers of Cellular Algebras}

If $A$ is a cellular algebra over $R, \lambda \in \widehat{A}$, and $N \subseteq M$ is an inclusion of right $A$-modules, write $N \stackrel{\lambda}{\subseteq} M$ if $M / N \cong \Delta_{A}^{\lambda}$ as right $A$-modules.

Definition 3.1 Let $A$ be a cellular algebra with cell datum $(A, *, \unrhd, \widehat{A}, \mathscr{A})$. If $M$ is a right $A$-module, a cell filtration of $M$ is a filtration by right $A$-modules

$$
\{0\}=M_{0} \stackrel{\lambda^{(1)}}{\subseteq} M_{1} \stackrel{\lambda^{(2)}}{\subseteq} \cdots \stackrel{\lambda^{(r)}}{\subseteq} M_{r}=M,
$$

with subquotients isomorphic to cell modules. Say that the filtration is order preserving if $\lambda^{(s)} \triangleright \lambda^{(t)}$ in $\widehat{A}$ whenever $s<t$.

Observe that all the modules occurring in a cell filtration are necessarily free as $R-$ modules. Evidently, a given cell module occurs at most once as a subquotient in an order preserving cell filtration.

Here and in the remainder of the paper, we will consider increasing sequences

$$
H_{0} \subseteq H_{1} \subseteq H_{2} \cdots
$$

of cellular algebras over an integral domain $R$. Whenever we have such a sequence of algebras, we assume that all the inclusions are unital and that the involutions are consistent; that is the involution on $H_{i+1}$, restricted to $H_{i}$, agrees with the involution on $H_{i}$.

Definition 3.2 $([13,14])$ The tower of cellular algebras $\left(H_{i}\right)_{i \geq 0}$ is coherent if the following conditions are satisfied:

(1) For each $i \geq 0$ and each cell module $\Delta$ of $H_{i}$, the induced module $\operatorname{Ind}_{H_{i}}^{H_{i+1}}(\Delta)$ has cell filtration.

(2) For each $i \geq 0$ and each cell module $\Delta$ of $H_{i+1}$ the restricted module $\operatorname{Res}_{H_{i}}^{H_{i+1}}(\Delta)$ has a cell filtration.

The tower is called strongly coherent if the cell filtrations can be chosen to be order preserving.

In the examples of interest to us, we will also have uniqueness of the multiplicities of the cell modules appearing as subquotients of the cell filtrations, and Frobenius reciprocity connecting the multiplicities in the two types of filtrations, see Corollary 3.5.

Only the filtrations of restricted modules $\operatorname{Res}_{H_{i}}^{H_{i+1}}(\Delta)$ play a role in this section, but the filtrations of induced modules $\operatorname{Ind}_{H_{i}}^{H_{i+1}}(\Delta)$ also play an essential role in the study of towers of algebras with a Jones basic construction in Sections 5 and 6.

\subsection{Inclusions Matrices, Branching Diagrams, and Cell Filtrations}

We recall the notion of an inclusion matrix for an inclusion of split semisimple algebras over a field. Suppose $A \subseteq B$ are finite dimensional split semisimple algebras over a field $F$ (with the same identity element). Let $\left\{V_{\lambda} \mid \lambda \in \widehat{A}\right\}$, be the set of isomorphism classes of simple $A$-modules and $\left\{W_{\mu} \mid \mu \in \widehat{B}\right\}$ the set of isomorphism classes of simple $B$-modules. We associate a $\widehat{B} \times \widehat{A}$ inclusion matrix $\omega$ to the inclusion $A \subseteq B$, as follows. 
For each $\mu \in \widehat{B}$ and $\lambda \in \widehat{A}$, define $\omega(\mu, \lambda)$ to be the multiplicity of $V_{\lambda}$ in a direct sum decomposition of $\operatorname{Res}_{A}^{B}\left(W_{\mu}\right)$. Say that the inclusion $A \subseteq B$ is multiplicity-free if the inclusion matrix has entries in $\{0,1\}$.

Now consider an increasing sequence $\left(B_{n}\right)_{n \geq 0}$ of split semisimple algebras over a field $F$. Suppose that all the inclusions $B_{n} \subseteq B_{n+1}$ are multiplicity-free. (This suffices for the examples we want to treat). The branching diagram $\widehat{B}$ of the sequence $\left(B_{n}\right)_{n>0}$ is a graph with vertex set $\bigsqcup_{n \geq 0} \widehat{B}_{n}$, where $\widehat{B}_{n}$ indexes the set of isomorphism classes simple $B_{n}-$ modules. Fix $n \geq 0$ and let $\omega_{n}$ denote the inclusion matrix of $B_{n} \subseteq B_{n+1}$. For $\lambda \in \widehat{B}_{n}$ and $\mu \in \widehat{B}_{n+1}$, the branching diagram has a unique edge connecting $\lambda$ and $\mu$ if $\omega_{n}(\mu, \lambda) \neq 0$. In this case, we write $\lambda \rightarrow \mu$. In our examples we have $B_{0}=F$, so that $\widehat{B}_{0}$ is a singleton.

Notation 3.3 Let $R$ be an integral domain with field of fractions $F$. Let $A$ be a cellular algebra over $R$ and $\Delta$ an $A$-module. Write $A^{F}$ for $A \otimes_{R} F$ and $\Delta^{F}$ for $\Delta \otimes_{R} F$.

Let $A$ be a cellular algebra over an integral domain $R$ with field of fractions $F$, and suppose $A^{F}$ is split semisimple. Then $\left\{\left(\Delta_{A}^{\lambda}\right)^{F} \mid \lambda \in \widehat{A}\right\}$ is a complete family of simple $A^{F}$-modules.

Lemma 3.4 ([13, Lemma 2.22] and [14, Sect. 2.5]) Let $R$ be an integral domain with field of fractions $F$. Suppose that $A \subseteq B$ are cellular algebras over $R$ and that $A^{F}$ and $B^{F}$ are split semisimple. Let $\omega$ denote the inclusion matrix for $A^{F} \subseteq B^{F}$.

1. For any $\lambda \in \widehat{A}$ and $\mu \in \widehat{B}$, and any cell filtration of $\operatorname{Res}_{A}^{B}\left(\Delta_{B}^{\mu}\right)$, the number of subquotients of the filtration isomorphic to $\Delta_{A}^{\lambda}$ is $\omega(\mu, \lambda)$.

2. Likewise, for any $\lambda \in \widehat{A}$ and $\mu \in \widehat{B}$, and any cell filtration of $\operatorname{Ind}_{A}^{B}\left(\Delta_{A}^{\lambda}\right)$, the number of subquotients of the filtration isomorphic to $\Delta_{B}^{\mu}$ is $\omega(\mu, \lambda)$.

Corollary 3.5 Let $R$ be an integral domain with field of fractions $F$. Let $\left(H_{n}\right)_{n \geq 0}$ be a strongly coherent tower of cellular algebras over $R$, and suppose that $H_{n}^{F}$ is split semisimple for all $n$. Then for all $n$ and for $\lambda \in \widehat{H}_{n}$ and $\mu \in \widehat{H}_{n+1}$, the multiplicity of $\Delta_{H_{n}}^{\lambda}$ in any cell filtration of $\operatorname{Res}_{H_{n}}^{H_{n+1}}\left(\Delta_{H_{n+1}}^{\mu}\right)$ equals the multiplicity of $\Delta_{H_{n+1}}^{\mu}$ in any cell filtration of $\operatorname{Ind}_{H_{n}}^{H_{n+1}}\left(\Delta_{H_{n}}^{\lambda}\right)$. The multiplicities are independent of the choice of the filtrations.

\subsection{Path Bases and Cell Filtrations}

We consider an increasing sequence $\left(H_{n}\right)_{n \geq 0}$ of cellular algebras over an integral domain $R$ with field of fractions $F$. We assume the following conditions are satisfied:

(1) $H_{0}=R$, and $H_{n}^{F}$ is split semisimple for all $n$.

(2) The branching diagram $\widehat{H}$ for the tower $\left(H_{n}^{F}\right)_{n \geq 0}$ is multiplicity free.

We let $\left(H_{n}, *, \unrhd, \widehat{H}_{n}, \mathscr{H}_{n}\right)$ denote a cell datum for $H_{n}$. Denote the unique element of $\widehat{H}_{0}$ by $\emptyset$.

Definition 3.6 A path on $\widehat{H}$ from $\lambda \in \widehat{H}_{l}$ to $\mu \in \widehat{H}_{m}(l<m)$ is a sequence

$$
\left(\lambda=\lambda^{(l)}, \lambda^{(l+1)}, \ldots, \lambda^{(m)}=\mu\right)
$$

with $\lambda^{(i)} \in \widehat{H}_{i}$ and $\lambda^{(i)} \rightarrow \lambda^{(i+1)}$ for all $i$. A path $\mathfrak{s}$ from $\lambda$ to $\mu$ and a path $\mathfrak{t}$ from $\mu$ to $v$ can be concatenated in the obvious way; denote the concatenation by $\mathfrak{s} \circ \mathfrak{t}$. If $\mathfrak{t}=(\emptyset=$ 
$\left.\lambda^{(0)}, \lambda^{(1)}, \ldots, \lambda^{(n)}=\lambda\right)$ is a path from $\emptyset$ to $\lambda \in \widehat{H}_{n}$, and $0 \leq k<l \leq n$, write $\mathfrak{t}(k)=\lambda^{(k)}$, $\mathfrak{t}_{[k, l]}$ for the path $\left(\lambda^{(k)}, \ldots, \lambda^{(l)}\right)$, and write $\mathfrak{t}^{\prime}$ for $\mathfrak{t}_{[0, n-1]}$.

Since for all $n \geq 0$ and all $\mu \in \widehat{H}_{n}$, the rank of the cell module $\Delta_{H_{n}}^{\mu}$ equals the dimension over $F$ of $\left(\Delta_{H_{n}}^{\mu}\right)^{F}$, and the latter is the number of paths on the branching diagram $\widehat{H}$ from $\emptyset$ to $\mu$, we can take $\widehat{H}_{n}^{\mu}$ to be the set of such paths.

We define a total order on paths on $\widehat{H}$ as follows:

Definition 3.7 Let $\mathfrak{s}=\left(\lambda^{(l)}, \lambda^{(l+1)}, \ldots, \lambda^{(m)}\right)$ and $\mathfrak{t}=\left(\mu^{(l)}, \mu^{(l+1)}, \ldots, \mu^{(m)}\right)$ be two paths from $\widehat{H}_{l}$ to $\widehat{H}_{m}$. Say that $\mathfrak{s}$ precedes $\mathfrak{t}$ in reverse lexicographic order (denoted $\mathfrak{s} \preceq \mathfrak{t}$ ) if $\mathfrak{s}=\mathfrak{t}$, or if for the last index $j$ such that $\lambda^{(j)} \neq \mu^{(j)}$, we have $\lambda^{(j)}<\mu^{(j)}$ in $\widehat{H}_{j}$.

Definition 3.8 ([14]) For each $n \geq 0$ and each $\lambda \in \widehat{H}_{n}$, let $\left\{b_{\mathfrak{t}}^{\lambda} \mid \mathfrak{t} \in \widehat{H}_{n}^{\lambda}\right\}$ be a basis of the cell module $\Delta_{H_{n}}^{\lambda}$. The family of bases is called a family of path bases if the following condition holds: Let $\lambda \in \widehat{H}_{n}$ and let $\mathfrak{t} \in \widehat{H}_{n}^{\lambda}$. Fix $k<n$ and write $\mathfrak{t}_{1}=\mathfrak{t}_{[0, k]}$, and $\mathfrak{t}_{2}=\mathfrak{t}_{[k, n]}$, and $\mu=\mathfrak{t}(k)$. Let $x \in H_{k}$, and let $b_{\mathfrak{t}_{1}}^{\mu} x=\sum_{\mathfrak{s}} r\left(x ; \mathfrak{s}, \mathfrak{t}_{1}\right) b_{\mathfrak{s}}^{\mu}$. Then

$$
b_{\mathfrak{t}}^{\lambda} x \equiv \sum_{\mathfrak{s}} r\left(x ; \mathfrak{s}, \mathfrak{t}_{1}\right) b_{\mathfrak{s o t}}^{\lambda},
$$

$\operatorname{modulo~} \operatorname{span}\left\{b_{\mathfrak{v}}^{\lambda} \mid \mathfrak{v}_{[k, n]} \succ \mathfrak{t}_{[k, n]}\right\}$

\section{Lemma 3.9}

(1) Suppose that for all $n \geq 1$ and for all $\mu \in \widehat{H}_{n}$, $\operatorname{Res}_{H_{n-1}}^{H_{n}}\left(\Delta_{H_{n}}^{\mu}\right)$ has an order preserving cell filtration. Then the cell modules of the tower $\left(H_{n}\right)_{n \geq 0}$ have a family of path bases.

(2) Conversely, suppose we are given a family of path bases of the cell modules of the tower $\left(H_{n}\right)_{n \geq 0}$. Then for all $n \geq 1$ and for all $\mu \in \widehat{H}_{n}$, $\operatorname{Res}_{H_{n-1}}^{H_{n}}\left(\Delta_{H_{n}}^{\mu}\right)$ has a cell filtration. Moreover, if $\left\{\lambda \in \widehat{H}_{n-1} \mid \lambda \rightarrow \mu\right\}$ is totally ordered in $\widehat{H}_{n-1}$, then $\operatorname{Res}_{H_{n-1}}^{H_{n}}\left(\Delta_{H_{n}}^{\mu}\right)$ has an order preserving cell filtration.

Proof The first statement is proved in [14, Proposition 2.18]; we will give more concrete construction of path bases in Section 3.5, in the case that all the algebras $H_{n}$ are cyclic cellular.

For the converse, suppose we are given a family of path bases $\left\{b_{\mathfrak{t}} \mid \mathfrak{t} \in \widehat{H}_{n}^{\mu}\right\}$ for $n \geq 0$ and for $\mu \in \widehat{H}_{n}$. For $n \geq 1$ and $\mu \in \widehat{H}_{n}$, let $\lambda^{(1)}, \ldots, \lambda^{(s)}$ be a list of $\left\{\lambda \in \widehat{H}_{n-1} \mid \lambda \rightarrow \mu\right\}$, ordered so that $i<j$ if $\lambda^{(i)} \triangleright \lambda^{(j)}$. Let

$$
N_{j}=\operatorname{span}\left\{b_{\mathfrak{t}} \mid \mathfrak{t} \in \widehat{H}_{n}^{\mu} \text { and } \mathfrak{t}(n-1)=\lambda^{(i)} \text { for some } i \leq j\right\} .
$$

It follows from the definition of a path basis that $N_{j}$ is an $H_{n-1}$ submodule of $\operatorname{Res}_{H_{n-1}}^{H_{n}}\left(\Delta_{H_{n}}^{\mu}\right)$, and that $N_{j} / N_{j-1} \cong \Delta_{H_{n-1}}^{\lambda^{(j)}}$. If $\left\{\lambda \in \widehat{H}_{n-1} \mid \lambda \rightarrow \mu\right\}$ is totally ordered, then clearly this cell filtration is order preserving.

\subsection{Cyclic Cellularity and Branching Factors}

Suppose that $A \subseteq B$ are cyclic cellular algebras over an integral domain $R$. We have the following observations regarding cell filtrations of restricted and induced modules: 
(1) Let $\mu \in \widehat{B}$ and suppose that $\operatorname{Res}_{A}^{B}\left(\Delta_{B}^{\mu}\right)$ has a cell filtration:

$$
\{0\}=M_{0} \stackrel{\lambda^{(1)}}{\subseteq} M_{1} \stackrel{\lambda^{(2)}}{\subseteq} \cdots \stackrel{\lambda^{(r)}}{\subseteq} M_{r}=\operatorname{Res}_{A}^{B}\left(\Delta_{B}^{\mu}\right) .
$$

Let $\delta_{B}^{\mu}$ be a generator of the $B$-module $\Delta_{B}^{\mu}$. Since $M_{j} / M_{j-1} \cong \Delta_{A}^{\lambda^{(j)}}$ is a cyclic $A$ module, there exists an element $d_{\lambda^{(j)} \rightarrow \mu}^{B} \in B$ such that $\delta_{B}^{\mu} d_{\lambda^{(j)} \rightarrow \mu}^{B}+M_{j-1}$ is a generator of $M_{j} / M_{j-1}$.

(2) Let $\lambda \in \widehat{A}$ and suppose $\operatorname{Ind}_{A}^{B}\left(\Delta_{A}^{\lambda}\right)$ has a cell filtration:

$$
\{0\}=N_{0} \stackrel{\mu^{(1)}}{\subseteq} N_{1} \stackrel{\mu^{(2)}}{\subseteq} \cdots \stackrel{\mu^{(p)}}{\subseteq} N_{p}=\operatorname{Ind}_{A}^{B}\left(\Delta_{A}^{\lambda}\right) .
$$

Let $\delta_{A}^{\lambda}$ be a generator of the $A$-module $\Delta_{A}^{\lambda}$; then $\delta_{A}^{\lambda} \otimes 1$ is a generator of the $B$-module $\operatorname{Ind}_{A}^{B}\left(\Delta_{A}^{\lambda}\right)$. Since $N_{j} / N_{j-1} \cong \Delta_{B}^{\mu^{(j)}}$ is a cyclic $B$-module, there exists an element $u_{\lambda \rightarrow \mu^{(j)}}^{B} \in B$ such that $\delta_{A}^{\lambda} \otimes u_{\lambda \rightarrow \mu^{(j)}}^{B}+N_{j-1}$ is a generator of $N_{j} / N_{j-1}$.

We call the elements $d_{\lambda \rightarrow \mu}^{B}$ and $u_{\lambda \rightarrow \mu}^{B}$ branching factors. They are not canonical, but in the examples in Sections 4 and 6, it will be possible to make natural choices for these elements.

\subsection{Bases of Cell Modules in Towers of Cyclic Cellular Algebras}

Consider a tower $\left(H_{n}\right)_{n \geq 0}$ of cellular algebras over an integral domain $R$ with field of fractions $F$, satisfying conditions (3)-(3) listed at the beginning of Section 3 We let $\left(H_{n}, *, \unrhd, \widehat{H}_{n}, \mathscr{H}_{n}\right)$ denote a cell datum for $H_{n}$. Denote by $\emptyset$ the unique element of $\widehat{H}_{0}$.

Because of assumptions (3)-(3), and Lemma 3.4, there is a multiplicity-free branching diagram $\widehat{H}$ associated with the tower, namely the branching diagram for the tower $\left(H_{n}^{F}\right)_{n \geq 0}$ of split semisimple algebras over $F$. The edges in the branching diagram are determined as follows: For $\lambda \in \widehat{H}_{n}$ and $\mu \in \widehat{H}_{n+1}, \lambda \rightarrow \mu$ if and only if $\Delta_{H_{n}}^{\lambda}$ appears as a subquotient in a cell filtration of $\operatorname{Res}_{H_{n}}^{H_{n+1}}\left(\Delta_{H_{n+1}}^{\mu}\right)$.

Fix once and for all an order preserving cell filtration of $\operatorname{Res}_{H_{n}}^{H_{n+1}}\left(\Delta_{H_{n+1}}^{\mu}\right)$ for each $n \geq 0$ and each $\mu \in \widehat{H}_{n+1}$ :

$$
\{0\}=M_{0} \stackrel{\lambda^{(1)}}{\subseteq} M_{1} \stackrel{\lambda^{(2)}}{\subseteq} \cdots \stackrel{\lambda^{(r)}}{\subseteq} M_{r}=\operatorname{Res}_{H_{n}}^{H_{n+1}}\left(\Delta_{H_{n+1}}^{\mu}\right) .
$$

Let $\delta_{H_{n+1}}^{\mu}$ be a generator of $\Delta_{H_{n+1}}^{\mu}$. Following observation (1) in Section 3.4, for each edge $\lambda \rightarrow \mu$ in $\widehat{H}$, fix an element $d_{\lambda \rightarrow \mu}^{(n+1)} \in H_{n+1}$ such that $\delta_{H_{n+1}}^{\mu} d_{\lambda^{(j)} \rightarrow \mu}^{(n+1)}+M_{j-1}$ is a generator of $M_{j} / M_{j-1}$. Note that the cell modules of $H_{1}$ have rank 1 , and we can choose all the elements $d_{\emptyset \rightarrow \mu}^{(1)}$ for $\mu \in \widehat{H}_{1}$ to be 1 .

Now fix $n \geq 1$ and $\lambda \in \widehat{H}_{n}$. For each path $\mathfrak{t}=\left(\emptyset=\lambda^{(0)}, \lambda^{(1)}, \ldots, \lambda^{(n)}=\lambda\right) \in \widehat{H}_{n}^{\lambda}$, define

$$
d_{\mathfrak{t}}=d_{\lambda^{(n-1)} \rightarrow \lambda^{(n)}}^{(n)} d_{\lambda^{(n-2) \rightarrow \lambda^{(n-1)}}}^{(n-1)} \cdots d_{\emptyset \rightarrow \lambda^{(1)}}^{(1)} .
$$

Proposition 3.10 Let $n \geq 1$ and let $\mu \in \widehat{H}_{n}$. Consider our chosen cell filtration of $\operatorname{Res}_{H_{n-1}}^{H_{n}}\left(\Delta_{H_{n}}^{\mu}\right)$,

$$
\{0\}=M_{0} \stackrel{\lambda^{(1)}}{\subseteq} M_{1} \stackrel{\lambda^{(2)}}{\subseteq} \cdots \stackrel{\lambda^{(r)}}{\subseteq} M_{r}=\operatorname{Res}_{H_{n-1}}^{H_{n}}\left(\Delta_{H_{n}}^{\mu}\right)
$$


(1) For $1 \leq j \leq r$,

$$
\left\{\delta_{H_{n}}^{\mu} d_{\mathfrak{t}} \mid \mathfrak{t} \in \widehat{H}_{n}^{\mu} \text { and } \mathfrak{t}(n-1) \in\left\{\lambda^{(1)}, \lambda^{(2)}, \ldots, \lambda^{(j)}\right\}\right\}
$$

is a basis of $M_{j}$.

(2) In particular, $\left\{\delta_{H_{n}}^{\mu} d_{\mathfrak{t}} \mid \mathfrak{t} \in \widehat{H}_{n}^{\mu}\right\}$ is a basis of $\Delta_{H_{n}}^{\mu}$.

Proof Evidently, statement (1) implies statement (2). We prove both statements by induction on $n$, the case $n=1$ being evident. Fix $n>1$ and suppose the statements hold for cell modules of $H_{k}$ for $1 \leq k \leq n-1$. For each $i$ we have an isomorphism of $H_{n-1}$-modules

$$
\varphi_{i}: \delta_{H_{n}}^{\mu} d_{\lambda^{(i)} \rightarrow \mu}^{(n)} h+M_{i-1} \mapsto \delta_{H_{n-1}}^{\lambda^{(i)}} h
$$

from $M_{i} / M_{i-1}$ to $\Delta_{H_{n-1}}^{\lambda^{(i)}}$. By the induction hypothesis, $\left\{\delta_{H_{n-1}}^{\lambda^{(i)}} d_{\mathfrak{s}} \mid \mathfrak{s} \in \widehat{H}_{n-1}^{\lambda^{(i)}}\right\}$ is a basis of the cell module $\Delta_{H_{n-1}}^{\lambda^{(i)}}$. Pulling back this basis via $\varphi_{i}$, we get that

$$
\left\{\delta_{H_{n}}^{\mu} d_{\lambda^{(i)} \rightarrow \mu}^{(n)} d_{\mathfrak{s}}+M_{i-1} \mid \mathfrak{s} \in \widehat{H}_{n-1}^{\lambda^{(i)}}\right\}
$$

is a basis of $M_{i} / M_{i-1}$. It follows that for each $j$,

$$
\left\{\delta_{H_{n}}^{\mu} d_{\lambda^{(i)} \rightarrow \mu}^{(n)} d_{\mathfrak{s}} \mid 1 \leq i \leq j \text { and } \mathfrak{s} \in \widehat{H}_{n-1}^{\lambda^{(i)}}\right\}
$$

is a basis of $M_{j}$. But this basis is equal to

$$
\left\{\delta_{H_{n}}^{\mu} d_{\mathfrak{t}} \mid \mathfrak{t} \in \widehat{H}_{n}^{\mu} \text { and } \mathfrak{t}(n-1) \in\left\{\lambda^{(1)}, \lambda^{(2)}, \ldots, \lambda^{(j)}\right\}\right\} .
$$

This proves statement (1), and statement (2) follows.

Corollary 3.11 For each $n$ and $\lambda \in \widehat{H}_{n}$, let $c_{\lambda}$ be a lifting in $H_{n}^{\unrhd \lambda}$ of $\alpha_{\lambda}^{-1}\left(\left(\delta_{H_{n}}^{\lambda}\right)^{*} \otimes \delta_{H_{n}}^{\lambda}\right)$. Then

$$
\left\{d_{\mathfrak{s}}^{*} c_{\lambda} d_{\mathfrak{t}} \mid \lambda \in \widehat{H}_{n} \text { and } \mathfrak{s}, \mathfrak{t} \in \widehat{H}_{n}^{\lambda}\right\}
$$

is a cellular basis of $H_{n}$ which is equivalent to the original cellular basis $\mathscr{H}_{n}$.

Proof Follows from Proposition 3.10 and Lemma 2.10.

Lemma 3.12 The family of bases $\left\{\delta_{H_{n}}^{\lambda} d_{\mathfrak{t}} \mid \mathfrak{t} \in \widehat{H}_{n}^{\lambda}\right\}$ of the cell modules $\Delta_{H_{n}}^{\lambda}$ is a family of path bases.

Proof This is a special case of [14, Proposition 2.18].

\section{Example: The Iwahori-Hecke Algebra of the Symmetric Groups}

In this section, we apply the theory of Section 3 to the Iwahori-Hecke algebra of the symmetric groups. In particular, we recall that the sequence of Hecke algebras is a coherent tower of cyclic cellular algebras, and we compute the branching factors for reduced and induced cell modules. We show that the path bases obtained via ordered products of branching factors coincide with the Murphy bases. 


\subsection{Combinatorics}

Let $n$ denote a non-negative integer and $\mathfrak{S}_{n}$ be the symmetric group acting on $\{1, \ldots, n\}$ on the right. For $i$ an integer, $1 \leq i<n$, let $s_{i}$ denote the transposition $(i, i+1)$. Then $\mathfrak{S}_{n}$ is presented as a Coxeter group by generators $s_{1}, s_{2}, \ldots, s_{n-1}$, with the relations

$$
\begin{array}{ll}
s_{i}^{2}=1, & \text { for } i=1, \ldots, n-1, \\
s_{i} s_{j}=s_{j} s_{i}, & \text { for }|i-j|>1 . \\
s_{i} s_{i+1} s_{i}=s_{i+1} s_{i} s_{i+1}, & \text { for } i=1, \ldots, n-2 .
\end{array}
$$

A product $w=s_{i_{1}} s_{i_{2}} \cdots s_{i_{j}}$ in which $j$ is minimal is called a reduced expression for $w$ and $j=l(w)$ is the length of $w$.

We will assume familiarity with the usual combinatorics associated with the symmetric groups: compositions and partitions, and their diagrams, tableaux, dominance order, etc. We will follow the terminology and notations of [28], especially Section 3.1. Our convention regarding diagrams is illustrated by the example: for the partition $\lambda=(3,2)$, its diagram is $[\lambda]=$\begin{tabular}{l|l|l}
\hline & $\square$
\end{tabular} .

The notation $\lambda \vdash n$ indicates that $\lambda$ is a partition of $n$. The diagram of a partition is commonly called a Young diagram. We denote the set of Young diagrams of size $n$ by $\mathcal{Y}_{n}$ For a composition $\lambda$ of size $n$, let $\mathcal{T}(\lambda)$ denote the set of all $\lambda$-tableaux (possibly with repeated entries) and $\mathcal{T}_{0}(\lambda)$ the set of $\lambda$-tableaux in which each number $1,2, \ldots, n$ appears exactly once. For a partition $\lambda$, write $\mathcal{T}^{\operatorname{Std}}(\lambda)$ for the set of standard $\lambda$-tableaux. If $\mathfrak{t} \in \mathcal{T}_{0}(\lambda)$ and $1 \leq k \leq n$, we write node ${ }_{\mathfrak{t}}(k)$ for the node in $\lambda$ containing the entry $k$, $\operatorname{row}_{\mathfrak{t}}(k)$ for the row coordinate of $k$ in $\mathfrak{t}$ and $\operatorname{col}_{\mathfrak{t}}(k)$ for the column coordinate of $k$ in $\mathfrak{t}$.

The symmetric group $\mathfrak{S}_{n}$ acts freely and transitively on $\mathcal{T}_{0}(\lambda)$, on the right, by acting on the integer labels of the nodes of $[\lambda]$. For example,

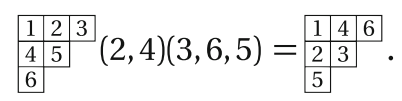

Let $\mathfrak{t}^{\lambda}$ denote the standard $\lambda$-tableau in which $1,2, \ldots, n$ are entered in increasing order from left to right along the rows of $[\lambda]$. Thus in the previous example where $n=6$ and $\lambda=(3,2,1)$,

$$
\mathfrak{t}^{\lambda}=\begin{array}{|l|l|l|}
\hline 1 & 2 & 3 \\
\hline 4 & 5 & \\
\cline { 1 - 1 } 6 &
\end{array} .
$$

For each $\mathfrak{t} \in \mathcal{T}_{0}(\lambda)$, let $w(\mathfrak{t})$ denote the unique permutation such that $\mathfrak{t}=\mathfrak{t}^{\lambda} w(\mathfrak{t})$. The Young subgroup $\mathfrak{S}_{\lambda}$ is defined to be the row stabilizer of $\mathfrak{t}^{\lambda}$ in $\mathfrak{S}_{n}$. For instance, when $n=6$ and $\lambda=(3,2,1)$, as in (4.1), then $\mathfrak{S}_{\lambda}=\left\langle s_{1}, s_{2}, s_{4}\right\rangle$.

Let $\lambda \vdash n$ and let $\mathfrak{t} \in \mathcal{T}_{0}(\lambda)$. Let $\alpha$ be an addable node of $\lambda$. Then we write $\mathfrak{t} \cup \alpha$ for the tableau of shape $\lambda \cup \alpha$ which agrees with $\mathfrak{t}$ on the nodes of $\lambda$ and which has the entry $n+1$ in node $\alpha$. If $\mathfrak{t}$ is a standard $\lambda$-tableau, then the node of $\mathfrak{t}$ containing the entry $n$ is a removable node $\beta$ of $\lambda$. Write $\mathfrak{t}^{\prime}=\mathfrak{t} \downarrow_{n-1}$ for the standard tableau of shape $\lambda \backslash \beta$ obtained by removing the node $\beta$.

\subsection{Iwahori-Hecke Algebras of the Symmetric Group}

Let $R$ be an integral domain and $q$ be a unit in $R$. Let $\mathcal{H}_{n}=\mathcal{H}_{n}\left(q^{2}\right)$ denote the IwahoriHecke algebra of the symmetric group, which is presented by the generators $T_{1}, \ldots, T_{n-1}$, 
and the relations

$$
\begin{array}{ll}
T_{i} T_{j}=T_{j} T_{i}, & \text { if }|i-j|>1, \\
T_{i} T_{i+1} T_{i}=T_{i+1} T_{i} T_{i+1}, & \text { for } i=1, \ldots, n-2, \\
\left(T_{i}-q\right)\left(T_{i}+q^{-1}\right)=0, & \text { for } i=1, \ldots, n-1 .
\end{array}
$$

If we need to refer explicitly to the ground ring $R$, we write $\mathcal{H}_{n}\left(R ; q^{2}\right)$. If $v \in \mathfrak{S}_{n}$, and $v=s_{i_{1}} s_{i_{2}} \cdots s_{i_{l}}$ is a reduced expression for $v$ in $\mathfrak{S}_{n}$, then $T_{v}=T_{i_{1}} T_{i_{2}} \cdots T_{i_{l}}$ is well defined in $\mathcal{H}_{n}\left(q^{2}\right)$ and $\left\{T_{v} \mid v \in \mathfrak{S}_{n}\right\}$ freely generates $\mathcal{H}_{n}\left(q^{2}\right)$ as an $R$-module. It follows from this that $\mathcal{H}_{n}$ embeds in $\mathcal{H}_{n+1}$ for all $n \geq 0$. The $R$-module map $*: T_{v} \mapsto T_{v^{-1}}$ is an algebra anti-automorphism of $\mathcal{H}_{n}\left(q^{2}\right)$. If $i, j=1, \ldots, n$, let

$$
T_{i, j}=\left\{\begin{array}{l}
T_{i} T_{i+1} \cdots T_{j-1}, \text { if } j \geq i \\
T_{i-1} T_{i-2} \cdots T_{j}, \text { if } i>j .
\end{array}\right.
$$

If $R$ is a field of characteristic zero and $q$ is not a proper root of unity, then it is known that each of the algebras $\mathcal{H}_{n}$ is split semisimple with simple modules labeled by the set $\mathcal{Y}_{n}$ of Young diagrams of size $n$; moreover the branching diagram $\widehat{\mathcal{H}}$ of the tower $\left(\mathcal{H}_{n}\right)_{n \geq 0}$ is Young's lattice; namely for Young diagrams $\lambda$ and $\mu$ with $|\mu|=|\lambda|+1$, we have $\lambda \rightarrow \mu$ if and only if $\mu$ is obtained from $\lambda$ by adjoining one node.

If $\mu \in \widehat{\mathcal{H}}_{n}$, define $\widehat{\mathcal{H}}_{n}^{\mu}$ to be the set of paths $\left(\mu^{(0)}=\emptyset, \mu^{(1)}, \ldots, \mu^{(n)}=\mu\right)$ on Young's lattice $\widehat{\mathcal{H}}$ from $\emptyset$ to $\mu$. There is an evident bijection between the set of such paths and standard tableaux of shape $\lambda$.

If $\mu \in \widehat{\mathcal{H}}_{n}$, let

$$
m_{\mu}=\sum_{v \in \mathfrak{S}_{\mu}} q^{l(v)} T_{v} .
$$

In the following statement, recall that for $\lambda \in \widehat{\mathcal{H}}_{i}$ and $\mathfrak{t} \in \mathcal{T}^{\operatorname{Std}}(\lambda), w(\mathfrak{t})$ denotes the unique permutation in $\mathfrak{S}_{i}$ such that $\mathfrak{t}^{\lambda} w(\mathfrak{t})=\mathfrak{t}$.

Theorem 4.1 (Murphy [33]) For $i \geq 1$,

$$
\mathscr{H}_{i}=\left\{m_{\mathfrak{s t}}^{\lambda}=T_{w(\mathfrak{s})}^{*} m_{\lambda} T_{w(\mathfrak{t})} \mid \mathfrak{s}, \mathfrak{t} \in \mathcal{T}^{\mathrm{Std}}(\lambda), \lambda \in \widehat{\mathcal{H}}_{i}\right\}
$$

is an $R$-basis for $\mathcal{H}_{i}$, and $\left(\mathcal{H}_{i}, *, \widehat{\mathcal{H}}_{i}, \unrhd, \mathscr{H}_{i}\right)$ is a cell datum for $\mathcal{H}_{i}$.

Remark 4.2 The basis elements defined here actually differ by a power of $q$ from those defined by Murphy. Murphy and other authors use generators (call them $\tilde{T}_{i}$ ) for $\mathcal{H}_{n}$ satisfying $\left(\tilde{T}_{i}-q^{2}\right)\left(\tilde{T}_{i}+1\right)=0$. These are related to our generators by $\tilde{T}_{i}=q T_{i}$ Thus Murphy's basis elements would be $q^{l(\mathfrak{s})+l(\mathfrak{t})} m_{\mathfrak{s t}}^{\lambda}$.

We let $\left\{m_{\mathfrak{t}}^{\lambda} \mid \mathfrak{t} \in \mathcal{T}^{\text {Std }}(\lambda)\right\}$ denote the basis of the cell module $\Delta_{\mathcal{H}_{n}}^{\lambda}$ derived from the Murphy basis. Then we have $m_{\mathfrak{t}}^{\lambda}=m_{\mathfrak{t}^{\lambda}}^{\lambda} T_{w(\mathfrak{t})}$. In particular, we see that the Hecke algebra is a cyclic cellular algebra, with $\Delta_{\mathcal{H}_{n}}^{\lambda}$ generated by $m_{\mathfrak{t}^{\lambda}}^{\lambda}$. The bimodule isomorphism $\alpha_{\lambda}: \mathcal{H}^{\unrhd \lambda} / \mathcal{H}^{\triangleright \lambda} \rightarrow\left(\Delta_{\mathcal{H}_{n}}^{\lambda}\right)^{*} \otimes \Delta_{\mathcal{H}_{n}}^{\lambda}$ is $\alpha_{\lambda}: m_{\mathfrak{s t}}^{\lambda}+\mathcal{H}^{\triangleright \lambda} \mapsto T_{w(\mathfrak{s})}^{*}\left(m_{\mathfrak{t}^{\lambda}}^{\lambda}\right)^{*} \otimes m_{\mathfrak{t}^{\lambda}}^{\lambda} T_{w(\mathfrak{t})}$. In particular $m_{\lambda}$ is a lift in $\mathcal{H}^{\unrhd \lambda}$ of $\alpha_{\lambda}^{-1}\left(\left(m_{\mathfrak{t}^{\lambda}}^{\lambda}\right)^{*} \otimes m_{\mathfrak{t}^{\lambda}}^{\lambda}\right)$, so plays the role of the element $c_{\lambda}$ in Section 2.5.

We record this as a corollary:

Corollary 4.3 The Hecke algebras $\mathcal{H}_{n}$ are cyclic cellular algebras. 


\subsection{Cell Filtrations and Branching Factors}

Our next task is to recall that the sequence of Hecke algebras $\left(\mathcal{H}_{n}\right)_{n \geq 0}$ is a strongly coherent tower of cellular algebras, and to determine the branching factors $d_{\mu \rightarrow \lambda}^{(n)}$ and $u_{\mu \rightarrow \lambda}^{(n)}$ when $\mu \rightarrow \lambda$. First we discuss the cell filtrations of restrictions of cell modules and the branching factors $d_{\mu \rightarrow \lambda}^{(n)}$.

Theorem 4.4 (Jost, Murphy) Let $n \geq 1$ and $\lambda \in \widehat{\mathcal{H}}_{n}$. Let $\Delta_{\mathcal{H}_{n}}^{\lambda}$ be the corresponding cell module of $\mathcal{H}_{n}$. Then $\operatorname{Res}_{\mathcal{H}_{n-1}}^{\mathcal{H}_{n}}\left(\Delta_{\mathcal{H}_{n}}^{\lambda}\right)$ has an order preserving filtration by cell modules of $\mathcal{H}_{n-1}$.

Jost [22] has shown, using the Dipper-James description of Specht modules of the Hecke algebras [5], that the restriction of a Specht module has a filtration by Specht modules. Together with Murphy's result that the cell modules of the Hecke algebras can be identified with the Specht modules [33, Theorem 5.3], this shows that the restriction of a cell module has a cell filtration. A direct proof of Theorem 4.4 using Murphy's description of the cellular structure is given in [15].

We now give a more precise description of the cell filtration in Theorem 4.4. Let $\alpha_{1}, \ldots, \alpha_{p}$ be the list of removable nodes of $\lambda$, listed from bottom to top and let $\mu^{(j)}=$ $\lambda \backslash \alpha_{j}$. Thus $i \leq j$ if and only if $\mu^{(i)} \unrhd \mu^{(j)}$. Let $N_{0}=(0)$ and for $1 \leq j \leq p$, let $N_{j}$ be the $R$-submodule of $\Delta_{\mathcal{H}_{n}}^{\lambda}$ spanned by by the basis elements $m_{\mathfrak{t}}^{\lambda}$ such that the node containing $n$ in $\mathrm{t}$ is one of $\alpha_{1}, \ldots, \alpha_{j}$. Then we have

$$
(0)=N_{0} \subseteq N_{1} \cdots \subseteq N_{p}=\operatorname{Res}_{\mathcal{H}_{n-1}}^{\mathcal{H}_{n}}\left(\Delta_{\mathcal{H}_{n}}^{\lambda}\right) .
$$

The explicit form of the assertion of Theorem 4.4 is that the $N_{j}$ are $\mathcal{H}_{n-1}$-submodules of $\operatorname{Res}_{\mathcal{H}_{n-1}}^{\mathcal{H}_{n}}\left(\Delta_{\mathcal{H}_{n}}^{\lambda}\right)$ and $N_{j} / N_{j-1} \cong \Delta_{\mathcal{H}_{n-1}}^{\mu^{(j)}}$ for $1 \leq j \leq p$. The isomorphism is determined by

$$
m_{\mathfrak{s}}^{\mu^{(j)}} \mapsto m_{\mathfrak{s} \cup \alpha_{j}}^{\lambda}+N_{j-1}
$$

We can now determine the branching factors $d_{\mu \rightarrow \lambda}^{(n)}$ :

Corollary 4.5 The branching factors $d_{\mu \rightarrow \lambda}^{(n)}$ can be chosen as follows: Let $\lambda \in \widehat{\mathcal{H}}_{n}$ and $\mu \in \widehat{\mathcal{H}}_{n-1}$ with $\mu \rightarrow \lambda$. Let $\alpha=\lambda \backslash \mu$. Then

$$
d_{\mu \rightarrow \lambda}^{(n)}=T_{w\left(\mathfrak{t}^{\mu} \cup \alpha\right)} .
$$

More explicitly, let $a(\alpha)$ be the entry of $\mathfrak{t}^{\lambda}$ in the node $\alpha$. Then $w\left(\mathfrak{t}^{\mu} \cup \alpha\right)=(n, n-$ $1, \ldots, a(\alpha))$, so

$$
d_{\mu \rightarrow \lambda}^{(n)}=T_{(n, n-1, \ldots, a(\alpha))}=T_{a(\alpha), n} .
$$

Proof Under the isomorphism $\Delta_{\mathcal{H}_{n-1}}^{\mu^{(j)}} \rightarrow N_{j} / N_{j-1}$, the generator $m_{\mathfrak{t}^{\mu}(j)}^{\mu^{(j)}}$ is sent to

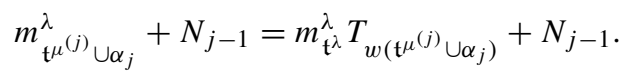

This means that we can chose $d_{\mu \rightarrow \lambda}^{(n)}=T_{w\left(\mathfrak{t}^{\mu} \cup \alpha\right)}$. Now it is straightforward to check that $w\left(\mathfrak{t}^{\mu} \cup \alpha\right)=(n, n-1, \ldots, a(\alpha))$, so that $d_{\mu \rightarrow \lambda}^{(n)}=T_{(n, n-1, \ldots, a(\alpha))}=T_{a(\alpha), n}$. 
Let $\lambda \in \widehat{H}_{n}$ and let $\mathfrak{t}$ be a standard $\lambda$-tableau. We identify $\mathfrak{t}$ with a path on the branching $\operatorname{diagram} \widehat{\mathcal{H}}, \mathfrak{t}=\left(\emptyset=\lambda^{(0)}, \ldots, \lambda^{(n)}=\lambda\right)$. Define

$$
d_{\mathfrak{t}}=d_{\lambda^{(n-1)} \rightarrow \lambda^{(n)}}^{(n)} d_{\lambda^{(n-2)} \rightarrow \lambda^{(n-1)}}^{(n-1)} \cdots d_{\lambda^{(0)} \rightarrow \lambda^{(1)}}^{(1)} .
$$

Lemma 4.6 Let $\lambda$ be a partition of $n$, let $\alpha$ be a removable node of $\lambda$, and let $\mu=\lambda \backslash \alpha$. Let $a(\alpha)$ be the entry of $t^{\lambda}$ in the node $\alpha$. Let $\mathfrak{s} \in \mathcal{T}_{0}(\mu)$ be a $\mu$-tableau. Then

$$
w(\mathfrak{s} \cup \alpha)=(n, n-1, \ldots, a(\alpha)) w(\mathfrak{s}),
$$

and

$$
T_{w(\mathfrak{s} \cup \alpha)}=T_{(n, n-1, \ldots, a(\alpha))} T_{w(\mathfrak{s})}=T_{a(\alpha), n} T_{w(\mathfrak{s})} .
$$

Proof We have

$$
\mathfrak{s} \cup \alpha=\left(\mathfrak{t}^{\mu} \cup \alpha\right) w(\mathfrak{s})=\mathfrak{t}^{\lambda}(n, n-1, \ldots, a(\alpha)) w(\mathfrak{s}) .
$$

Therefore,

$$
w(\mathfrak{s} \cup \alpha)=(n, n-1, \ldots, a(\alpha)) w(\mathfrak{s}) .
$$

Now one can check that $(n, n-1, \ldots, a(\alpha))$ is a distinguished left coset representative of $\mathfrak{S}_{n-1}$ in $\mathfrak{S}_{n}$. Therefore,

$$
T_{w(\mathfrak{s} \cup \alpha)}=T_{(n, n-1, \ldots, a(\alpha))} T_{w(\mathfrak{s})}=T_{a(\alpha), n} T_{w(\mathfrak{s})} .
$$

Lemma 4.7 Let $\lambda$ be a partition of $n$ and let $\mathfrak{t}$ be a standard $\lambda$-tableau. Then $T_{w(\mathfrak{t})}=d_{\mathfrak{t}}$.

Proof Let $\alpha$ be the node of $\lambda$ containing the entry $n$ in $\mathfrak{t}$ and let $\mu=\lambda \backslash \alpha$. Let $\mathfrak{t}^{\prime}$ be the standard $\mu$ tableau obtained from $\mathfrak{t}$ by removing the node $\alpha$. Let $a(\alpha)$ be the entry of $\mathfrak{t}^{\lambda}$ in the node $\alpha$. Then $\mathfrak{t}=\mathfrak{t}^{\prime} \cup \alpha$, so by the previous lemma and Corollary 4.5,

$$
T_{w(\mathfrak{t})}=T_{a(\alpha), n} T_{w\left(\mathfrak{t}^{\prime}\right)}=d_{\mu \rightarrow \lambda}^{(n)} T_{w\left(\mathfrak{t}^{\prime}\right)} .
$$

By induction, we obtain the desired formula $T_{w(\mathfrak{t})}=d_{\mathfrak{t}}$.

Corollary 4.8 The bases of the cell modules and the cellular basis of the Hecke algebra $\mathcal{H}_{n}$ given in Proposition 3.10 and Corollary 3.11 coincide with the Murphy bases:

$$
m_{\mathfrak{t}}^{\lambda}=m_{\mathfrak{t}^{\lambda}}^{\lambda} d_{\mathfrak{t}}, \quad \text { and } \quad m_{\mathfrak{s t}}^{\lambda}=d_{\mathfrak{s}}^{*} m_{\lambda} d_{\mathfrak{t}} .
$$
$u_{\mu \rightarrow v}^{(n)}$.

Next we turn to the cell filtration of induced cell modules and the branching factors

Theorem 4.9 (Dipper-James, Murphy, Mathas) Let $\mu \in \widehat{\mathcal{H}}_{n}$ and let $\Delta_{\mathcal{H}_{n}}^{\mu}$ be the corresponding cell module of $\mathcal{H}_{n}$. Then $\operatorname{Ind}_{\mathcal{H}_{n}}^{\mathcal{H}_{n+1}}\left(\Delta_{\mathcal{H}_{n}}^{\mu}\right)$ has an order preserving filtration by cell modules of $\mathcal{H}_{n+1}$.

Corollary 4.10 The sequence of Hecke algebras $\left(\mathcal{H}_{n}\right)_{n \geq 0}$ is a strongly coherent tower of cyclic cellular algebras.

Proof Combine Theorem 4.4, Theorem 4.9 and Corollary 4.3. 
Let $\alpha=\alpha_{1}, \alpha_{2}, \ldots, \alpha_{p}=\omega$ be the list of addable nodes of $\mu$, listed from top to bottom. Let $v^{(i)}=\mu \cup \alpha_{i}$. Note that $i \leq j$ if and only if $v^{(i)} \unrhd v^{(j)}$. The cell modules of $\mathcal{H}_{n+1}$ occurring as subquotients in the cell filtration of $\operatorname{Ind}_{\mathcal{H}_{n}}^{\mathcal{H}_{n+1}}\left(\Delta_{\mathcal{H}_{n}}^{\mu}\right)$ are $\Delta_{\mathcal{H}_{n+1}}^{v^{(i)}}$ for $1 \leq i \leq p$.

One proof of Theorem 4.9 is obtained by combining [5, Sect. 7] with [33, Theorem 5.3]. A different proof was recently given by Mathas [30]; this proof is based on Murphy's Theorem 4.12 on the existence of a cell filtration of permutation modules of $\mathcal{H}_{n}$. We are going to sketch Mathas' proof in order to point out how the branching factors $u_{\mu \rightarrow v}^{(n+1)}$ can be extracted from it.

Definition 4.11 Let $\lambda, \mu \vdash n$ and $\mathrm{T}: \lambda \rightarrow \mathbb{N}$ be a $\lambda$-tableau. Then:

(1) $\mathrm{T}$ is a tableau of type $\mu$ if for all $i \geq 1, \mu_{i}=\sharp\{a \in \lambda \mid \mathrm{T}(a)=i\}$.

(2) $\mathrm{T}$ is semistandard if the entries of $\mathrm{T}$ are weakly increasing along each row from left to right and strictly increasing along each column from top to bottom.

Let $\mathcal{T}_{\mu}^{\mathrm{SStd}}(\lambda)$ be the set of semistandard $\lambda$-tableaux of type $\mu$ and $\mathcal{T}_{\mu}^{\mathrm{SStd}}\left(\widehat{\mathcal{H}}_{n}\right)=$ $\bigcup_{\lambda \in \widehat{\mathcal{H}}_{n}} \mathcal{T}_{\mu}^{\text {SStd }}(\lambda)$ be the set of all semistandard tableaux of type $\mu$.

Let $\lambda, \mu \vdash n$ and $\mathfrak{t} \in \mathcal{T}^{\text {Std }}(\lambda)$. Define $\mu(\mathfrak{t})$ to be the tableau obtained from $\mathfrak{t}$ by replacing each entry $j$ in $\mathfrak{t}$ with the row index of the entry $j$ in $\mathfrak{t}^{\mu}$. If $\mathcal{T}_{\mu}^{\mathrm{SStd}}(\lambda) \neq \emptyset$, then $\lambda \unrhd \mu$. Note that there is a unique element $\mathrm{T}^{\mu} \in \mathcal{T}_{\mu}^{\mathrm{SStd}}(\mu)$, namely $\mathrm{T}^{\mu}=\mu\left(\mathfrak{t}^{\mu}\right)$.

If $\mathrm{S} \in \mathcal{T}_{\mu}^{\mathrm{SStd}}(\lambda)$ and $\mathfrak{t} \in \mathcal{T}^{\mathrm{Std}}(\lambda)$, let

$$
m_{\mathrm{St}}=\sum_{\substack{\mathfrak{s} \in \mathcal{T}^{\mathrm{Std}}(\lambda) \\ \mu(\mathfrak{s})=\mathrm{S}}} q^{l(w(\mathfrak{s}))} m_{\mathfrak{s t}}^{\lambda}
$$

Let $\mu \in \widehat{\mathcal{H}}_{n}$. Define the permutation module

$$
M^{\mu}=m_{\mu} \mathcal{H}_{n} .
$$

Theorem 4.12 (See [33, Theorem 7.2]) If $\mu \in \widehat{\mathcal{H}}_{n}$, then:

(1) $M^{\mu}$ is free as an $R$-module, with basis

$$
\left\{m_{\mathrm{St}} \mid \mathrm{S} \in \mathcal{T}_{\mu}^{\mathrm{SStd}}(\lambda), \mathfrak{t} \in \mathcal{T}^{\mathrm{Std}}(\lambda) \text { for } \lambda \in \widehat{\mathcal{H}}_{n}\right\} .
$$

(2) Suppose that $\mathcal{T}_{\mu}^{\mathrm{SStd}}\left(\widehat{\mathcal{H}}_{n}\right)=\left\{\mathrm{S}_{1}, \ldots, \mathrm{S}_{k}\right\}$ is ordered so that $i \leq j$ whenever $\lambda^{(i)} \unrhd \lambda^{(j)}$, where $\lambda^{(i)}=\operatorname{Shape}\left(\mathrm{S}_{i}\right)$. Let $M_{i}$ be the $R$-submodule of $M^{\mu}$ spanned by the elements $\left\{m_{\mathrm{S}_{j}} \mathfrak{t} \mid j \leq i\right.$ and $\left.\mathfrak{t} \in \mathcal{T}^{\operatorname{Std}}\left(\lambda^{(j)}\right)\right\}$. Then

$$
\{0\}=M_{0} \stackrel{\lambda^{(1)}}{\subseteq} M_{1} \stackrel{\lambda^{(2)}}{\subseteq} \cdots \stackrel{\lambda^{(m)}}{\subseteq} M_{m}=M^{\mu}
$$

is a cell module filtration of $M^{\mu}$. The isomorphism $M_{j} / M_{j-1} \cong \Delta_{\mathcal{H}_{n}}^{\lambda^{(j)}}$ is determined by

$$
m_{\mathrm{S}_{j} \mathfrak{t}}+M_{j-1} \mapsto m_{\mathfrak{t}}^{\lambda^{(j)}}, \quad \text { for } \mathfrak{t} \in \mathcal{T}^{\mathrm{Std}}\left(\lambda^{(j)}\right) .
$$

Remark 4.13 In Theorem 4.12, we have $\mathrm{S}_{m}=\mathrm{T}^{\mu}$ and $\lambda^{(m)}=\mu$.

Since $\mathcal{H}_{n+1}$ is free of rank $n+1$ as a left $\mathcal{H}_{n}$-module, it follows that the induction functor $\operatorname{Ind}_{\mathcal{H}_{n}}^{\mathcal{H}_{n+1}}\left(\_\right)=\_\otimes_{\mathcal{H}_{n}} \mathcal{H}_{n+1}$ is exact. We will write Ind for this functor in the following 
discussion. Because of exactness, we have

$$
\operatorname{Ind}\left(M_{j}\right) / \operatorname{Ind}\left(M_{j-1}\right) \cong \operatorname{Ind}\left(M_{j} / M_{j-1}\right) \cong \operatorname{Ind}\left(\Delta_{\mathcal{H}_{n}}^{\lambda^{(j)}}\right) .
$$

In particular

$$
\operatorname{Ind}\left(M^{\mu}\right) / \operatorname{Ind}\left(M_{m-1}\right) \cong \operatorname{Ind}\left(\Delta_{\mathcal{H}_{n}}^{\mu}\right) .
$$

Mathas' proof of Theorem 4.9 proceeds by exhibiting a cell filtration of $\operatorname{Ind}\left(M^{\mu}\right) / \operatorname{Ind}\left(M_{m-1}\right)$.

Another consequence of the freeness of $\mathcal{H}_{n+1}$ as a left $\mathcal{H}_{n}$-module is the following: if $M$ is a right ideal in $\mathcal{H}_{n}$, then

$$
\operatorname{Ind}(M)=M \otimes_{\mathcal{H}_{n}} \mathcal{H}_{n+1} \cong M \mathcal{H}_{n+1},
$$

via $x \otimes h \mapsto x h$. We will simply identify $\operatorname{Ind}(M)$ with $M \mathcal{H}_{n+1}$. Recall that $\omega$ denotes the lowest addable node of $\mu$, and note that $m_{\mu}=m_{\mu \cup \omega}$. Hence,

$$
\operatorname{Ind}\left(M^{\mu}\right)=M^{\mu} \mathcal{H}_{n+1}=m_{\mu} \mathcal{H}_{n+1}=m_{\mu \cup \omega} \mathcal{H}_{n+1}=M^{\mu \cup \omega} .
$$

To proceed, we need to relate semistandard tableaux of size $n$ and type $\mu$ and semistandard tableaux of size $n+1$ and type $\mu \cup \omega$. Let $l$ denote the number of non-zero parts of $\mu$, so that $\omega=(l+1,1)$. If $\mathrm{S}$ is a semistandard tableau of shape $\lambda$ and type $\mu$, and $\beta$ is an addable node of $\lambda$, then we define the semistandard tableau $S \cup \beta$ of shape $\lambda \cup \beta$ and type $\mu \cup \omega$ by $S \cup \beta(x)=S(x)$ if $x \in[\lambda]$ and $S(\beta)=l+1$. We write $\mathcal{T}_{\mu \cup \omega}^{\mathrm{SStd}}(S)$ for the set of semistandard tableaux $S \cup \beta$ as $\beta$ ranges over addable nodes of $\lambda$. It is easy to see that every $\mathrm{U} \in \mathcal{T}_{\mu \cup \omega}^{\mathrm{SStd}}\left(\widehat{\mathcal{H}}_{n+1}\right)$ is obtained as $\mathrm{S} \cup \beta$ for some $\mathrm{S}$ and some $\beta$.

Recall that $S_{1}, \ldots, S_{m}=T^{\mu}$ is the list of all semistandard tableaux of size $n$ and type $\mu$, listed so that $\operatorname{Shape}\left(S_{i}\right) \unrhd \operatorname{Shape}\left(S_{j}\right)$ implies $i \leq j$. Mathas defines the following $R-$ submodules of $M^{\mu \cup \omega}$ :

$$
N_{i}=\operatorname{span}\left\{m_{\cup \mathfrak{v}} \mid \cup \in \mathcal{T}_{\mu \cup \omega}^{\mathrm{SStd}}\left(S_{j}\right), \mathfrak{v} \in \mathcal{T}^{\mathrm{Std}}(\operatorname{Shape}(\mathrm{U})) \text { for } 1 \leq j \leq i\right\}
$$

Lemma 4.14 ([30, Lemma 3.5]) Let $\mathrm{S} \in \mathcal{T}_{\mu}^{\mathrm{SStd}}(\lambda), U \in \mathcal{T}_{\mu \cup \omega}^{\mathrm{SStd}}(\mathrm{S})$, and $v=\operatorname{Shape}(\mathrm{U})$. Then $m_{\cup^{\nu}} \in m_{\mathrm{St}^{\lambda}} \mathcal{H}_{n+1}$.

Proposition 4.15 ([30, Theorem 3.6]) $N_{m-1}=\operatorname{Ind}\left(M_{m-1}\right)$.

Remark 4.16 The proof of this result in the published version of [30] has a gap, but this was repaired in the version posted to the arxiv.

By applying Theorem 4.12 to $M^{\mu \cup \omega}$, we see that $M^{\mu \cup \omega} / N_{m-1}$ is free with basis

$$
\begin{aligned}
& \left\{m_{\cup \mathfrak{v}}+N_{m-1} \mid \cup \in \mathcal{T}_{\mu \cup \omega}^{\mathrm{SStd}}\left(\mathrm{T}^{\mu}\right), \mathfrak{v} \in \mathcal{T}^{\mathrm{Std}}(\operatorname{Shape}(\mathrm{U}))\right\} \\
& =\left\{m_{\mathrm{T}^{\mu} \cup \beta, \mathfrak{v}}+N_{m-1} \mid \beta \text { is an addable node of } \mu, \mathfrak{v} \in \mathcal{T}^{\text {Std }}(\mu \cup \beta)\right\} .
\end{aligned}
$$

We can now exhibit an order preserving cell filtration of $M^{\mu \cup \omega} / N_{m-1} \cong \operatorname{Ind}\left(\Delta_{\mathcal{H}_{n}}^{\mu}\right)$. In the following, we write $N=N_{m-1}$. Recall that $\alpha=\alpha_{1}, \alpha_{2}, \ldots, \alpha_{p}=\omega$ is the list of addable nodes of $\mu$ listed from top to bottom and $v^{(j)}=\mu \cup \alpha_{j}$. Let $J^{0}=(0)$ and for $1 \leq i \leq p$, define $J^{i} \subseteq M^{\mu \cup \omega} / N$ by

$$
J^{i}=\operatorname{span}\left\{m_{\top^{\mu} \cup \alpha_{j}, \mathfrak{v}}+N \mid j \leq i \text { and } \mathfrak{v} \in \mathcal{T}^{\mathrm{Std}}\left(\mu \cup \alpha_{j}\right)\right\} .
$$


Theorem 4.17 ([30, Corollary 3.7]) Each $J^{i}$ is an $\mathcal{H}_{n+1}$ submodule of $M^{\mu \cup \omega} / N$,

$$
(0)=J^{0} \subseteq J^{1} \subseteq \cdots \subseteq J^{p}=M^{\mu \cup \omega} / N,
$$

and $J^{i} / J^{i-1} \cong \Delta_{\mathcal{H}_{n+1}}^{v^{(i)}}$.

This completes the sketch of Mathas' proof of Theorem 4.9. It remains to see how the cell filtration (4.17) carries over to $\operatorname{Ind}\left(\Delta_{\mathcal{H}_{n}}^{\mu}\right)$, and to identify the branching factors $u_{\mu \rightarrow \nu}^{(n+1)}$. The isomorphism $\varphi: M^{\mu \cup \omega} / N \rightarrow \operatorname{Ind}\left(\Delta_{\mathcal{H}_{n}}^{\mu}\right)$ is the composite of the isomorphism $M^{\mu \cup \omega} / N \cong$ Ind $\left(M^{\mu} / M_{m-1}\right)$, given by

$$
m_{\mu} h+N \mapsto\left(m_{\mu}+M_{m-1}\right) \otimes h,
$$

and the isomorphism $\operatorname{Ind}\left(M^{\mu} / M_{m-1}\right) \cong \operatorname{Ind}\left(\Delta_{\mathcal{H}_{n}}^{\mu}\right)$ given by

$$
\left(m_{\top^{\mu} \mathfrak{t}^{\mu}}+M_{m-1}\right) \otimes h \mapsto m_{\mathfrak{t}^{\mu}}^{\mu} \otimes h .
$$

Since $m_{\top^{\mu} t^{\mu}}=m_{\mu}$, the composite isomorphism is given by

$$
\varphi: m_{\mu} h+N \mapsto m_{\mathfrak{t}^{\mu}}^{\mu} \otimes h .
$$

We need to examine how this isomorphism acts on the basis (4.16) of $M^{\mu \cup \omega} / N$.

Let $\beta$ be an addable node of $\mu$ and let $v=\mu \cup \beta$. Suppose that $\beta$ is in row $r$, and let $a=\sum_{j=1}^{r} v_{j}=1+\sum_{j=1}^{r} \mu_{j}$. Recall that $T_{i, i}=1$ and if $i>j$, then $T_{i, j}=T_{(j, j+1, \ldots, i)}=$ $T_{i-1} T_{i-2} \cdots T_{j}$. Define

$$
\begin{aligned}
D(\beta) & =\sum_{k=0}^{\mu_{r}} q^{k} T_{a, a-k} \\
& =1+q T_{a-1}+q^{2} T_{a-1} T_{a-2}+\cdots+q^{\mu_{r}} T_{a-1} T_{a-2} \cdots T_{a-\mu_{r}} .
\end{aligned}
$$

In particular, $D(\omega)=1$.

The following lemma can be extracted from [30].

\section{Lemma 4.18}

(1) $m_{v}=T_{n+1, a}^{-1} m_{\mu} T_{n+1, a} D(\beta)$.

(2) $w\left(\mathfrak{t}^{\mu} \cup \beta\right)=(n+1, n, \ldots, a)$. Thus $m_{\mathfrak{t}^{v}, \mathfrak{t}^{\mu} \cup \beta}^{v}=m_{v}\left(T_{n+1, a}\right)^{*}$.

(3) $m_{\top^{\mu} \cup \beta, t^{\nu}}=q^{n+1-a} m_{\mu} T_{n+1, a} D(\beta)$.

(4) The isomorphism $\varphi: M^{\mu \cup \omega} / N \rightarrow \operatorname{Ind}\left(\Delta_{\mathcal{H}_{n}}^{\mu}\right)$ satisfies

$$
\varphi\left(m_{\top^{\mu} \cup \beta, \mathfrak{t}^{v}}+N\right)=m_{\mathfrak{t}^{\mu}}^{\mu} \otimes q^{n+1-a} T_{n+1, a} D(\beta) .
$$

Proof If $\beta=\omega$, then $T_{n+1, a}=D(\beta)=1$, and all the statements are evident. Suppose that $\beta \neq \omega$. Let $v^{\prime}$ be the composition $v^{\prime}=\left(\mu_{1}, \ldots, \mu_{r}, 1, \mu_{r+1}, \ldots, \mu_{l}\right)$. One has $T_{n+1, a}^{-1} T_{j} T_{n+1, a}=T_{j+1}$ if $a \leq j \leq n-1$. This follows from the identity in the braid group:

$$
\left(\sigma_{a}^{-1} \cdots \sigma_{n}^{-1}\right) \sigma_{j}\left(\sigma_{n} \cdots \sigma_{a}\right)=\sigma_{j+1}
$$

for $a \leq j \leq n-1$, where the elements $\sigma_{i}$ are the Artin generators of the braid group. From this, we obtain:

$$
m_{v^{\prime}}=T_{n+1, a}^{-1} m_{\mu} T_{n+1, a} .
$$

Note that $\mathfrak{S}_{v^{\prime}} \subset \mathfrak{S}_{v}$ and $D(\beta)=\sum q^{l(x)} T_{x}$, as where the sum is over the distinguished right coset representatives of $\mathfrak{S}_{v^{\prime}}$ in $\mathfrak{S}_{v}$. Hence $m_{v}=m_{v^{\prime}} D(\beta)$, and part (1) follows. The 
first assertion in part (2) is evident and the second statement follows because $T_{(n+1, \ldots, a)}=$ $T_{a, n+1}=\left(T_{n+1, a}\right)^{*}$.

For part (3), $m_{\top^{\mu} \cup \beta, \mathfrak{t}^{\nu}}=\sum_{\mathfrak{s}} q^{l(s)}\left(T_{d(\mathfrak{s})}\right)^{*} m_{\nu}$, where the sum is over standard tableaux $\mathfrak{s}$ of shape $v$ such that $(\mu \cup \omega)(\mathfrak{s})=\mathrm{T}^{\mu} \cup \beta$, according to the definition (4.8). But there is only one such standard tableau, namely $\mathfrak{s}=\mathfrak{t}^{\mu} \cup \beta$. Applying parts (1) and (2),

$$
\begin{aligned}
m_{\mathrm{\top}^{\mu} \cup \beta, \mathfrak{t}^{\nu}} & =q^{n+1-a} T_{n+1, a} m_{v} \\
& =q^{n+1-a} m_{\mu} T_{n+1, a} D(\beta) .
\end{aligned}
$$

Part (4) follows from part (3) together with the description of $\varphi$ in Eq. (4.18).

Corollary 4.19 The branching factors $u_{\mu \rightarrow \nu}^{(n+1)}$ can be chosen as follows: Let $\mu \in \widehat{\mathcal{H}}_{n}$ and $v \in \widehat{\mathcal{H}}_{n+1}$ with $\mu \rightarrow v$. Let $\beta=v \backslash \mu$. Suppose that $\beta$ is in row $r$ and let $a=\sum_{j=1}^{r} v_{j}$. Then:

$$
u_{\mu \rightarrow \nu}^{(n+1)}=T_{n+1, a} D(\beta)=T_{n+1, a} \sum_{k=0}^{\mu_{r}} q^{k} T_{a, a-k}=\sum_{k=0}^{\mu_{r}} q^{k} T_{n+1, a-k}
$$

Proof In Theorem 4.7, we have for $j \geq 1$,

$$
J^{j}=\left(m_{\mathrm{T}^{\mu} \cup \alpha_{j}, \mathrm{t}^{(j)}}+N\right) \mathcal{H}_{n+1}+J^{j-1} .
$$

Set $I^{j}=\varphi\left(J^{j}\right)$. Then $I^{j} / I^{j-1} \cong \Delta_{\mathcal{H}_{n+1}}^{v^{(j)}}$ and

$$
I^{j}=\varphi\left(m_{\mathrm{T}^{\mu} \cup \alpha_{j}, \mathrm{t}^{(j)}}+N\right) \mathcal{H}_{n+1}+I^{j-1} .
$$

Hence, the statement follows from Lemma 4.18, part (4).

\section{Algebras with Jones Basic Construction}

\subsection{Cellularity and the Jones Basic Construction: A Correction}

In [13, 14], Goodman and Graber developed a theory of cellularity for algebras with a Jones basic construction. Examples of such algebras include the Birman-Murakami-Wenzl, Brauer, partition, and Jones-Temperley-Lieb algebras, among others. There was, however, a mistake in the proof in [13] that these algebras constitute coherent towers of cellular algebras. In this section, we will review the setting of [13, 14], describe the error, and explain what needs to be done to correct it.

The setting in [13], as modified in [14] is the following. First recall that an essential idempotent in an algebra $A$ over a ring $R$ is an element $e$ such that $e^{2}=\delta e$ for some nonzero $\delta \in R$. Let $R$ be an integral domain with field of fractions $F$ and consider two towers of algebras with common multiplicative identity,

$$
A_{0} \subseteq A_{1} \subseteq A_{2} \subseteq \cdots \quad \text { and } \quad H_{0} \subseteq H_{1} \subseteq H_{2} \subseteq \cdots .
$$

It is assumed that the two towers satisfy the following list of axioms:

(1) There is an algebra involution $*$ on $\cup_{n} A_{n}$ such that $\left(A_{n}\right)^{*}=A_{n}$, and likewise, there is an algebra involution $*$ on $\cup_{n} H_{n}$ such that $\left(H_{n}\right)^{*}=H_{n}$.

(2) $A_{0}=H_{0}=R$ and $A_{1}=H_{1}$ (as algebras with involution). 
(3) For $n \geq 2, A_{n}$ contains an essential idempotent $e_{n-1}$ such that $e_{n-1}^{*}=e_{n-1}$ and $A_{n} /\left(A_{n} e_{n-1} A_{n}\right) \cong H_{n}$ as algebras with involution.

(4) For $n \geq 1, e_{n}$ commutes with $A_{n-1}$ and $e_{n} A_{n} e_{n} \subseteq A_{n-1} e_{n}$.

(5) For $n \geq 1, A_{n+1} e_{n}=A_{n} e_{n}$, and the map $x \mapsto x e_{n}$ is injective from $A_{n}$ to $A_{n} e_{n}$.

(6) For $n \geq 2, e_{n-1} \in A_{n+1} e_{n} A_{n+1}$.

(7) For all $n, A_{n}^{F}:=A_{n} \otimes_{R} F$ is split semisimple.

(8) $\left(H_{n}\right)_{n \geq 0}$ is a strongly coherent tower of cellular algebras.

Under these hypotheses, it is claimed in $[13,14]$ that $\left(A_{n}\right)_{n \geq 0}$ is a strongly coherent tower of cellular algebras. The strategy of the proof is to show by induction that the following statements hold for all $n \geq 0$ :

- $A_{n}$ is a cellular algebra.

- For $2 \leq n, J_{n}=A_{n} e_{n-1} A_{n}$ is a cellular ideal in $A_{n}$.

- For $2 \leq n$, the cell modules of $J_{n}$ are of the form $\Delta=\Delta^{\prime} \otimes_{A_{n-2}} e_{n-1} A_{n}$, where $\Delta^{\prime}$ is a cell module of $A_{n-2}$.

- The finite tower $\left(A_{k}\right)_{0 \leq k \leq n}$ is strongly coherent.

For $n \leq 1$ these statements are evident. Assuming the statements hold for some fixed $n \geq 1$, one first proves that $J_{n+1}$ is a cellular ideal in $A_{n+1}$ with cell modules of the form $\Delta=$ $\Delta^{\prime} \otimes_{A_{n-1}} e_{n} A_{n+1}$, where $\Delta^{\prime}$ is a cell module of $A_{n-1}$. It follows from Lemma 2.5 that $A_{n+1}$ is cellular.

It then remains to show that for each cell module $\Delta$ of $A_{n+1}$, the restriction of $\Delta$ to $A_{n}$ has an order preserving cell filtration, and that for each cell module $\Delta$ of $A_{n}$, the induction of $\Delta$ to $A_{n+1}$ has an order preserving cell filtration. In fact, we will go over the details of the proof of these last two steps below in Theorem 5.6. For now, we note that in the proof of the statement about induced modules, it was falsely claimed in [13], in the last paragraph on page 335, that if $\Delta$ is a cell module of $J_{n}$ then $\Delta J_{n}=\Delta$. In fact, this does not follow from the axioms (1)-(8) listed above, so it is necessary to add an additional axiom to our framework, as follows:

(9) For $n \geq 2, e_{n-1} A_{n} e_{n-1} A_{n}=e_{n-1} A_{n}$.

From this, it follows that for a cell module $\Delta=\Delta^{\prime} \otimes_{A_{n-2}} e_{n-1} A_{n}$ of $J_{n}$, we have $\Delta J_{n}=\Delta$, and the proof in [13] can proceed as before.

Let us now consider the applicability of the augmented framework axioms (1)-(9) to the principal examples considered in $[13,14]$. In fact, in each example, a stronger version of axiom (6) holds, namely

$$
e_{n-1} e_{n} e_{n-1}=e_{n-1} \quad \text { and } e_{n} e_{n-1} e_{n}=e_{n} \text { for } n \geq 2 .
$$

Thus for $n \geq 3$,

$$
e_{n-1} A_{n} e_{n-1} A_{n} \supseteq e_{n-1} e_{n-2} e_{n_{1}} A_{n}=e_{n-1} A_{n} .
$$

Therefore, Axiom (6) reduces to the statement

$$
e_{1} A_{2} e_{1} A_{2}=e_{1} A_{2} \text {. }
$$

When $A_{n}$ is the $n$-th BMW, Brauer, partition, or Jones-Temperley-Lieb algebra defined over an integral ground ring $R$, we have $A_{1}=H_{1}=R$. Let $\delta$ be the non-zero element of $R$ such that $e_{1}^{2}=\delta e_{1}$. Then we have

$$
e_{1} A_{2} e_{1} A_{2}=e_{1} A_{1} e_{1} A_{2}=e_{1}^{2} A_{2}=\delta e_{1} A_{2},
$$

where we have used $e_{1} A_{2}=e_{1} A_{1}=R e_{1}$. In each of these examples, $e_{1} A_{2}$ is free as an $R-$ module, and hence Axiom (9) holds if and only if $\delta$ is invertible in $R$. It follows that Axiom 
(9) does not hold when $R$ is the generic ground ring, but it does hold when $R$ is the generic ground ring with $\delta^{-1}$ adjoined.

In fact, for these algebras, it is false that $\left(A_{n}\right)_{n \geq 0}$ is a coherent tower of cellular algebras, over the generic ground ring, but, by [13], as corrected above, it is true over the generic ground ring with $\delta^{-1}$ adjoined. This is illustrated by the example of the Jones-TemperleyLieb algebras in the following section.

\subsection{An example: The Jones-Temperley-Lieb Algebras}

We first state an elementary result about the commutativity of specialization and induction.

Let $A$ be an algebra over an integral domain $R$ and let $\varphi: R \rightarrow k$ be a ring homomorphism from $R$ to a field $k$. Write $A^{k}$ for $A \otimes_{R} k$, and for a right $A$-module $M$, write $M^{k}$ for the right $A^{k}$-module $M \otimes_{R} k$.

Lemma 5.1 Let $A \subseteq B$ be algebras over an integral domain $R$, let $\varphi: R \rightarrow k$ be a ring homomorphism from $R$ to a field $k$, and let $M$ be a right $A$-module. Then

$$
\operatorname{Ind}_{A}^{B}(M) \otimes_{R} k \cong \operatorname{Ind}_{A^{k}}^{B^{k}}\left(M^{k}\right),
$$

as right $B^{k}-$ modules.

Corollary 5.2 If, in the situation of the lemma, $\operatorname{Ind}_{A}^{B}(M)$ is free as an $R$-module, then $\operatorname{dim}_{k}\left(\operatorname{Ind}_{A^{k}}^{B^{k}}\left(M^{k}\right)\right)$ is independent of the choice of $k$ and of the homomorphism $\varphi: R \rightarrow k$.

Now we consider the Jones-Temperley-Lieb algebras $A_{n}=A_{n}\left(R_{0} ; \boldsymbol{\delta}\right)$ defined over the generic ground ring $R_{0}=\mathbb{Z}[\delta]$, where $\delta$ is an indeterminant. For the definition of these algebras and a description of their cellular structure, see Section 6.4 of this paper, and further references there.

The algebra $A_{2}$ has two cell modules, each of rank 1. They are $\Delta_{0}=e_{1} A_{2}=R e_{1}$ and $\Delta_{1}=A_{2} / \operatorname{Re}_{1}$. When $k=\mathbb{Q}(\boldsymbol{\delta})$, Ind $\operatorname{IA}_{3}^{A^{k}}\left(\Delta_{0}^{k}\right)$ is two dimensional and $\operatorname{Ind}_{A_{2}^{k}}^{A^{k}}\left(\Delta_{1}^{k}\right)$ is three dimensional, as one sees by examining the generic branching diagram for the tower $\left(A_{n}^{k}\right)_{n \geq 0}$. However, when $k=\mathbb{Q}$ and $\delta=0, \Delta_{0}^{k} \cong \Delta_{1}^{k}$, so also $\operatorname{Ind}_{A_{2}^{k}}^{A_{k}^{k}}\left(\Delta_{0}^{k}\right) \cong \operatorname{Ind}_{A_{2}^{k}}^{A_{k}^{k}}\left(\Delta_{1}^{k}\right)$. It follows from this and Corollary 5.2 that at least one of $\operatorname{Ind}\left(\Delta_{0}\right)$ or $\operatorname{Ind}\left(\Delta_{1}\right)$ fails to be free as an $R$-module, and in particular one of these induced modules does not have a cell filtration.

Corollary 5.3 The tower of Jones-Temperley-Lieb algebras $\left(A_{n}\left(R_{0} ; \delta\right)\right)_{n \geq 0}$ over the generic ground ring $R_{0}=\mathbb{Z}[\delta]$ is not a coherent tower of cellular algebras.

\subsection{Standing Assumptions}

For the remainder of the paper we will work in the setting described by axioms (1)-(9) of Section 5.1, and assume in addition that

(10) Each $H_{n}$ is a cyclic cellular algebra.

\subsection{Cellularity of the Algebras $A_{n}$}

Next we review some of the consequence of our axioms that were obtained in $[13,14]$, as corrected above in Section 5.1. In the following let $\left(H_{i}, *, \widehat{H}_{i}, \unrhd, \mathscr{H}_{i}\right)$ denote the cell datum for $H_{i}$. 
(1) Each $A_{n}$ is a cellular algebra. In fact, this is demonstrated by showing that $J_{n}=$ $A_{n} e_{n-1} A_{n}$ is a cellular ideal of $A_{n}$. Since the quotient algebra $H_{n}=A_{n} / J_{n}$ is assumed to be cellular, it follows from Lemma 2.5 that $A_{n}$ is cellular.

(2) The partially ordered set $\widehat{A}_{n}$ in the cell datum for $A_{n}$ can be realized as

$$
\widehat{A}_{n}=\left\{(\lambda, l) \mid 0 \leq l \leq\lfloor n / 2\rfloor \text { and } \lambda \in \widehat{H}_{n-2 l}\right\},
$$

with the partial order $(\lambda, l) \triangleright(\mu, m)$ if $l>m$ or if $l=m$ and $\lambda \triangleright \mu$ in $\widehat{H}_{n-2 l}$.

(3) The cell modules $\Delta_{A_{n}}^{(\lambda, 0)}$ for $\lambda \in \widehat{H}_{n}$ are those such that $\Delta_{A_{n}}^{(\lambda, 0)} J_{n}=0$. Let $\pi_{n}: A_{n} \rightarrow$ $A_{n} / J_{n}=H_{n}$ denote the quotient map. The cell module $\Delta_{A_{n}}^{(\lambda, 0)}$ can be identified with $\Delta_{H_{n}}^{\lambda}$ via $x a=x \pi_{n}(a)$ for $x \in \Delta_{H_{n}}^{\lambda}$ and $a \in A_{n}$, as in Remark 2.6. The cell modules $\Delta_{A_{n}}^{(\lambda, l)}$ for $l>0$ are the cell modules of the cellular ideal $J_{n}$. For $l>0$, we have

$$
\Delta_{A_{n}}^{(\lambda, l)} \cong \Delta_{A_{n-2}}^{(\lambda, l-1)} \otimes_{A_{n-2}} e_{n-1} A_{n}=\Delta_{A_{n-2}}^{(\lambda, l-1)} \otimes_{A_{n-2}} e_{n-1} A_{n-1} .
$$

(4) The sequence $\left(A_{n}\right)_{n \geq 0}$ is a strongly coherent tower of cellular algebras. Since $A_{n}^{F}$ and $H_{n}^{F}$ are split semisimple for all $n$, the two towers have branching diagrams, by Corollary 3.5.

(5) The branching diagram $\widehat{A}$ for the tower $\left(A_{n}\right)_{n \geq 0}$ is that "obtained by reflections" from the branching diagram $\widehat{H}$ of the tower $\left(H_{n}\right)_{n \geq 0}$. That is, for $(\lambda, l) \in \widehat{A}_{n}$ and $(\mu, m) \in$ $\widehat{A}_{n+1}$, we have $(\lambda, l) \rightarrow(\mu, m)$ only if $m \in\{l, l+1\}$; moreover, $(\lambda, l) \rightarrow(\mu, l)$ if and only if $\lambda \rightarrow \mu$ in $\widehat{H}$, and $(\lambda, l) \rightarrow(\mu, l+1)$ if and only if $\mu \rightarrow \lambda$ in $\widehat{H}$.

Remark 5.4 The parameterization of $\widehat{A}_{n}$ given here differs from that used in [13, 14].

Taking Axiom (10) into account, we obtain:

Theorem 5.5 The tower $\left(A_{n}\right)_{n \geq 0}$ is a strongly coherent tower of cyclic cellular algebras.

Proof From [13, 14], with the correction noted in Section 5.1, we have that the tower is a strongly coherent tower of cellular algebras. It remains to show that each $A_{n}$ is cyclic cellular. We prove this by induction on $n$. The statement is known for $n=0$ and $n=1$, since $A_{0}=R$ and $A_{1}=H_{1}$. Fix $n \geq 0$ and assume the algebras $A_{k}$ for $k \leq n$ are cyclic cellular. The cell modules $\Delta_{A_{n+1}}^{(\lambda, 0)}$ are cell modules of $H_{n+1}$, so cyclic by axiom (9). For $l>0$, we can take

$$
\Delta_{A_{n+1}}^{(\lambda, l)}=\Delta_{A_{n-1}}^{(\lambda, l-1)} \otimes_{A_{n-1}} e_{n} A_{n+1},
$$

By the induction hypothesis, $\Delta_{A_{n-1}}^{(\lambda, l-1)}$ is cyclic, say with generator $\delta_{A_{n-1}}^{(\lambda, l-1)}$. It follows that $\Delta_{A_{n+1}}^{(\lambda, l)}$ is cyclic with generator $\delta_{A_{n+1}}^{(\lambda, l)}=\delta_{A_{n-1}}^{(\lambda, l-1)} \otimes_{A_{n-1}} e_{n}$.

\subsection{Data Associated with the Cell Modules $\Delta_{A_{n}}^{(\lambda, l)}$}

We suppose that generators $\delta_{H_{n}}^{\lambda}$ of $\Delta_{H_{n}}^{\lambda}$ have been chosen for all $n \geq 0$ and for all $\lambda \in \widehat{H}_{n}$. We suppose also that $H_{n}-H_{n}$ bimodule isomorphisms $\alpha_{\lambda}: H_{n}^{\unrhd \lambda} / H_{n}^{\triangleright \lambda} \rightarrow\left(\Delta_{H_{n}}^{\lambda}\right)^{*} \otimes_{R} \Delta_{H_{n}}^{\lambda}$ have been chosen, satisfying $* \circ \alpha_{\lambda}=\alpha_{\lambda} \circ *$. Finally, we suppose that elements $c_{\lambda} \in H_{n}^{\unrhd \lambda}$ have been chosen with $\alpha_{\lambda}\left(c_{\lambda}+H_{n}^{\triangleright \lambda}\right)=\left(\delta_{H_{n}}^{\lambda}\right)^{*} \otimes \delta_{H_{n}}^{\lambda}$.

Now we want to do the following:

(1) establish models of cell modules $\Delta_{A_{n}}^{(\lambda, l)}$ of $A_{n}$ for all $n$ and all $(\lambda, l) \in \widehat{A}_{n}$; 
(2) select generators $\delta_{A_{n}}^{(\lambda, l)}$ for each cell module;

(3) choose $A_{n}-A_{n}$ bimodule isomorphisms

$$
\alpha_{(\lambda, l)}: A^{\unrhd(\lambda, l)} / A^{\triangleright(\lambda, l)} \rightarrow\left(\Delta_{A_{n}}^{(\lambda, l)}\right)^{*} \otimes_{R} \Delta_{A_{n}}^{(\lambda, l)}
$$

satisfying $* \circ \alpha_{(\lambda, l)}=\alpha_{(\lambda, l)} \circ *$;

(4) and finally choose elements $c_{(\lambda, l)} \in A^{\unrhd(\lambda, l)}$ such that

$$
\alpha_{(\lambda, l)}\left(c_{(\lambda, l)}+A^{\triangleright(\lambda, l)}\right)=\left(\delta_{A_{n}}^{(\lambda, l)}\right)^{*} \otimes \delta_{A_{n}}^{(\lambda, l)} .
$$

When $l=0$, we identify $\Delta_{A_{n}}^{(\lambda, 0)}$ with $\Delta_{H_{n}}^{\lambda}$, and we proceed according to the prescription of Remark 2.6 and Remark 2.11. Namely, $\delta_{A_{n}}^{(\lambda, 0)}=\delta_{H_{n}}^{\lambda} ; \alpha_{(\lambda, 0)}: a+A_{n}^{\triangleright(\lambda, 0)} \mapsto \alpha_{\lambda}\left(\pi_{n}(a)+\right.$ $\left.H_{n}^{\triangleright \lambda}\right)$; and $c_{(\lambda, 0)}$ is any element of $\pi_{n}^{-1}\left(c_{\lambda}\right)$.

We continue by induction on $n$. For $n \leq 1$ there is nothing to do, since $A_{0}=R$ and $A_{1}=H_{1}$. Fix $n \geq 2$ and suppose that all the desired data has been chosen for all $k \leq n$ and all $(\mu, m) \in \widehat{A}_{k}$. We have to consider $(\lambda, l) \in \widehat{A}_{n+1}$ with $l>0$. As a model of the cell module $\Delta_{A_{n+1}}^{(\lambda, l)}$ we can take $\Delta_{A_{n-1}}^{(\lambda, l-1)} \otimes_{A_{n-1}} e_{n} A_{n+1}$, and for the generator of the cell module we can take $\delta_{A_{n+1}}^{(\lambda, l)}=\delta_{A_{n-1}}^{(\lambda, l-1)} \otimes_{A_{n-1}} e_{n}$.

Next we define $\alpha_{(\lambda, l)}$. According to [13, Sect. 4],

$$
\begin{aligned}
A_{n+1}^{\unrhd(\lambda, l)} & =A_{n+1} A_{n-1}^{\unrhd(\lambda, l-1)} e_{n} A_{n+1} \\
& \cong A_{n+1} e_{n} \otimes_{A_{n-1}} A_{n-1}^{\unrhd(\lambda, l-1)} \otimes_{A_{n-1}} e_{n} A_{n+1},
\end{aligned}
$$

as $A_{n+1}-A_{n+1}$ bimodules, with the isomorphism determined by $a_{1} x e_{n} a_{2} \mapsto a_{1} e_{n} \otimes x \otimes$ $e_{n} a_{2}$. Similarly

$$
\begin{aligned}
A_{n+1}^{\triangleright(\lambda, l)} & =A_{n+1} A_{n-1}^{\triangleright(\lambda, l-1)} e_{n} A_{n+1} \\
& \cong A_{n+1} e_{n} \otimes_{A_{n-1}} A_{n-1}^{\triangleright(\lambda, l-1)} \otimes_{A_{n-1}} e_{n} A_{n+1} .
\end{aligned}
$$

Moreover, we have an isomorphism

$$
\varphi: A_{n+1}^{\unrhd(\lambda, l)} / A_{n+1}^{\triangleright(\lambda, l)} \rightarrow A_{n+1} e_{n} \otimes_{A_{n-1}}\left(A_{n-1}^{\unrhd(\lambda, l-1)} / A_{n-1}^{\triangleright(\lambda, l-1)}\right) \otimes_{A_{n-1}} e_{n} A_{n+1},
$$

determined by

$$
\varphi\left(a_{1} x e_{n} a_{2}+A_{n+1}^{\triangleright(\lambda, l)}\right)=a_{1} e_{n} \otimes\left(x+A_{n-1}^{\triangleright(\lambda, l-1)}\right) \otimes e_{n} a_{2} .
$$

We identify $\left(e_{n} A_{n+1}\right)^{*}$ with $A_{n+1} e_{n}$ (as $A_{n+1}-A_{n-1}$ bimodules). Thus

$$
\left(\Delta_{A_{n+1}}^{(\lambda, l)}\right)^{*}=\left(\Delta_{A_{n-1}}^{(\lambda, l-1)} \otimes_{A_{n-1}} e_{n} A_{n+1}\right)^{*}=A_{n+1} e_{n} \otimes_{A_{n-1}}\left(\Delta_{A_{n-1}}^{(\lambda, l-1)}\right)^{*} .
$$

We define

$$
\alpha_{(\lambda, l)}=\left(\operatorname{id}_{A_{n+1} e_{n}} \otimes \alpha_{(\lambda, l-1)} \otimes \operatorname{id}_{e_{n} A_{n+1}}\right) \circ \varphi
$$

Thus

$$
\begin{aligned}
\alpha_{(\lambda, l)}: A_{n+1}^{\unrhd(\lambda, l)} / A_{n+1}^{\triangleright(\lambda, l)} & \rightarrow A_{n+1} e_{n} \otimes_{A_{n-1}}\left(\Delta_{A_{n-1}}^{(\lambda, l-1)}\right)^{*} \otimes_{R} \Delta_{A_{n-1}}^{(\lambda, l-1)} \otimes_{A_{n-1}} e_{n} A_{n+1} \\
& =\left(\Delta_{A_{n+1}}^{(\lambda, l)}\right)^{*} \otimes_{R} \Delta_{A_{n+1}}^{(\lambda, l)} .
\end{aligned}
$$

Now one can check that $* \circ \alpha_{(\lambda, l)}=\alpha_{(\lambda, l)} \circ *$.

Note that $c_{(\lambda, l-1)} e_{n} \in A_{n-1}^{\unrhd(\lambda, l-1)} e_{n} \subseteq A_{n+1}^{\unrhd(\lambda, l)}$ and

$$
\alpha_{(\lambda, l)}\left(c_{(\lambda, l-1)} e_{n}+A_{n+1}^{\triangleright(\lambda, l)}\right)=\left(e_{n} \otimes\left(\delta_{A_{n-1}}^{(\lambda, l-1)}\right)^{*}\right) \otimes\left(\delta_{A_{n-1}}^{(\lambda, l-1)} \otimes e_{n}\right)=\left(\delta_{A_{n+1}}^{(\lambda, l)}\right)^{*} \otimes \delta_{A_{n+1}}^{(\lambda, l)},
$$

so we can take $c_{(\lambda, l)}=c_{(\lambda, l-1)} e_{n}$. 
Let us restate this last observation, replacing $n+1$ by $n$. We have shown that if $(\lambda, l) \in \widehat{A}_{n}$ and $l>0$, then (we can take)

$$
c_{(\lambda, l)}=c_{(\lambda, l-1)} e_{n-1}
$$

By induction, we have

$$
c_{(\lambda, l)}=c_{(\lambda, 0)} e_{n-2 l+1} e_{n-2 l+3} \cdots e_{n-1} .
$$

Expressions of this form will appear again, so we establish the notation

$$
e_{n-1}^{(l)}= \begin{cases}1 & \text { if } l=0 \\ \underbrace{e_{n-2 l+1} e_{n-2 l+3} \cdots e_{n-1}}_{l \text { factors }} & \text { if } l=1, \ldots,\lfloor n / 2\rfloor, \text { and } \\ 0 & \text { if } l>\lfloor n / 2\rfloor .\end{cases}
$$

With this notation, we have

$$
c_{(\lambda, l)}=c_{(\lambda, 0)} e_{n-1}^{(l)} .
$$

\subsection{Branching Factors}

We continue to work with a pair of towers of algebras (5.1) satisfying the standing assumptions of Section 5.3.

We know already that both of the towers $\left(H_{n}\right)_{n \geq 0}$ and $\left(A_{n}\right)_{n \geq 0}$ are strongly coherent towers of cyclic cellular algebras, with $H_{n}^{F}$ and $A_{n}^{F}$ split semisimple for all $n$. Therefore, the analysis of Section 3.5, concerning branching factors and path bases, is applicable to both towers. We will show that the branching factors and path bases for the tower $\left(A_{n}\right)_{n \geq 0}$ can be computed by explicit formulas from those for the tower $\left(H_{n}\right)_{n \geq 0}$.

We suppose that we have chosen once and for all the following data for the tower $\left(H_{n}\right)_{n \geq 0}$, following observations (1) and (2), in Section 3.4:

(1) A generator $\delta_{H_{n}}^{\lambda}$ of the cell module $\Delta_{H_{n}}^{\lambda}$ for each $n$ and each $\lambda \in \widehat{H}_{n}$.

(2) Order preserving cell filtrations of $\operatorname{Res}_{H_{n}}^{H_{n+1}}\left(\Delta_{H_{n+1}}^{\mu}\right)$ and of $\operatorname{Ind}_{H_{n}}^{H_{n+1}}\left(\Delta_{H_{n}}^{\lambda}\right)$ for all $n$ and for all $\lambda \in \widehat{H}_{n}$ and $\mu \in \widehat{H}_{n+1}$,

$$
\{0\}=M_{0} \stackrel{\lambda^{(1)}}{\subseteq} M_{1} \stackrel{\lambda^{(2)}}{\subseteq} \cdots \stackrel{\lambda^{(r)}}{\subseteq} M_{r}=\operatorname{Res}_{H_{n}}^{H_{n+1}}\left(\Delta_{H_{n+1}}^{\mu}\right)
$$

and

$$
\{0\}=N_{0} \stackrel{\mu^{(1)}}{\subseteq} N_{1} \stackrel{\mu^{(2)}}{\subseteq} \cdots \stackrel{\mu^{(p)}}{\subseteq} N_{p}=\operatorname{Ind}_{H_{n}}^{H_{n+1}}\left(\Delta_{H_{n}}^{\lambda}\right) .
$$

(3) Branching factors $d_{\lambda \rightarrow \mu}^{(n+1)}$ and $u_{\lambda \rightarrow \mu}^{(n+1)}$ in $H_{n+1}$, for each $n$ and for each $\lambda \in \widehat{H}_{n}$ and $\mu \in \widehat{H}_{n+1}$ such that $\lambda \rightarrow \mu$ in $\widehat{H}$, with the property that

$$
\delta_{H_{n+1}}^{\mu} d_{\lambda^{(j)} \rightarrow \mu}^{(n+1)}+M_{j-1} \text { is a generator of } M_{j} / M_{j-1} \cong \Delta_{H_{n}}^{\lambda^{(j)}},
$$

and

$$
\delta_{H_{n}}^{\lambda} \otimes u_{\lambda \rightarrow \mu^{(i)}}^{(n+1)}+N_{i-1} \text { is a generator of } N_{i} / N_{i-1} \cong \Delta_{H_{n+1}}^{\mu^{(i)}} .
$$

(4) For each $n, \lambda$ and $\mu$, arbitrary liftings $\bar{u}_{\lambda \rightarrow \mu}^{(n+1)} \in \pi_{n+1}^{-1}\left(u_{\lambda \rightarrow \mu}^{(n+1)}\right)$ and $\bar{d}_{\lambda \rightarrow \mu}^{(n+1)} \in \pi_{n+1}^{-1}\left(d_{\lambda \rightarrow \mu}^{(n+1)}\right)$.

Since $\left(A_{n}\right)_{n \geq 0}$ is a strongly coherent tower of cyclic cellular algebras with each $A_{n}^{F}$ split semisimple, we know that there exist order preserving cell filtrations of $\operatorname{Res}_{A_{n}}^{A_{n+1}(\Delta)}$ for each cell module $\Delta$ of $A_{n+1}$ and of $\operatorname{Ind}_{A_{n}}^{A_{n+1}}(\Delta)$ for each cell module $\Delta$ of $A_{n}$, and 
there exist branching factors $d_{(\lambda, l) \rightarrow(\mu, m)}^{(n+1)}$ and $u_{(\lambda, l) \rightarrow(\mu, m)}^{(n+1)}$ in $A_{n+1}$ associated to each edge $(\lambda, l) \rightarrow(\mu, m)$ in the branching diagram $\widehat{A}$, with properties analogous to those listed above.

Neither the cell filtrations nor the branching factors are canonical. However, it was shown in [13] that cell filtrations of the induced and restricted modules for the tower $\left(A_{n}\right)$ can be obtained recursively, based on the cell filtrations of induced and restricted modules for the tower $\left(H_{n}\right)$. We will show here that the branching factors for the tower $\left(A_{n}\right)$ can also be chosen to satisfy recursive relations, so that they are determined completely by the liftings $\bar{u}_{\lambda \rightarrow \mu}^{(n+1)}$ and $\bar{d}_{\lambda \rightarrow \mu}^{(n+1)}$ of the branching factors for the tower $\left(H_{n}\right)$.

Each of the statements in the following theorem should be interpreted as applying whenever they make sense. For example, in statement (2), the branching factor $d_{(\lambda, l) \rightarrow(\mu, m+1)}^{(n+1)}$ makes sense when $n \geq 1,(\lambda, l) \in \widehat{A}_{n},(\mu, m+1) \in \widehat{A}_{n+1}$, and $(\lambda, l) \rightarrow(\mu, m+1)$ in the branching diagram $\widehat{A}$. This implies that $(\mu, m) \in \widehat{A}_{n-1}$ and $(\mu, m) \rightarrow(\lambda, l)$ in $\widehat{A}$, so that the branching factor $u_{(\mu, m) \rightarrow(\lambda, l)}^{(n)}$ also makes sense.

Theorem 5.6 The branching factors for the tower $\left(A_{n}\right)_{n \geq 0}$ can be chosen to satisfy:

(1) $d_{(\lambda, 0) \rightarrow(\mu, 0)}^{(n+1)}=\bar{d}_{\lambda \rightarrow \mu}^{(n+1)}$.

(2) $\quad d_{(\lambda, l) \rightarrow(\mu, m+1)}^{(n+1)}=u_{(\mu, m) \rightarrow(\lambda, l)}^{(n)}$.

(3) $u_{(\lambda, 0) \rightarrow(\mu, 0)}^{(n+1)}=\bar{u}_{\lambda \rightarrow \mu}^{(n+1)}$.

(4) $u_{(\lambda, l) \rightarrow(\mu, m+1)}^{(n+1)}=d_{(\mu, m) \rightarrow(\lambda, l)}^{(n)} e_{n}$.

Proof To prove this result, we have to look into, and add some detail to, the proof in $[13,14]$ that the tower $\left(A_{n}\right)$ is strongly coherent.

First we consider branching factors for reduced modules. The argument is an elaboration of the proof of [13, Proposition 4.10]. Let $n \geq 0$. Consider a cell module $\Delta_{A_{n+1}}^{(\mu, 0)}$ of $A_{n+1}$. We identify $\Delta_{A_{n+1}}^{(\mu, 0)}$ with the cell module $\Delta_{H_{n+1}}^{\mu}$ of $H_{n+1}$, and we identify the chosen generators of these modules, $\delta_{A_{n+1}}^{(\mu, 0)}$ with $\delta_{H_{n+1}}^{\mu}$. It follows from Axiom (6) that $J_{n} \subseteq J_{n+1}$ and hence

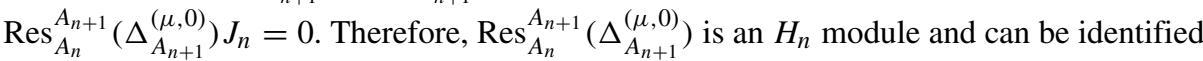
with $\operatorname{Res}_{H_{n}}^{H_{n+1}}\left(\Delta_{H_{n+1}}^{\mu}\right)$. Consider the chosen cell filtration of $\operatorname{Res}_{H_{n}}^{H_{n+1}}\left(\Delta_{H_{n+1}}^{\mu}\right)$

$$
\{0\} \subseteq M_{1} \subseteq M_{1} \subseteq \cdots \subseteq M_{r}=\operatorname{Res}_{H_{n}}^{H_{n+1}}\left(\Delta_{H_{n+1}}^{\mu}\right),
$$

with $M_{j} / M_{j-1} \cong \Delta_{H_{n}}^{\lambda^{(j)}}$ for each $j$. The isomorphism $M_{j} / M_{j-1} \rightarrow \Delta_{H_{n}}^{\lambda^{(j)}}$ maps $\delta_{H_{n+1}}^{\mu} d_{\lambda^{(j)} \rightarrow \mu}^{(n+1)}+M_{j-1}$ to $\delta_{H_{n}}^{\lambda^{(j)}}$. But we identify $\Delta_{H_{n}}^{\lambda^{(j)}}$ with $\Delta_{A_{n}}^{\left(\lambda^{(j)}, 0\right)}$ and $\delta_{H_{n}}^{\lambda^{(j)}}$ with $\delta_{A_{n}}^{\left(\lambda^{(j)}, 0\right)}$, so the isomorphism sends $\delta_{A_{n+1}}^{(\mu, 0)} \bar{d}_{\lambda^{(j)} \rightarrow \mu}^{(n+1)}+M_{j-1}=\delta_{H_{n+1}}^{\mu} d_{\lambda^{(j)} \rightarrow \mu}^{(n+1)}+M_{j-1}$ to $\delta_{A_{n}}^{\left(\lambda^{(j)}, 0\right)}$. Thus we can choose $d_{(\lambda(j), 0) \rightarrow(\mu, 0)}^{(n+1)}$ to be $\bar{d}_{\lambda^{(j)} \rightarrow \mu}^{(n+1)}$. This proves point (1).

Next, let $n \geq 1$ and consider a cell module

$$
\Delta=\Delta_{A_{n+1}}^{(\mu, m+1)}=\Delta_{A_{n-1}}^{(\mu, m)} \otimes_{A_{n-1}} e_{n} A_{n}
$$

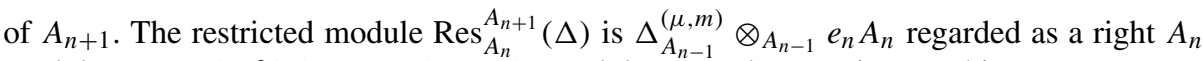
module. But $e_{n} A_{n} \cong A_{n}$ as an $A_{n-1}-A_{n}$ module, so we have an isomorphism

$$
\varphi: \Delta_{A_{n-1}}^{(\mu, m)} \otimes_{A_{n-1}} e_{n} A_{n} \rightarrow \Delta_{A_{n-1}}^{(\mu, m)} \otimes_{A_{n-1}} A_{n}=\operatorname{Ind}_{A_{n-1}}^{A_{n}}\left(\Delta_{A_{n-1}}^{(\mu, m)}\right),
$$


defined by $\varphi\left(x \otimes e_{n} a\right)=x \otimes a$. We suppose we already have a chosen cell filtration of $\operatorname{Ind}_{A_{n-1}}^{A_{n}}\left(\Delta_{A_{n-1}}^{(\mu, m)}\right)$,

$$
\{0\} \subseteq N_{1} \subseteq N_{2} \subseteq \cdots \subseteq N_{s}=\operatorname{Ind}_{A_{n-1}}^{A_{n}}\left(\Delta_{A_{n-1}}^{(\mu, m)}\right),
$$

with isomorphisms $N_{j} / N_{j-1} \rightarrow \Delta_{A_{n}}^{\left(\lambda^{(j)}, l_{j}\right)}$, as well as branching factors $u_{(\mu, m) \rightarrow\left(\lambda^{(j)}, l_{j}\right)}^{(n)}$ such that the isomorphism $N_{j} / N_{j-1} \rightarrow \Delta_{A_{n}}^{\left(\lambda^{(j)}, l_{j}\right)}$ takes $\delta_{A_{n-1}}^{(\mu, m)} \otimes_{A_{n-1}} u_{(\mu, m) \rightarrow\left(\lambda^{(j)}, l_{j}\right)}^{(n)}+N_{j-1}$ to $\delta_{A_{n}}^{\left(\lambda^{(j)}, l_{j}\right)}$. Pulling all this data back via $\varphi$, we have a cell filtration of $\operatorname{Res}_{A_{n}}^{A_{n+1}}(\Delta)$,

$$
\{0\} \subseteq N_{1}^{\prime} \subseteq N_{2}^{\prime} \subseteq \cdots \subseteq N_{s}^{\prime}=\operatorname{Res}(\Delta)
$$

with isomorphisms $\varphi_{j}: N_{j}^{\prime} / N_{j-1}^{\prime} \rightarrow \Delta_{A_{n}}^{\left(\lambda^{(j)}, l_{j}\right)}$ taking $\delta_{A_{n-1}^{(\mu, m)}}^{\left(\otimes_{A_{n-1}}\right.} e_{n} u_{(\mu, m) \rightarrow\left(\lambda^{(j)}, l_{j}\right)}^{(n)}+$ $N_{j-1}^{\prime}$ to $\delta_{A_{n}}^{\left(\lambda^{(j)}, l_{j}\right)}$. But $\delta_{A_{n-1}}^{(\mu, m)} \otimes_{A_{n-1}} e_{n}$ is the generator $\delta_{A_{n+1}}^{(\mu, m+1)}$ of $\Delta$. Thus

$$
\varphi_{j}: \delta_{A_{n+1}}^{(\mu, m+1)} u_{(\mu, m) \rightarrow\left(\lambda^{(j)}, l_{j}\right)}^{(n)}+N_{j-1}^{\prime} \mapsto \delta_{A_{n}}^{\left(\lambda^{(j)}, l_{j}\right)} .
$$

This means that we can choose $u_{(\mu, m) \rightarrow\left(\lambda^{(j)}, l_{j}\right)}^{(n)}$ for $d_{\left(\lambda^{(j)}, l_{j}\right) \rightarrow(\mu, m+1)}^{(n+1)}$. This proves point (2).

Next we turn to the branching factors for induced modules. Statement (3) is evident when $n=0$ since $A_{0}=H_{0}=R$ and $A_{1}=H_{1}$. Statement (4) only makes sense when $n \geq 1$, so it remains to verify both statements (3) and (4) for $n \geq 1$. The argument is an elaboration of the proof of Proposition 4.14 in [13].

Let $n \geq 1$ and let $\Delta$ be a cell module of $A_{n}$. According to [13, Proposition 4.14], $\Delta \otimes_{A_{n}}$ $J_{n+1}$ embeds in $\operatorname{Ind}_{A_{n}}^{A_{n+1}}(\Delta)$ and the quotient $\operatorname{Ind}_{A_{n}}^{A_{n+1}}(\Delta) /\left(\Delta \otimes_{A_{n}} J_{n+1}\right)$ is isomorphic to $\Delta \otimes_{A_{n}} H_{n+1}$. Moreover, both of the $A_{n+1}$-modules $\Delta \otimes_{A_{n}} J_{n+1}$ and $\Delta \otimes_{A_{n}} H_{n+1}$ have

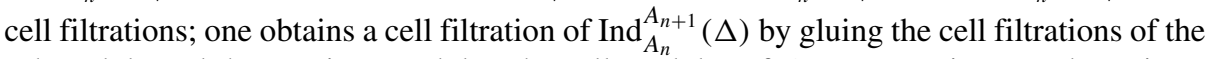
submodule and the quotient module. The cell modules of $A_{n+1}$ appearing as subquotients of the cell filtration of $\Delta \otimes_{A_{n}} J_{n+1}$ are of the form $\Delta_{A_{n+1}}^{(\mu, m)}$ with $m>0$; that is, they are cell modules of the cellular ideal $J_{n+1}$. The cell modules appearing as subquotients of the cell filtration of $\Delta \otimes_{A_{n}} H_{n+1}$ are of the form $\Delta_{A_{n+1}}^{(\mu, 0)}$; that is, they are cell modules of the quotient algebra $H_{n+1}$.

Now consider in particular a cell module $\Delta_{A_{n}}^{(\lambda, 0)}$ of $A_{n}$ for $n \geq 1$. According to the previous paragraph, to find the branching factors $u_{(\lambda, 0) \rightarrow(\mu, 0)}^{(n+1)}$ with $\mu \in \widehat{H}_{n+1}$, we have only to construct a particular cell filtration of $\Delta_{A_{n}}^{(\lambda, 0)} \otimes_{A_{n}} H_{n+1}$. We identify $\Delta_{A_{n}}^{(\lambda, 0)}$ with the cell module $\Delta_{H_{n}}^{\lambda}$ of $H_{n}$, and $\Delta_{A_{n}}^{(\lambda, 0)} \otimes_{A_{n}} H_{n+1}$ with $\Delta_{H_{n}}^{\lambda} \otimes_{H_{n}} H_{n+1}=\operatorname{Ind}_{H_{n}}^{H_{n+1}}\left(\Delta_{H_{n}}^{\lambda}\right)$. The remainder of the proof of statement (3) proceeds by considering the chosen cell filtration of $\operatorname{Ind}_{H_{n}}^{H_{n+1}}\left(\Delta_{H_{n}}^{\lambda}\right)$ and the associated branching factors $u_{\lambda \rightarrow \mu}^{(n+1)}$; the proof is similar to the proof of statement (1).

Finally, let $n \geq 1$ and consider a cell module $\Delta=\Delta_{A_{n}}^{(\lambda, l)}$ of $A_{n}$. Write $\operatorname{Res}(\Delta)$ for $\operatorname{Res}_{A_{n-1}}^{A_{n}}(\Delta)$. To find the branching factors $u_{(\lambda, l) \rightarrow(\mu, m+1)}^{(n+1)}$, we have to construct a particular cell filtration of $\Delta \otimes_{A_{n}} J_{n+1}$. By axiom (7) and [13, Corollary 4.6], we have

$$
J_{n+1}=A_{n} e_{n} A_{n} \cong A_{n} e_{n} \otimes_{A_{n-1}} e_{n} A_{n},
$$


as $A_{n}-A_{n+1}$ bimodules, the isomorphism being given by $a_{1} e_{n} a_{2} \mapsto a_{1} e_{n} \otimes_{A_{n-1}} e_{n} a_{2}$. We have $A_{n} e_{n} \cong A_{n}$ as an $A_{n}-A_{n-1}$ bimodule, so

$$
\begin{aligned}
\Delta \otimes_{A_{n}} J_{n+1} & \cong \Delta \otimes_{A_{n}} A_{n} e_{n} \otimes_{A_{n-1}} e_{n} A_{n} \\
& \cong \Delta \otimes_{A_{n}} A_{n} \otimes_{A_{n-1}} e_{n} A_{n} \\
& \cong \operatorname{Res}(\Delta) \otimes_{A_{n-1}} e_{n} A_{n} .
\end{aligned}
$$

The composite isomorphism $\varphi: \Delta \otimes_{A_{n}} J_{n+1} \rightarrow \operatorname{Res}(\Delta) \otimes_{A_{n-1}} e_{n} A_{n}$ is given by $\varphi\left(x \otimes_{A_{n}} a_{1} e_{n} a_{2}\right)=x a_{1} \otimes_{A_{n-1}} e_{n} a_{2}$. In particular, $\varphi\left(x \otimes_{A_{n}} e_{n}\right)=x \otimes_{A_{n-1}} e_{n}$. We assume that we have a chosen cell filtration of $\operatorname{Res}(\Delta)$,

$$
\{0\} \subseteq M_{1} \subseteq M_{2} \subseteq \cdots \subseteq M_{t}=\operatorname{Res}(\Delta),
$$

with isomorphisms $M_{j} / M_{j-1} \rightarrow \Delta_{A_{n-1}}^{\left(\mu^{(j)}, m_{j}\right)}$ and we have chosen branching factors $d_{\left(\mu^{(j)}, m_{j}\right) \rightarrow(\lambda, l)}^{(n)}$ such that the isomorphism $M_{j} / M_{j-1} \rightarrow \Delta_{A_{n-1}}^{\left(\mu^{(j)}, m_{j}\right)}$ takes $\delta_{A_{n}}^{(\lambda, l)} d_{\left(\mu^{(j)}, m_{j}\right) \rightarrow(\lambda, l)}^{(n)}+M_{j-1}$ to $\delta_{A_{n-1}}^{\left(\mu^{(j)}, m_{j}\right)}$. By [13, Lemma 4.12], $M_{j-1} \otimes_{A_{n-1}} e_{n} A_{n}$ embeds in $M_{j} \otimes_{A_{n-1}} e_{n} A_{n}$ for each $j$, and the quotient is isomorphic to

$$
M_{j} / M_{j-1} \otimes_{A_{n-1}} e_{n} A_{n} \cong \Delta_{A_{n-1}}^{\left(\mu^{(j)}, m_{j}\right)} \otimes_{A_{n-1}} e_{n} A_{n}=\Delta_{A_{n+1}}^{\left(\mu^{(j)}, m_{j}+1\right)} .
$$

Writing $M_{j}^{\prime}=M_{j} \otimes_{A_{n-1}} e_{n} A_{n}$, we obtain a cell filtration of $\operatorname{Res}(\Delta) \otimes_{A_{n-1}} e_{n} A_{n}$,

$$
\{0\} \subseteq M_{1}^{\prime} \subseteq M_{2}^{\prime} \subseteq \cdots \subseteq M_{t}^{\prime}=\operatorname{Res}(\Delta) \otimes_{A_{n-1}} e_{n} A_{n},
$$

with isomorphisms $M_{j}^{\prime} / M_{j-1}^{\prime} \rightarrow \Delta_{A_{n+1}}^{\left(\mu^{(j)}, m_{j}+1\right)}$ taking $\delta_{A_{n}}^{(\lambda, l)} d_{\left(\mu^{(j)}, m_{j}\right) \rightarrow(\lambda, l)}^{(n)} \otimes_{A_{n-1}} e_{n}+M_{j-1}^{\prime}$ to $\delta_{A_{n-1}}^{\left(\mu^{(j)}, m_{j}\right)} \otimes_{A_{n-1}} e_{n}=\delta_{A_{n+1}}^{\left(\mu^{(j)}, m_{j}+1\right)}$. Pulling back this data via the isomorphism $\varphi: \Delta \otimes_{A_{n}}$

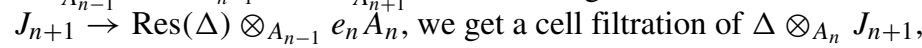

$$
\{0\} \subseteq M_{1}^{\prime \prime} \subseteq M_{2}^{\prime \prime} \subseteq \cdots \subseteq M_{t}^{\prime \prime}=\Delta \otimes_{A_{n}} J_{n+1},
$$

with isomorphisms $M_{j}^{\prime \prime} / M_{j-1}^{\prime \prime} \rightarrow \Delta_{A_{n+1}}^{\left(\mu^{(j)}, m_{j}+1\right)}$ taking

$$
\delta_{A_{n}}^{(\lambda, l)} d_{\left(\mu^{(j)}, m_{j}\right) \rightarrow(\lambda, l)}^{(n)} \otimes_{A_{n}} e_{n}+M_{j-1}^{\prime \prime}=\delta_{A_{n}}^{(\lambda, l)} \otimes_{A_{n}} d_{\left(\mu^{(j)}, m_{j}\right) \rightarrow(\lambda, l)}^{(n)} e_{n}+M_{j-1}^{\prime \prime}
$$

to $\delta_{A_{n+1}}^{\left(\mu^{(j)}, m_{j}+1\right)}$. We conclude that we can take

$$
u_{(\lambda, l) \rightarrow\left(\mu^{(j)}, m_{j}+1\right)}^{(n+1)}=d_{\left(\mu^{(j)}, m_{j}\right) \rightarrow(\lambda, l)}^{(n)} e_{n},
$$

which proves point (4), and completes the proof of the theorem.

Next we apply the recursion of Theorem 5.6 to obtained closed formulas for the branching factors for the tower $\left(A_{n}\right)_{n \geq 0}$. Since the branching diagram $\widehat{A}$ is obtained by reflections from the branching diagram $\widehat{H}$, it follows that $(\lambda, l) \rightarrow(\mu, m)$ only if $m \in\{l, l+1\}$; in particular, $(\lambda, l) \rightarrow(\mu, 0)$ only if $l=0$. Moreover, $(\lambda, l) \rightarrow(\mu, l)$ in $\widehat{A}$ if and only if $\lambda \rightarrow \mu$ in $\widehat{H}$, and $(\lambda, l) \rightarrow(\mu, l+1)$ in $\widehat{A}$ if and only if $\mu \rightarrow l$ in $\widehat{H}$.

Theorem 5.7 The branching factors for the tower $\left(A_{n}\right)_{n \geq 0}$ can be chosen to satisfy:

(1) $d_{(\lambda, l) \rightarrow(\mu, l)}^{(n+1)}=\bar{d}_{\lambda \rightarrow \mu}^{(n+1-2 l)} e_{n-1}^{(l)}$.

(2) $u_{(\lambda, l) \rightarrow(\mu, l)}^{(n+1)}=\bar{u}_{\lambda \rightarrow \mu}^{(n+1-2 l)} e_{n}^{(l)}$. 
(3) $d_{(\lambda, l) \rightarrow(\mu, l+1)}^{(n+1)}=\bar{u}_{\mu \rightarrow \lambda}^{(n-2 l)} e_{n-1}^{(l)}$.

(4) $u_{(\lambda, l) \rightarrow(\mu, l+1)}^{(n+1)}=\bar{d}_{\mu \rightarrow \lambda}^{(n-2 l)} e_{n}^{(l+1)}$.

Proof We suppose that the branching factors are determined by the recursive formulas of Theorem 5.6.

For part (1), the formula is given by Theorem 5.6, part (1) if $l=0$. Assume $l>0$ and observe

$$
d_{(\lambda, l) \rightarrow(\mu, l)}^{(n+1)}=u_{(\mu, l-1) \rightarrow(\lambda, l)}^{(n)}=d_{(\lambda, l-1) \rightarrow(\mu, l-1)}^{(n-1)} e_{n-1} ;
$$

Repeating this a total of $l$ times, we get

$$
d_{(\lambda, l) \rightarrow(\mu, l)}^{(n+1)}=d_{(\lambda, 0) \rightarrow(\mu, 0)}^{(n+1-2 l)} e_{n+1-2 l} \cdots e_{n-3} e_{n-1}=\bar{d}_{\lambda \rightarrow \mu}^{(n+1-2 l)} e_{n-1}^{(l)} .
$$

The proof of part (2) is similar. For part (3), we have

$$
d_{(\lambda, l) \rightarrow(\mu, l+1)}^{(n+1)}=u_{(\mu, l) \rightarrow(\lambda, l)}^{(n)},
$$

and we apply part (2) to get the desired formula. For part (4),

$$
u_{(\lambda, l) \rightarrow(\mu, l+1)}^{(n+1)}=d_{(\mu, l) \rightarrow(\lambda, l)}^{(n)} e_{n} .
$$

Apply part (1) to get

$$
u_{(\lambda, l) \rightarrow(\mu, l+1)}^{(n+1)}=d_{(\mu, l) \rightarrow(\lambda, l)}^{(n)} e_{n}=\bar{d}_{\mu \rightarrow \lambda}^{(n-2 l)} e_{n-2}^{(l)} e_{n}=\bar{d}_{\mu \rightarrow \lambda}^{(n-2 l)} e_{n}^{(l+1)} .
$$

\section{Applications}

We will apply our results to the following examples: the BMW algebras, the Brauer algebras, the partition algebras, and the Jones-Temperley-Lieb algebras. For each example, let $R_{0}$ denote the generic ground ring and let $R=R_{0}\left[\delta^{-1}\right]$, where $e_{1}^{2}=\delta e_{1}$. We show that our results apply to the algebras defined over $R$, and we give explicit Murphy bases for the algebras.

We are then able to check, by a computation specific to each algebra, that the Murphy bases are, in fact, bases for the algebras defined over the generic ground ring $R_{0}$.

\subsection{Preliminaries on Tangle Diagrams}

Several of our examples involve tangle diagrams in the rectangle $\mathcal{R}=[0,1] \times[0,1]$. Fix points $a_{i} \in[0,1], i \geq 1$, with $0<a_{1}<a_{2}<\cdots$. Write $\boldsymbol{i}=\left(a_{i}, 1\right)$ and $\overline{\boldsymbol{i}}=\left(a_{i}, 0\right)$.

Recall that a knot diagram means a collection of piecewise smooth closed curves in the plane which may have intersections and self-intersections, but only simple transverse intersections. At each intersection or crossing, one of the two strands (curves) which intersect is indicated as crossing over the other.

An $(n, n)$-tangle diagram is a piece of a knot diagram in $\mathcal{R}$ consisting of exactly $n$ topological intervals and possibly some number of closed curves, such that: (1) the endpoints of the intervals are the points $\mathbf{1}, \ldots, \boldsymbol{n}, \overline{\mathbf{1}}, \ldots, \overline{\boldsymbol{n}}$, and these are the only points of intersection of the family of curves with the boundary of the rectangle, and (2) each interval intersects the boundary of the rectangle transversally.

An $(n, n)$-Brauer diagram is a "tangle" diagram containing no closed curves, in which information about over and under crossings is ignored. Two Brauer diagrams are identified 
if the pairs of boundary points joined by curves is the same in the two diagrams. By convention, there is a unique $(0,0)-$ Brauer diagram, the empty diagram with no curves. For $n \geq 1$, the number of $(n, n)$-Brauer diagrams is $(2 n-1) ! !=(2 n-1)(2 n-3) \cdots(3)(1)$.

For any of these types of diagrams, we call $P=\{\mathbf{1}, \ldots, \boldsymbol{n}, \overline{\mathbf{1}}, \ldots, \overline{\boldsymbol{n}}\}$ the set of vertices of the diagram, $P^{+}=\{\mathbf{1}, \ldots, \boldsymbol{n}\}$ the set of top vertices, and $P^{-}=\{\overline{\mathbf{1}}, \ldots, \overline{\boldsymbol{n}}\}$ the set of bottom vertices. A curve or strand in the diagram is called a vertical or through strand if it connects a top vertex and a bottom vertex, and a horizontal strand if it connects two top vertices or two bottom vertices.

\subsection{Birman-Murakami-Wenzl Algebras}

The Birman-Murakami-Wenzl (BMW) algebras were introduced by Birman and Wenzl [1] and independently by Murakami [32] . The version of the presentation given here follows [31]. Cellularity of the BMW algebras was established in [8, 9, 43].

Definition 6.1 Let $S$ be an integral domain with invertible elements $z$ and $q$ and an element $\delta$ satisfying $z^{-1}-z=\left(q^{-1}-q\right)(\delta-1)$. The Birman-Murakami-Wenzl algebra $W_{n}=$ $W_{n}(S ; z, q, \delta)$ is the unital $S$-algebra with generators $g_{i}^{ \pm 1}$ and $e_{i}(1 \leq i \leq n-1)$ and relations:

(1) (Inverses) $g_{i} g_{i}^{-1}=g_{i}^{-1} g_{i}=1$.

(3) (Essential idempotent relation) $e_{i}^{2}=\delta e_{i}$.

(4) (Braid relations) $g_{i} g_{i+1} g_{i}=g_{i+1} g_{i} g_{i+1}$ and $g_{i} g_{j}=g_{j} g_{i}$ if $|i-j| \geq 2$.

(5) (Commutation relations) $g_{i} e_{j}=e_{j} g_{i}$ and $e_{i} e_{j}=e_{j} e_{i}$ if $|i-j| \geq 2$.

(6) (Tangle relations) $e_{i} e_{i \pm 1} e_{i}=e_{i}, g_{i} g_{i \pm 1} e_{i}=e_{i \pm 1} e_{i}$, and $e_{i} g_{i \pm 1} g_{i}=e_{i} e_{i \pm 1}$.

(7) (Kauffman skein relation) $g_{i}-g_{i}^{-1}=\left(q-q^{-1}\right)\left(1-e_{i}\right)$.

(8) (Untwisting relations) $g_{i} e_{i}=e_{i} g_{i}=z^{-1} e_{i}$, and $e_{i} g_{i \pm 1} e_{i}=z e_{i}$.

Morton and Wassermann [31] give a realization of the BMW algebra as an algebra of $(n, n)$-tangle diagrams modulo regular isotopy and the following Kauffman skein relations:

(1) Crossing relation:

$$
\searrow-\rangle^{\prime}=\left(q-q^{-1}\right)()(-\nearrow)
$$

(2) Untwisting relation:

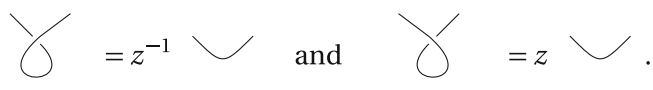

(3) Free loop relation: $T \cup \bigcirc=\delta T$, where $T \cup \bigcirc$ means the union of a tangle diagram $T$ and a closed loop having no crossings with $T$.

In the tangle picture, the generators $g_{i}$ and $e_{i}$ are represented by the diagrams

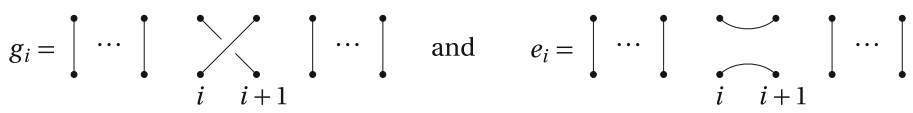

There is evidently a unital algebra homomorphism from $W_{n}$ to $W_{n+1}$ taking generators to generators; from the tangle realization, one can see that this homomorphism is injective, so 
$W_{n}$ is a subalgebra of $W_{n+1}$. The symmetry of the defining relations for $W_{n}$ ensures that the assignments

$$
g_{i}^{*}=g_{i}, \quad e_{i}^{*}=e_{i} .
$$

determine an involutory algebra anti-automorphism of $W_{n}$. In the tangle picture, the involution $*$ acts on tangles by flipping them over a horizontal line.

If $v \in \mathfrak{S}_{n}$ and $v=s_{i_{1}} s_{i_{2}} \cdots s_{i_{j}}$ is a reduced expression then the element $g_{v}=$ $g_{i_{1}} g_{i_{2}} \cdots g_{i_{j}}$ depends only on $v$. For $i, j=1,2, \ldots$, let

$$
g_{i, j}=\left\{\begin{array}{l}
g_{i} g_{i+1} \cdots g_{j-1}, \text { if } j \geq i, \\
g_{i-1} g_{i-2} \cdots g_{j}, \text { if } i>j .
\end{array}\right.
$$

Let $J_{n}$ denote the ideal $W_{n} e_{n-1} W_{n}$; in the tangle picture, this is the ideal spanned by tangle diagrams with at least one horizontal strand. The map $W_{n} / J_{n} \rightarrow \mathcal{H}_{n}=\mathcal{H}_{n}\left(S, q^{2}\right)$ determined by $g_{v}+J_{n} \mapsto T_{v}$, for $v \in \mathfrak{S}_{n}$, is an algebra isomorphism.

\subsubsection{The Murphy Basis}

The generic ground ring for the BMW algebras is

$$
R_{0}=\mathbb{Z}\left[z^{ \pm 1}, \boldsymbol{q}^{ \pm 1}, \boldsymbol{\delta}\right] /\left\langle z^{-1}-z=\left(\boldsymbol{q}^{-1}-\boldsymbol{q}\right)(\boldsymbol{\delta}-1)\right\rangle,
$$

where $z, \boldsymbol{q}$, and $\delta$ are indeterminants over $\mathbb{Z} . R_{0}$ is an integral domain whose field of fractions is $F \cong \mathbb{Q}(z, \boldsymbol{q})$, with

$$
\delta=\frac{z-z^{-1}}{\boldsymbol{q}-\boldsymbol{q}^{-1}}+1=\frac{(z+\boldsymbol{q})(\boldsymbol{q} z-1)}{\boldsymbol{z}\left(\boldsymbol{q}^{2}-1\right)} .
$$

Let $R=R_{0}\left[\boldsymbol{\delta}^{-1}\right]$, and write $W_{n}(R)$ for $W_{n}(R ; z, \boldsymbol{q}, \boldsymbol{\delta})$ and $\mathcal{H}_{n}(R)$ for $\mathcal{H}_{n}\left(R ; \boldsymbol{q}^{2}\right)$. It is observed in [13], Section 5.4, that the pair of towers $\left(W_{n}(R)\right)_{n \geq 0}$ and $\left(\mathcal{H}_{n}(R)\right)_{n \geq 0}$ satisfy the framework axioms (1)-(7) of Section 5.1. Axiom (8) holds by Corollary 4.10. Axiom (9) hold for $W_{n}(R)$, by the remarks at the end of Section 5.1. Finally, Axiom (10) holds by Corollary 4.3. Therefore, by Theorem 5.5, the tower of algebras $\left(W_{n}(R)\right)_{n \geq 0}$ is a strongly coherent tower of cyclic cellular algebras.

By the discussion in Section 5.4, the partially ordered set $\widehat{W}_{n}$ in the cell datum for $W_{n}(R)$ can be realized as

$$
\widehat{W}_{n}=\left\{(\lambda, l) \mid 0 \leq l \leq\lfloor n / 2\rfloor \text { and } \lambda \in \widehat{\mathcal{H}}_{n-2 l}\right\}
$$

with $(\lambda, l) \unrhd(\mu, m)$ if $l>m$ or if $l=m$ and $\lambda \triangleright \mu$ in $\widehat{\mathcal{H}}_{n-2 l}$. The branching diagram $\widehat{W}$ of the tower $\left(W_{n}\right)_{n \geq 0}$ is that obtained by reflections from $\widehat{\mathcal{H}}$ (= Young's lattice). Thus, the branching relation is $(\lambda, l) \rightarrow(\mu, m)$ only if $m \in\{l, l+1\} ;(\lambda, l) \rightarrow(\mu, l)$ if and only if $\lambda \rightarrow \mu$ in Young's lattice, and $(\lambda, l) \rightarrow(\mu, l+1)$ if and only if $\mu \rightarrow \lambda$ in Young's lattice.

For each $n \geq 0$ and for each $\mu \in \widehat{\mathcal{H}}_{n}$, define

$$
c_{(\mu, 0)}=\sum_{v \in \mathfrak{S}_{\mu}} q^{l(v)} g_{v}
$$

thus $c_{(\mu, 0)}$ is a preimage in $W_{n}$ of $m_{\mu} \in \mathcal{H}_{n}$ (defined in Eq. (4.2)). For $n \geq 2$ and $(\mu, m) \in$ $\widehat{W}_{n}$, let

$$
c_{(\mu, m)}=c_{(\mu, 0)} e_{n-1}^{(m)},
$$

where $e_{n-1}^{(m)}$ is defined in Eq. (5.4). 
Let $i \geq 1$ and $\lambda \in \widehat{\mathcal{H}}_{i-1}$ and $\mu \in \widehat{\mathcal{H}}_{i}$, with $\lambda \rightarrow \mu$ in $\widehat{\mathcal{H}}$. If $\mu=\lambda \cup\left\{\left(r, \mu_{r}\right)\right\}$, let $a=\sum_{j=1}^{r} \mu_{j}$, and define

$$
\bar{u}_{\lambda \rightarrow \mu}^{(i)}=g_{i, a} \sum_{k=0}^{\lambda_{r}} q^{k} g_{a, a-k} \quad \text { and } \quad \bar{d}_{\lambda \rightarrow \mu}^{(i)}=g_{a, i} .
$$

These are liftings in $W_{i}$ of the branching factors in the Hecke algebra $\mathcal{H}_{i}$, as determined in Corollary 4.5 and Corollary 4.19.

For $(\lambda, l) \in \widehat{W}_{i}$ and $(\mu, m) \in \widehat{W}_{i+1}$ with $(\lambda, l) \rightarrow(\mu, m)$, determine the branching factors $d_{(\lambda, l) \rightarrow(\mu, m)}^{(i+1)}$ according to the formulas of Theorem 5.7; for a path $\mathfrak{t} \in \widehat{W}_{n}^{(\lambda, l)}$, define $d_{\mathfrak{t}}$ to be the ordered product of these branching factors along the path $\mathfrak{t}$, as in Eq. (3.4). From Corollary 3.11 we obtain:

Proposition 6.2 Let $R_{0}$ denote the generic ground ring for the BMW algebras and let $R=$ $R_{0}\left[\boldsymbol{\delta}^{-1}\right]$. Let $W_{n}(R)=W_{n}(R ; \boldsymbol{z}, \boldsymbol{q}, \boldsymbol{\delta})$ denote the BMW algebra over $R$. For $n \geq 0$, the set

$$
\mathscr{W}_{n}=\left\{d_{\mathfrak{s}}^{*} c_{(\lambda, l)} d_{\mathfrak{t}} \mid \mathfrak{s}, \mathfrak{t} \in \widehat{W}_{n}^{(\lambda, l)},(\lambda, l) \in \widehat{W}_{n}\right\}
$$

is an $R$-basis for $W_{n}(R)$, and $\left(W_{n}(R), *, \widehat{W}_{n}, \unrhd, \mathscr{W}_{n}\right)$ is a cell datum for $W_{n}(R)$.

In the remainder of this section, we will show that the Murphy bases $\mathscr{W}_{n}$ are bases of the BMW algebras defined over the generic ground ring $R_{0}$. First note that the elements $d_{\mathfrak{s}}^{*} c_{(\lambda, l)} d_{\mathfrak{t}}$ are actually defined over $R_{0}$ and are linearly independent. The issue is to show that $\mathscr{W}_{n}$ spans the BMW algebra over $R_{0}$. To do this, we examine the transition matrix between a Morton-Wassermann basis of the BMW algebra and $\mathscr{W}_{n}$.

\subsubsection{Morton-Wassermann Tangle Bases}

We begin by describing the Morton-Wassermann tangle bases of the BMW algebras. We identify the BMW algebras with their tangle realizations, following [31].

To each $(n, n)$-tangle diagram $T$, associate a Brauer diagram conn $(T)$ by deleting the closed strands in $T$ and forgetting information about over and under crossings. Thus $\operatorname{conn}(T)$ has a strand connecting two vertices if and only if $T$ has a strand connecting the same two vertices.

Order the vertices of a tangle or Brauer diagram by $\mathbf{1}<\mathbf{2} \cdots<\boldsymbol{n}<\overline{\boldsymbol{n}}<\cdots<\overline{\mathbf{1}}$, that is, in clockwise order around the boundary of $\mathcal{R}$. The length $\ell(D)$ of a Brauer diagram $D$ is the minimal number of crossings of strands in a physical drawing of the diagram, that is, the number of 4-tuples of vertices $(a, b, c, d)$ such that $a<b<c<d$ and $(a, c)$ and $(b, d)$ are strands of $D$.

Definition 6.3 Say that an $(n, n)$-tangle diagram $T$ is layered with respect to some total ordering $\left(t_{1}, t_{2}, \ldots, t_{k}\right)$ of its strands, if (1) whenever $i<j$, every crossing of $t_{i}$ with $t_{j}$ is an over crossing, and (2) each individual strand of $T$ is unknotted, i.e. ambient isotopic to a strand with no self-crossings. Say that $T$ is layered if it is layered with respect to some total ordering of its strands. Say that a layered tangle diagram is simple if it has no closed strands and no strand has self-crossings.

Note that any simple layered tangle diagram $T$ is ambient isotopic to a simple layered tangle diagram in which any two distinct strands have at most one crossing; the number of crossings in such a representative of $T$ is the length of conn $(T)$. 
Theorem 6.4 ([31], Theorems 2.10 and 4.2) For each $(n, n)$-Brauer diagram D, chose a simple layered $(n, n)$-tangle diagram $T$ with $\operatorname{conn}(T)=D$. Then the resulting collection of tangle diagrams is a basis of the BMW algebra $W_{n}\left(R_{0}\right)$.

\section{Call any such basis a Morton-Wassermann tangle basis.}

Lemma 6.5 Let $T$ and $S$ be simple layered $(n, n)$-tangle diagrams with the same underlying Brauer diagram, $\operatorname{conn}(T)=\operatorname{conn}(S)=D$. Then $T-S$ is in the $\mathbb{Z}\left[\boldsymbol{q}-\boldsymbol{q}^{-1}\right]$-span of simple layered tangle diagrams with fewer than $\ell(D)$ crossings.

Proof Assume without loss of generality that the number of crossings of $T$ and of $S$ is the length of $D$. Suppose that $S$ is layered with respect to an ordering $\left(t_{1}, t_{2}, \ldots, t_{n}\right)$ of its strands and $T$ is layered with respect to an ordering $\left(t_{\pi(1)}, t_{\pi(2)}, \ldots, t_{\pi(n)}\right)$ for some permutation $\pi$ of $\{1,2, \ldots, n\}$. For brevity, say that $T$ is layered with respect to $\pi$. The permutation $\pi$ may not be unique, so assume that $\pi$ has been chosen with minimal length for the given tangle diagram $T$.

If $\pi$ is the identity permutation, then $T$ and $S$ are ambient isotopic, so represent the same element of $W_{n}$. Assume that $\pi$ is not the identity and assume inductively that the assertion holds when $T$ is replaced by a simple layered tangle diagram $T^{\prime}$ with $\operatorname{conn}\left(T^{\prime}\right)=D$, whenever $T^{\prime}$ is layered with respect to a permutation $\pi^{\prime}$ with $\ell\left(\pi^{\prime}\right)<\ell(\pi)$.

Since $\pi$ is not the identity permutation, there exists $i$ such that $\pi(i)>\pi(i+1)$. If the strands $t_{\pi(i)}$ and $t_{\pi(i+1)}$ do not cross, then $T$ is also layered with respect to the shorter permutation $\pi^{\prime}=(i, i+1) \circ \pi$, contradicting the choice of $\pi$ as having minimal length. Therefore $t_{\pi(i)}$ and $t_{\pi(i+1)}$ have a (unique) crossing, with $t_{\pi(i)}$ crossing over $t_{\pi(i+1)}$. Because $T$ is layered with respect to $\pi$ there is no third strand $t=t_{\pi(k)}$ such that $t_{\pi(i)}$ has an over crossing with $t$ and $t$ has an over crossing with $t_{\pi(i+1)}$. Let $U$ be the tangle diagram obtained by changing the crossing of $t_{\pi(i)}$ and $t_{\pi(i+1)}$, and let $T_{0}$ and $T_{\infty}$ be the two tangle diagrams obtained by smoothing this crossing. It follows that all three of these tangle diagrams are simple and layered, $T_{0}$ and $T_{\infty}$ have fewer than $\ell(D)$ crossings, and by the Kauffman skein relation,

$$
T=U+\left(\boldsymbol{q}-\boldsymbol{q}^{-1}\right)\left(T_{0}-T_{\infty}\right) .
$$

Since $U$ is layered with respect to $\pi^{\prime}=(i, i+1) \circ \pi$, with $\ell\left(\pi^{\prime}\right)=\ell(\pi)-1$, the conclusion follows from the induction hypothesis.

Proposition 6.6 Let $\mathcal{B}$ be a Morton-Wassermann tangle basis of $W_{n}\left(R_{0}\right)$ and let $T$ be a simple layered $(n, n)$-tangle diagram. The the coefficients of $T$ with respect to the basis $\mathcal{B}$ are in $\mathbb{Z}\left[\boldsymbol{q}-\boldsymbol{q}^{-1}\right]$. In fact, $T$ is in the $\mathbb{Z}\left[\boldsymbol{q}-\boldsymbol{q}^{-1}\right]$-span of basis elements with no more than $\ell(D)$ crossings, where $D=\operatorname{conn}(T)$.

Proof We can assume that the number of crossings of $T$ is $\ell(D)$, where $D=\operatorname{conn}(T)$. We proceed by induction on the number of crossings. If $T$ has no crossings, then $T$ is an element of $\mathcal{B}$, because up to ambient isotopy, there is a unique simple layered tangle diagram with underlying Brauer diagram $D$. Assume that $\ell(D)$ is positive and that the statement holds for all simple layered tangle diagrams with fewer than $\ell(D)$ crossings. There is a simple layered tangle diagram $S$ in $\mathcal{B}$ with $\operatorname{conn}(S)=D$. By the previous lemma, $T-S$ is a $\mathbb{Z}\left[\boldsymbol{q}-\boldsymbol{q}^{-1}\right]-$ linear combination of simple layered tangle diagrams with fewer than $\ell(D)$ crossings, and thus the result follows from the induction hypothesis. 
Corollary 6.7 The transition matrix between any two Morton-Wassermann tangle bases of $W_{n}$ has entries in $\mathbb{Z}\left[\boldsymbol{q}-\boldsymbol{q}^{-1}\right]$.

Lemma 6.8 Let $\mathcal{B}$ be a Morton-Wassermann tangle basis of $W_{n}\left(R_{0}\right)$. The matrix with respect to $\mathcal{B}$ of left or right multiplication by $g_{i}$ or $g_{i}^{-1}$ has entries in $\mathbb{Z}\left[z^{ \pm 1},\left(\boldsymbol{q}-\boldsymbol{q}^{-1}\right)\right]$.

Proof Let $T$ be an element of $\mathcal{B}$; assume without loss of generality that the number of crossings of $T$ is $\ell(D)$ where $D$ denotes conn $(T)$. We have to show that $T g_{i}$ is in the $\mathbb{Z}\left[z^{ \pm 1},\left(\boldsymbol{q}-\boldsymbol{q}^{-1}\right)\right]$-span of $\mathcal{B}$. We proceed by induction on the number of crossings of $T$. If $T$ has no crossings, then $T g_{i}$ is simple and layered, so the assertion follows from Proposition 6.6 .

Assume that $\ell(D)>0$ and that the assertion holds when $T$ is replaced by an element of $\mathcal{B}$ with fewer crossings. If the vertices $\overline{\boldsymbol{i}}$ and $\overline{\boldsymbol{i}+\mathbf{1}}$ of $T$ are connected by a strand, then $T g_{i}=z^{-1} T$, so we are done. Otherwise, let $s$ and $t$ denote the distinct strands of $T$ incident on the vertices $\overline{\boldsymbol{i}}$ and $\overline{\boldsymbol{i}+\mathbf{1}}$. Let $S$ be a simple layered tangle diagram such that $\operatorname{conn}(S)=D, S$ has $\ell(D)$ crossings, and $S$ is layered with respect to an ordering $(t, s, \ldots)$ of the strands. Then $S g_{i}$ is simple and layered, so is in the $\mathbb{Z}\left[\boldsymbol{q}-\boldsymbol{q}^{-1}\right]$-span of $\mathcal{B}$, by Proposition 6.6. Moreover $(T-S) g_{i}$ is in the $\mathbb{Z}\left[z^{ \pm 1},\left(\boldsymbol{q}-\boldsymbol{q}^{-1}\right)\right]$-span of $\mathcal{B}$, by combining Lemma 6.5, Proposition 6.6, and the induction hypothesis.

The proof for right multiplication by $g_{i}^{-1}$ or by left multiplication by $g_{i}^{ \pm 1}$ is similar.

Remark 6.9 Let $T$ be a simple layered tangle diagram. From the proof of Lemma 6.5 and Proposition 6.6, one sees that all the elements of the Morton-Wassermann basis $\mathcal{B}$ that figure in the expansion of $T$ with respect to $\mathcal{B}$ are obtained by changing or smoothing various crossings of $T$. Hence, if $T$ has a strand $s$ connecting two vertices $v_{1}, v_{2}$, such that $s$ has no crossings with any other strand, then all elements of $\mathcal{B}$ appearing in the expansion of $T$ also have a strand connecting $v_{1}$ and $v_{2}$. Likewise, from the proof of Lemma 6.8, if $\{\overline{\boldsymbol{i}}, \overline{\boldsymbol{i}+\mathbf{1}}\} \cap\left\{v_{1}, v_{2}\right\}=\emptyset$, then all elements of $\mathcal{B}$ appearing in the expansion of $T g_{i}$ have a strand connecting $v_{1}$ and $v_{2}$.

\subsubsection{The Transition Matrix from a Tangle Basis to the Murphy Basis}

We examine the coefficients of the expansion of an element

$$
d_{\mathfrak{s}}^{*} c_{(\lambda, 0)} e_{n-1}^{(l)} d_{\mathfrak{t}}
$$

of $\mathscr{W}_{n}$ with respect to a Morton-Wassermann tangle basis $\mathcal{B}$ of $W_{n}\left(R_{0}\right)$.

Definition 6.10 Let $k \leq n$ and $m \leq\lfloor k / 2\rfloor$. A tangle diagram $T$ is of type $(k, m)$ if $T$ has strands connecting the adjacent pairs of bottom vertices

$$
(\overline{k-2 m+1}, \overline{k-2 m+2}), \ldots,(\overline{k-1}, \bar{k}) \quad(m \text { strands }) .
$$

Lemma 6.11 If $T$ is an element of $\mathcal{B}$ of type $(k, m)$, and $(\lambda, l) \rightarrow(\mu, m)$ is an edge in $\widehat{W}$ from level $k-1$ to level $k$, then $T d_{(\lambda, l) \rightarrow(\mu, m)}^{(k)}$ is a $\mathbb{Z}\left[\boldsymbol{q}^{ \pm 1}, z^{ \pm 1}\right]$-linear combination of elements of $\mathcal{B}$ of type $(k-1, l)$.

Proof There are two cases to consider.

CASE $1, l=m$ and $\lambda \subset \mu$. Then for some $a \leq k-2 m$, 


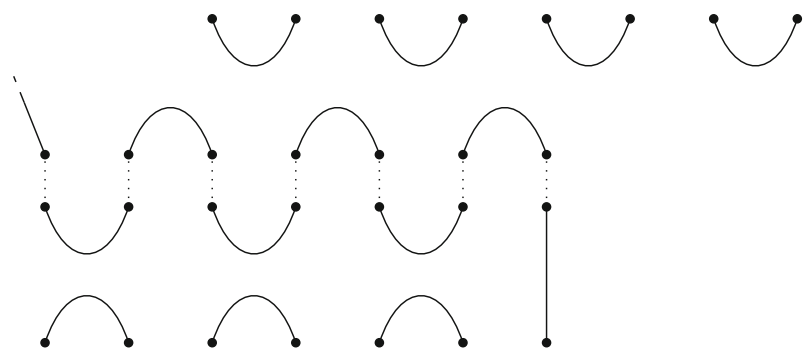

Fig. 1 Product $S e_{k-2}^{(m)}$, where $S$ is of type $(k, m)$

$$
d_{(\lambda, l) \rightarrow(\mu, m)}^{(k)}=g_{a, k-2 m} e_{k-2}^{(m)} .
$$

By Lemma 6.8 and Remark 6.9, $T g_{a, k-2 m}$ is a $\mathbb{Z}\left[\boldsymbol{q}^{ \pm 1}, \boldsymbol{z}^{ \pm 1}\right]$-linear combination of elements of $\mathcal{B}$ of type $(k, m)$. But for any element $S$ of $\mathcal{B}$ of type $(k, m), S e_{k-2}^{(m)}$ is a simple layered tangle diagram of type $(k-1, m)$, see Fig. 1. Therefore by Proposition 6.6 and Remark 6.9, $S e_{k-2}^{(m)}$ is a $\mathbb{Z}\left[\boldsymbol{q}-\boldsymbol{q}^{-1}\right]$-linear combination of elements of $\mathcal{B}$ of type $(k-1, m)$. Taking into account that $l=m$, this gives the result.

CASE $2, l=m-1$ and $\mu \subset \lambda$. Then $d_{(\lambda, l) \rightarrow(\mu, m)}^{(k)}$ is a sum of terms of the form $q^{s} g_{k+1-2 m, a} e_{k-2}^{(m-1)}$. But $T g_{k+1-2 m, a} e_{k-2}^{(m-1)}$ is a simple layered tangle diagram of type $(k-1, m-1)$, see Fig. 2. Therefore, again by Proposition 6.6 and Remark 6.9, $T g_{k+1-2 m, a} e_{k-2}^{(m-1)}$ is a $\mathbb{Z}\left[\boldsymbol{q}-\boldsymbol{q}^{-1}\right]$-linear combination of elements of $\mathcal{B}$ of type $(k-1, m-$ $1)$. Since $l=m-1$, this proves the result.

Proposition 6.12 $d_{\mathfrak{s}}^{*} c_{(\lambda, 0)} e_{n-1}^{(l)} d_{\mathfrak{t}}$ is in the $\mathbb{Z}\left[\boldsymbol{q}^{ \pm 1}, z^{ \pm 1}\right]$-span of $\mathcal{B}$.

Proof Taking into account Corollary 6.7, we can assume without loss of generality that the elements $g_{v} e_{n-1}^{(l)}$ for $v \in \mathfrak{S}_{n-2 f}$ are elements of $\mathcal{B}$, as these are simple layered tangle diagrams (with distinct underlying Brauer diagrams). Moreover, $g_{v} e_{n-1}^{(l)}$ is of type $(n, l)$. Thus $c_{(\lambda, 0)} e_{n-1}^{(l)}=\sum_{v \in \mathfrak{S}_{\lambda}} q^{l(v)} g_{v} e_{n-1}^{(l)}$ is in the $\mathbb{Z}[q]$-span of elements of $\mathcal{B}$ of type $(n, l)$.

Let

$$
\mathfrak{s}=\left(\left(\lambda^{(0)}, l_{0}\right),\left(\lambda^{(1)}, l_{1}\right), \ldots,\left(\lambda^{(n)}, l_{n}\right)\right),
$$

where $\left(\lambda^{(0)}, l_{0}\right)=(\emptyset, 0)$ and $\left(\lambda^{(n)}, l_{n}\right)=(\lambda, l)$. Then

$$
d_{\mathfrak{s}}=d_{\left(\lambda^{(n-1)}, l_{n-1}\right) \rightarrow\left(\lambda^{(n)}, l_{n}\right)}^{(n)} d_{\left(\lambda^{(n-2)}, l_{n-2}\right) \rightarrow\left(\lambda^{(n-1)}, l_{n-1}\right)}^{(n-1)} \cdots
$$

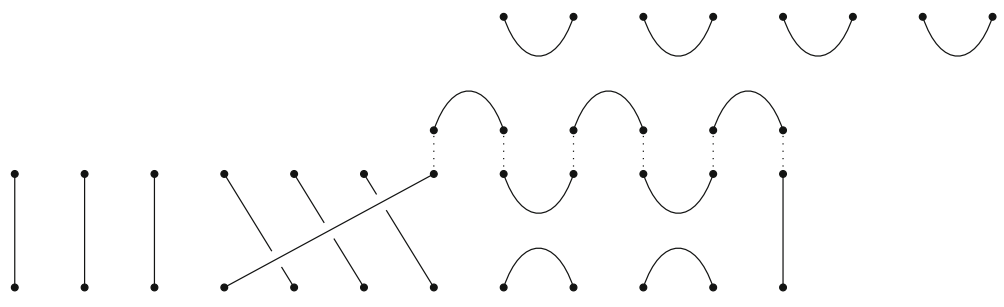

Fig. 2 Product $T g_{k+1-2 m, a} e_{k-2}^{(m-1)}$, where $T$ is of type $(k, m)$ 
By repeated use of Lemma $6.11, c_{(\lambda, 0)} e_{n-1}^{(l)} d_{\mathfrak{s}}$ is in the $\mathbb{Z}\left[\boldsymbol{q}^{ \pm 1}, z^{ \pm 1}\right]$-span of elements of $\mathcal{B}$. But the expansion of $\left(c_{(\lambda, 0)} e_{n-1}^{(l)} d_{\mathfrak{s}}\right)^{*}=d_{\mathfrak{s}}^{*} c_{(\lambda, 0)} e_{n-1}^{(l)}$ involves only elements of $\mathcal{B}$ of type $(n, l)$. By repeated application of Lemma 6.11 once more, $d_{\mathfrak{s}}^{*} c_{(\lambda, 0)} e_{n-1}^{(l)} d_{\mathfrak{t}}$ is in the $\mathbb{Z}\left[\boldsymbol{q}^{ \pm 1}, \boldsymbol{z}^{ \pm 1}\right]$-span of $\mathcal{B}$.

\subsubsection{The Murphy Basis and the Generic Ground Ring}

Let $B$ denote the matrix of expansion coefficients of the elements of $\mathscr{W}_{n}$ with respect to some Morton-Wassermann tangle basis $\mathcal{B}$ of $W_{n}\left(R_{0}\right)$ (and some choice of ordering of $\mathscr{W}_{n}$ and of $\mathcal{B}$ ). By Proposition 6.12, we know that the matrix $B$ has entries in $\mathbb{Z}\left[z^{ \pm 1}, \boldsymbol{q}^{ \pm 1}\right] \subset R_{0}$. On the other hand, since $\mathscr{W}_{n}$ is a basis of the BMW algebra over $R=R_{0}\left[\delta^{-1}\right]$, it follows that $B$ is invertible over $R$. We are going to show that $B$ is invertible over $\mathbb{Z}\left[z^{ \pm 1}, \boldsymbol{q}^{ \pm 1}\right]$ and therefore $\mathscr{W}_{n}$ is a basis of $W_{n}$ over $R_{0}$.

The Brauer algebra $B_{n}$ over $\mathbb{Z}[\delta]$ is the specialization of $W_{n}\left(R_{0}\right)$ at $q=1$ and $z=1$. (See the following Section 6.3 for details). Under the specialization, the Morton-Wassermann basis of $W_{n}\left(R_{0}\right)$ specializes to the usual diagram basis of the Brauer algebra, and $\mathscr{W}_{n}$ specializes to the corresponding collection of elements of the Brauer algebra, denoted $\mathscr{B}_{n}$. Moreover, the evaluation of $B$ at $q=1$ and $z=1$, which we denote by $B_{\mathbb{Z}}$, is the matrix of expansion coefficients of the elements of $\mathscr{B}_{n}$ with respect to the diagram basis of the Brauer algebra. Let $d$ denote the determinant of $B$ and $\bar{d}$ the determinant of $B_{\mathbb{Z}}$, which is the evaluation of $d$ at $q=1$ and $z=1$. Since $B$ is a matrix over $\mathbb{Z}\left[z^{ \pm 1}, \boldsymbol{q}^{ \pm 1}\right]$, it follows that $B_{\mathbb{Z}}$ is a matrix over $\mathbb{Z}$, and hence $\bar{d}$ is an integer.

Lemma 6.13 $B_{\mathbb{Z}}$ is invertible over $\mathbb{Z}$.

Proof Since $B$ is invertible over $R$, it follows that $B_{\mathbb{Z}}$ is invertible over $\mathbb{Z}\left[\delta^{ \pm 1}\right]$. Equivalently, $\bar{d}=\operatorname{det}\left(B_{\mathbb{Z}}\right)$ is a unit in $\mathbb{Z}\left[\delta^{ \pm 1}\right]$. But $\bar{d}$ is an integer, so it follows that $\bar{d}= \pm 1$ and thus $B_{\mathbb{Z}}$ is invertible over $\mathbb{Z}$.

Lemma 6.14 $B$ is invertible over $R_{0}$.

Proof Since $B$ is invertible over $R, d=\operatorname{det}(B)$ is a unit in $R$. We can regard $R$ as a subring of

$$
\bar{R}=\mathbb{Z}\left[z^{ \pm 1}, \boldsymbol{q}^{ \pm 1},(\boldsymbol{q}-1)^{-1},(\boldsymbol{q}+1)^{-1},(z+\boldsymbol{q})^{-1},(\boldsymbol{q} z-1)^{-1}\right],
$$

see Eq. (6.1). Since $d$ is an element of $\mathbb{Z}\left[z^{ \pm 1}, \boldsymbol{q}^{ \pm 1}\right] \subseteq R_{0}$ which is a unit in in $\bar{R}$, it has the form

$$
d= \pm \boldsymbol{q}^{a} z^{b}(\boldsymbol{q}-1)^{c}(\boldsymbol{q}+1)^{e}(z+\boldsymbol{q})^{f}(\boldsymbol{q} z-1)^{g}
$$

for some integers $a, b$ and some natural numbers $c, e, f, g$. But the specialization of $d$ at $q=1$ and $z=1$ is equal to \pm 1 and therefore we must have $c=e=f=g=0$. Thus $d= \pm \boldsymbol{q}^{a} z^{b}$ is a unit in $R_{0}$, so $B$ is invertible over $R_{0}$.

The invertibility of $B$ over $R_{0}$ together with Proposition 6.2 implies the following theorem:

Theorem 6.15 Let $W_{n}$ denote the BMW algebra over the generic ground ring $R_{0}$. The set

$$
\mathscr{W}_{n}=\left\{d_{\mathfrak{s}}^{*} c_{(\lambda, l)} d_{\mathfrak{t}} \mid \mathfrak{s}, \mathfrak{t} \in \widehat{W}_{n}^{(\lambda, l)},(\lambda, l) \in \widehat{W}_{n}\right\}
$$

is an $R_{0}$-basis of $W_{n}$, and $\left(W_{n}, *, \widehat{W}_{n}, \unrhd, \mathscr{W}_{n}\right)$ is a cell datum for $W_{n}$. 
Remark 6.16 The basis (6.3) differs from the Murphy-type basis for the BMW algebras given in [9] by a triangular transformation.

Corollary 6.17 For $n \geq 0$ and for $\Delta$ a cell module of $W_{n+1}$, the restricted module $\operatorname{Res}_{W_{n}}^{W_{n+1}}(\Delta)$ has an order preserving cell filtration.

Proof For $k \geq 0,(\lambda, l) \in \widehat{W}_{k}$, and $\mathfrak{t} \in \widehat{W}_{k}^{(\lambda, l)}$, let $m_{\mathfrak{t}}=\left(c_{(\lambda, l)}+W_{k}^{\triangleright(\lambda, l)}\right) d_{\mathfrak{t}}$. Then $\left\{m_{\mathfrak{t}}: \mathfrak{t} \in \widehat{W}_{k}^{(\lambda, l)}\right\}$ is the basis of the cell module $\Delta_{W_{k}}^{(\lambda, l)}$ derived from the cellular bases $\mathscr{W}$. The collection of these bases, as $k$ and $(\lambda, l)$ vary, is a family of path bases, because the path basis condition holds over $R=R_{0}\left[\delta^{-1}\right]$, according to Lemma 3.12, and therefore it holds over $R_{0}$ as well. It follows from Lemma 3.9 that restrictions of cell modules have an order preserving cell filtration.

\subsection{Brauer Algebras}

The Brauer algebras were defined by Brauer [4]. Wenzl [40] showed that the Brauer algebras are obtained from the group algebra of the symmetric group by the Jones basic construction, and that the Brauer algebras over a field of characteristic zero are generically semisimple. Cellularity of the Brauer algebras was established by Graham and Lehrer [16].

Let $S$ be an integral domain with a distinguished element $\delta$. The Brauer algebra $B_{n}=$ $B_{n}(S ; \delta)$ is the free $S$-module with basis the set of $(n, n)$-Brauer diagrams. The product of two Brauer diagrams is obtained by stacking them and then replacing each closed loop by a factor of $\delta$; see [4] or [40] for details.

Definition 6.18 Let $S$ be an integral domain and $\delta \in S$. The Brauer algebra $B_{n}=B_{n}(S ; \delta)$ is the free $S$-module with basis the set of $(n, n)$-Brauer diagrams, with bilinear product determined by the multiplication of Brauer diagrams. By convention, $B_{0}(S ; \delta)=S$.

The involution $*$ on $(n, n)$-Brauer diagrams which reflects a diagram in the axis $y=$ $1 / 2$ extends linearly to an algebra involution of $B_{n}(S ; \delta)$. Note that the Brauer diagrams with only vertical strands are in bijection with permutations of $\{1, \ldots, n\}$, and the multiplication of two such diagrams coincides with the multiplication of permutations. Thus the Brauer algebra contains the group algebra $S \mathfrak{S}_{n}$ of the permutation group $\mathfrak{S}_{n}$ as a unital subalgebra. The identity element of the Brauer algebra is the diagram corresponding to the trivial permutation. We will note below that $S \mathfrak{S}_{n}$ is also a quotient of $B_{n}(S ; \delta)$.

Let $s_{i}$ and $e_{i}$ denote the following $(n, n)$-Brauer diagrams:

$$
s_{i}=\lceil\ldots \downarrow
$$
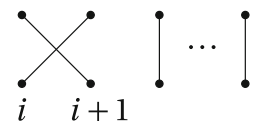

and
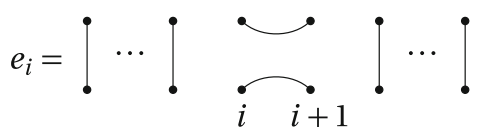

It is easy to see that $e_{1}, \ldots, e_{n-1}$ and $s_{1}, \ldots, s_{n-1}$ generate $B_{n}(S ; \delta)$ as an algebra. We have $e_{i}^{2}=\delta e_{i}$, so that $e_{i}$ is an essential idempotent if $\delta \neq 0$ and nilpotent otherwise. Note that $e_{i}^{*}=e_{i}$ and $s_{i}^{*}=s_{i}$.

The products $a b$ and $b a$ of two Brauer diagrams have at most as many through strands as $a$. Consequently, the span of diagrams with fewer than $n$ through strands is an ideal $J_{n}$ in $B_{n}(S ; \delta)$. The ideal $J_{n}$ is generated by $e_{n-1}$. We have $B_{n}(S ; \delta) / J_{n} \cong S \mathfrak{S}_{n}$, as 
algebras with involutions; in fact, the isomorphism is determined by $v+J_{n} \mapsto v$, for $v \in \mathfrak{S}_{n}$.

Morton and Wassermann show [31] that $B_{n}(S ; \delta)$ is a specialization of the BMW algebra $W_{n}(S ; q, z, \delta)$ at $q=1$ and $z=1$. Consequently, $B_{n}(S ; \delta)$ has a presentation by generators $s_{i}$ and $e_{i}(1 \leq i \leq n-1)$ and relations specializing those of the BMW algebra.

\subsubsection{The Murphy Basis}

The generic ground ring for the Brauer algebras is $R_{0}=\mathbb{Z}[\delta]$, where $\delta$ is an indeterminant. Write $R=\mathbb{Z}\left[\delta^{ \pm 1}\right]$, and write $B_{n}(R)=B_{n}(R ; \delta)$.

For $n \geq 0$ write $H_{n}=R \mathfrak{S}_{n}$. Specializing the cellular basis for $\mathcal{H}_{n}\left(q^{2}\right)$ given in Theorem 4.1 at $q=1$ gives a cellular basis for $H_{n}$. As for the Hecke algebras, $\widehat{H}_{n}$ is the set $\mathcal{Y}_{n}$ of Young diagrams of size $n$, and the branching diagram for the tower $\left(H_{n}\right)_{n \geq 0}$ of symmetric group algebras is Young's lattice.

It is shown in [13, Sect. 5.2] that the pair of towers $\left(B_{n}(R)\right)_{n \geq 0}$ and $\left(H_{n}\right)_{n \geq 0}$ satisfy the framework axioms (1)-(7) of Section 5.1. Axiom (8) holds by Corollary 4.10, and specialization from the Hecke algebras to the symmetric group algebras. Axiom (9) hold for $B_{n}(R)$, by the remarks at the end of Section 5.1. Moreover, by Corollary 4.3, the symmetric group algebras are cyclic cellular, so Axiom (10) is satisfied as well. Therefore, by Theorem 5.5, the tower of algebras $\left(B_{n}(R)\right)_{n \geq 0}$ is a strongly coherent tower of cyclic cellular algebras.

By the discussion in Section 5.4, the partially ordered set $\widehat{B}_{n}$ in the cell datum for $B_{n}$ can be realized as

$$
\widehat{B}_{n}=\left\{(\lambda, l) \mid 0 \leq l \leq\lfloor n / 2\rfloor \text { and } \lambda \in \widehat{H}_{n-2 l}\right\} .
$$

The order relation on $\widehat{B}_{n}$, and the branching rule for the branching diagram $\widehat{B}$ for the tower $\left(B_{n}\right)_{n>0}$ are exactly the same as for the BMW algebras discussed in the previous section.

For each $n \geq 0$ and for each $\mu \in \widehat{H}_{n}$, define $c_{(\mu, 0)}=\sum_{v \in \mathfrak{S}_{\mu}} v$; thus $c_{(\mu, 0)}$ is a preimage in $B_{n}$ of $m_{\mu} \in H_{n}$ (defined in Eq. (4.2)). For $n \geq 2$ and $(\mu, m) \in \widehat{B}_{n}$, let $c_{(\mu, m)}=$ $c_{(\mu, 0)} e_{n-1}^{(m)}$, where $e_{i-1}^{(m)}$ is defined in Eq. (5.4).

For $1 \leq i \leq j$ let

$$
s_{i, j}=s_{i} s_{i+1} \cdots s_{j-1}=(j, j-1, \ldots, i),
$$

and let $s_{j, i}=s_{i, j}^{-1}$.

Let $i \geq 1$ and $\lambda \in \widehat{\mathcal{H}}_{i-1}$ and $\mu \in \widehat{\mathcal{H}}_{i}$, with $\lambda \rightarrow \mu$ in $\widehat{\mathcal{H}}$. If $\mu=\lambda \cup\left\{\left(r, \mu_{r}\right)\right\}$, let $a=\sum_{j=1}^{r} \mu_{j}$, and define

$$
\bar{u}_{\lambda \rightarrow \mu}^{(i)}=s_{i, a} \sum_{k=0}^{\lambda_{r}} s_{a, a-k} \quad \text { and } \quad \bar{d}_{\lambda \rightarrow \mu}^{(i)}=s_{a, i} .
$$

These are liftings in $B_{i}$ of the branching factors in the symmetric group algebra $H_{i}$, as determined in Corollary 4.5 and Corollary 4.19.

For $(\lambda, l) \in \widehat{B}_{i}$ and $(\mu, m) \in \widehat{B}_{i+1}$ with $(\lambda, l) \rightarrow(\mu, m)$, determine the branching factors $d_{(\lambda, l) \rightarrow(\mu, m)}^{(i+1)}$ and $u_{(\lambda, l) \rightarrow(\mu, m)}^{(i+1)}$ according to the formulas of Theorem 5.7; for a path $\mathfrak{t} \in \widehat{W}_{n}^{(\lambda, l)}$, define $d_{\mathfrak{t}}$ to be the ordered product of these branching factors along the path $\mathfrak{t}$, as in Eq. (3.4). From Corollary 3.11 we obtain: 
Theorem 6.19 Let $B_{n}$ denote the Brauer algebra over the generic ground ring $R_{0}=\mathbb{Z}[\delta]$. For $n \geq 0$, the set

$$
\mathscr{B}_{n}=\left\{d_{\mathfrak{s}}^{*} c_{(\lambda, l)} d_{\mathfrak{t}} \mid \mathfrak{s}, \mathfrak{t} \in \widehat{B}_{n}^{(\lambda, l)},(\lambda, l) \in \widehat{B}_{n}\right\},
$$

is an $R_{0}$-basis for $B_{n}$, and $\left(B_{n}, *, \widehat{B}_{n}, \unrhd, \mathscr{B}_{n}\right)$ is a cell datum for $B_{n}$.

Proof Let $R=\mathbb{Z}\left[\delta^{ \pm 1}\right]$. From the preceding discussion and Corollary 3.11, we have that $\mathscr{B}_{n}$ is a cellular basis of $B_{n}(R ; \delta)$. In Section 6.2.4, we have shown that the transition matrix $B_{\mathbb{Z}}$ from the diagram basis of the Brauer algebra to $\mathscr{B}_{n}$ is integer valued and invertible over $\mathbb{Z}$. It follows that $\mathscr{B}_{n}$ is a basis of the Brauer algebra $B_{n}$ over the generic ground ring $R_{0}$.

Corollary 6.20 For $n \geq 0$ and for $\Delta$ a cell module of $B_{n+1}$, the restricted module

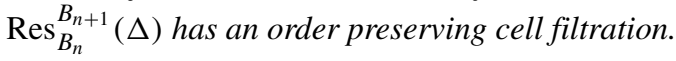

Proof The proof is the same as that of Corollary 5.17.

Remark 6.21 The basis (6.9) coincides with the Murphy-type basis for $B_{n}(\delta)$ given in [9].

\subsection{Jones-Temperley-Lieb Algebras}

The Jones-Temperley-Lieb algebras were defined by Jones [19], and were used to define the Jones link invariant in [21]. The cellularity of Jones-Temperley-Lieb algebras was established by Graham and Lehrer [16]. Härterich [18] has given Murphy bases for generalized Temperley-Lieb algebras.

Let $S$ be an integral domain and $\delta \in S$. The Jones-Temperley-Lieb algebra $A_{n}=$ $A_{n}(S ; \delta)$ is the unital $S$-algebra presented by the generators $e_{1}, \ldots, e_{n-1}$ and the relations $e_{i} e_{i \pm 1} e_{i}=e_{i}, e_{i} e_{j}=e_{j} e_{i}$ if $|i-j| \geq 2$, and $e_{i}^{2}=\delta e_{i}$. The Jones-Temperley-Lieb algebra can also be realized as the subalgebra of the Brauer algebra, with parameter $\delta$, spanned by Brauer diagrams without crossings. Because of the symmetry of the relations the assignment $e_{i} \mapsto e_{i}$ determines an involution $*$ of $A_{n}$. The span of diagrams with at least one horizontal strand (that is, all diagrams other than the identity diagram) is an ideal $J_{n}$; it is the ideal generated by $e_{n-1}$. The map $A_{n} / J_{n} \rightarrow S$ determined by $1_{A_{n}}+J_{n} \mapsto 1_{S}$ is an isomorphism of algebras with involution.

The generic ground ring for the Jones-Temperley-Lieb algebras is $R_{0}=\mathbb{Z}[\delta]$, where $\delta$ is an indeterminant over $\mathbb{Z}$. Set $R=\mathbb{Z}\left[\boldsymbol{\delta}^{ \pm 1}\right]$. Write $A_{n}(R)=A_{n}(R ; \boldsymbol{\delta})$, and $H_{n}=R$ for $n \geq 0$.

\subsubsection{The Murphy Basis}

It is shown in [13, Sect. 5.3] that the pair of towers $\left(A_{n}(R)\right)_{n \geq 0}$ and $\left(H_{n}\right)_{n \geq 0}$ satisfies the framework axioms (1)-(7) of Section 5.1. Axioms (8) and (10) are evident since $H_{n}=R$ for all $n$. Axiom (9) hold for $A_{n}(R)$, by the remarks at the end of Section 5.1. Therefore, by Theorem 5.5 , the tower of algebras $\left(A_{n}(R)\right)_{n \geq 0}$ is a strongly coherent tower of cyclic cellular algebras.

For each $n \geq 0$, the partially ordered set $\widehat{H}_{n}$ in the cell datum for $H_{n}$ is a singleton which we label as $\{n\}$, and the branching diagram for the tower $\left(H_{n}\right)_{n \geq 0}$ is $\emptyset=0 \rightarrow 1 \rightarrow 2 \rightarrow \cdots$. 
The branching diagram $\widehat{A}$ for the tower $\left(A_{n}\right)_{n \geq 0}$ is that obtained by reflections from $\widehat{H}$. It can be realized as follows: For $n \geq 0$, let

$$
\widehat{A}_{n}=\{j \mid 0 \leq j \leq n \text { and } n-j \text { is even }\}
$$

and order $\widehat{A}_{n}$ by writing $m \unrhd l$ if $l \geq m$ as integers. The branching diagram $\widehat{A}$ has an edge connecting $j$ on level $n$ and $k$ on level $n+1$ if and only if $|j-k|=1$.

Evidently, the algebra $H_{n}=R$ has the cellular basis $\{1\}$. We can choose the element $c_{n}$ in $H_{n}$ (see Lemma 2.9) to be 1 and also all the branching factors $d_{(n-1) \rightarrow n}^{(n)}$ and $u_{(n-1) \rightarrow n}^{(n)}$ to be 1 . According to Eq. (5.5), for $j \in \widehat{A}_{n}$, we can take

$$
c_{j}=e_{n-1}^{(l)}, \quad \text { where } l=(n-j) / 2,
$$

and $e_{n-1}^{(l)}$ is defined in Eq. (5.4). By Theorem 5.7, the branching factors for the tower $\left(A_{n}\right)_{n \geq 0}$ can be chosen as follows: If $j \in \widehat{A}_{i}$ and $k \in \widehat{A}_{i+1}$ with $j \rightarrow k$, we take

$$
d_{j \rightarrow k}^{(i+1)}=e_{i-1}^{(l)}, \quad \text { where } l=(i-j) / 2 .
$$

For a path $\mathfrak{t} \in \widehat{A}_{n}^{(\lambda, l)}$, define $d_{\mathfrak{t}}$ to be the ordered product of these branching factors along the path $\mathfrak{t}$, as in Eq. (3.4). From Corollary 3.11 we obtain:

Proposition 6.22 Let $R=\mathbb{Z}\left[\boldsymbol{\delta}^{ \pm 1}\right]$ and let $A_{n}(R)=A_{n}(R ; \delta)$ denote the Jones-TemperleyLieb algebra over $R$. For $n \geq 0$, the set

$$
\mathscr{A}_{n}=\left\{d_{\mathfrak{s}}^{*} c_{l} d_{\mathfrak{t}} \mid \mathfrak{s}, \mathfrak{t} \in \widehat{A}_{n}^{l} \text { and } l \in \widehat{A}_{n}\right\},
$$

is an $R$-basis for $A_{n}$, and $\left(A_{n}, *, \widehat{A}_{n}, \unrhd, \mathscr{A}_{n}\right)$ is a cell datum for $A_{n}$.

\subsubsection{The Murphy Basis Coincides with the Diagram Basis}

Next, we will show that the Murphy type cellular basis $\mathscr{A}_{n}$ of $A_{n}$ given in Proposition 6.22 actually coincides with the diagram basis, so is in particular a basis for the JonesTemperley-Lieb algebra over the generic ground ring $\mathbb{Z}[\boldsymbol{\delta}]$.

Let $S$ be an integral domain and $\delta \in S$. Let $k$ and $n$ be non-negative integers of the same parity. A $(k, n)$-Temperley-Lieb diagram is a planar diagram with $k$ upper vertices and $n$ lower vertices connected in pairs with no crossings. The product of a $(k, n)-$ TL diagram and an $(n, m)$-TL diagram is defined by the same rule as the product of two ordinary TL diagrams of the same size; the result is a power of $\delta$ times a $(k, m)-\mathrm{TL}$ diagram. The Temperley-Lieb category is category whose objects are non-negative integers; if $n-k$ is odd, then $\operatorname{Hom}(k, n)=0$, and if $n-k$ is even then $\operatorname{Hom}(k, n)$ is the free $S$-module on the basis of $(k, n)$-TL diagrams. Composition of morphisms is the bilinear extension of the product of diagrams described above. There is a map $*$ from $(k, n)$-TL diagrams to $(n, k)-\mathrm{TL}$ diagrams defined by reflection in a horizontal line. The linear extension of $*$ is a contravariant functor from the TL category to itself with $* \circ *=\mathrm{id}$. The rank of a $(m, n)-\mathrm{TL}$ diagram is the number of its vertical strands.

Fix $n \geq 0$. A TL $n$-dangle of rank $k$ is a $(k, n)$-TL diagram with $k$ vertical strands and $(n-k) / 2$ horizontal strands. Any $(n, n)-\mathrm{TL}$ diagram $T$ of rank $k$ can be written uniquely as $T=y^{*} x$, where $x$ and $y$ are $n$-dangles of rank $k$. A Dyck sequence of length $n$ and rank $k$ is a sequence $\left(a_{1}, \ldots, a_{n}\right)$ such that $a_{i} \in\{ \pm 1\}$, each partial sum $\sum_{i=1}^{j} a_{i}$ is non-negative, and $\sum_{i=1}^{n} a_{i}=k$. There is a bijection between Dyck sequences of length $n$ and rank $k$, and $n$-dangles of rank $k$, given as follows. Given a Dyck sequence $\left(a_{i}\right)$ of length $n$ and rank $k$, 
there is a unique $n$-dangle $x$ of rank $k$ with the following property: a vertex $\bar{j}$ is the right endpoint of a horizontal strand of $x$ if and only if $a_{j}=-1$. Conversely, given an $n$-dangle $x$ of rank $k$, label the right endpoint of each horizontal strand with -1 and all other bottom vertices with +1 . Then the resulting sequence of labels in $\{ \pm 1\}$, read from left to right, is a Dyck sequence of rank $k$. The two maps, from Dyck sequences to dangles and from dangles to Dyck sequences, are inverses.

There is a bijection between paths on the generic branching diagram for the TemperleyLieb algebras, of length $n$, from $\emptyset$ to $k$, and Dyck sequences of length $n$ and rank $k$. A path is given by a sequence $\left(0=b_{0}, 1=b_{1}, b_{2}, \ldots, k=b_{n}\right)$ with $b_{j}-b_{j-1}= \pm 1$ for each $j$. Then the sequence $\left(b_{i}-b_{i-1}\right)_{i=1}^{n}$ is a Dyck sequence of length $n$ and rank $k$. Conversely, given a Dyck sequence of length $n$ and rank $k$, its sequence of partial sums defines a path on the branching diagram, of length $n$, from $\emptyset$ to $k$. Evidently, the two maps, from paths to Dyck sequences and from Dyck sequence to paths, are inverses.

Composing the two bijections described above, we have a bijection between paths on the branching diagram and dangles. For a path $\mathfrak{t}$ on the branching diagram, let $x(\mathfrak{t})$ denote the corresponding dangle.

Theorem 6.23 Fix $n$ and $k \leq n$ with $n-k$ even. Let $\mathfrak{s}$ and $\mathfrak{t}$ be elements of $\widehat{A_{n}^{k}}$. Then

$$
d_{\mathfrak{s}}^{*} c_{k} d_{\mathfrak{t}}=x(\mathfrak{s})^{*} x(\mathfrak{t})
$$

Thus the Murphy type basis

$$
\mathscr{A}_{n}=\left\{d_{\mathfrak{s}}^{*} c_{k} d_{\mathfrak{t}} \mid \mathfrak{s}, \mathfrak{t} \in \widehat{A}_{n}^{k} \text { and } k \in \widehat{A}_{n}\right\},
$$

is just the set of all Temperley-Lieb diagrams on $2 n$ vertices, and in particular is a cellular basis of the Jones-Temperley-Lieb algebra $A_{n}$ over the generic ground ring $\mathbb{Z}[\delta]$.

Proof Recall that $c_{k}=e_{n-1}^{(l)}$, where $l=(n-k) / 2$. Let $x_{n-1}^{(l)}$ be the bottom half of $e_{n-1}^{(l)}$, namely the $n$-dangle of rank $k$ with horizontal strands connecting the adjacent pairs of vertices

$$
(\overline{\boldsymbol{k}+\mathbf{1}}, \overline{\boldsymbol{k}+\mathbf{2}}), \ldots,(\overline{\boldsymbol{n}-\mathbf{1}}, \overline{\boldsymbol{n}}) \quad(l \text { strands }) .
$$

Thus $e_{n-1}^{(l)}=\left(x_{n-1}^{(l)}\right)^{*} x_{n-1}^{(l)}$. To prove the proposition it suffices to show that

$$
x_{n-1}^{(l)} d_{\mathfrak{t}}=x(\mathfrak{t}) .
$$

We do this by induction on $n$, the case $n=1$ being evident. Assume that the assertion holds for some fixed $n$, for all $k$ with $k \leq n$ and $n-k$ even, and for all $\mathfrak{t} \in \widehat{A_{n}^{k}}$. Let $\mathfrak{s} \in \widehat{A}_{n+1}^{j}$ for some $j$,

$$
\mathfrak{s}=\left(k_{0}, k_{1}, \ldots, k_{n}=k, k_{n+1}=j\right),
$$

and let $\mathfrak{t}$ be the truncation of $\mathfrak{s}$ of length $n$,

$$
\mathfrak{t}=\left(k_{0}, k_{1}, \ldots, k_{n}=k\right) .
$$

Write $l=(n-k) / 2$ and $l^{\prime}=(n+1-j) / 2$. There are two cases: 
Case 1. $j=k+1, l^{\prime}=l$. In this case, $x(\mathfrak{s})$ is obtained from $x(\mathfrak{t})$ by adding a vertical strand at the new vertex $\overline{\boldsymbol{n}+\mathbf{1}}$. On the other hand,

$$
\begin{aligned}
x_{n}^{\left(l^{\prime}\right)} d_{\mathfrak{s}} & =x_{n}^{(l)} d_{k \rightarrow k+1}^{(n+1)} d_{t} \\
& =x_{n}^{(l)} e_{n-1}^{(l)} d_{t} \\
& =x_{n}^{(l)}\left(x_{n-1}^{(l)}\right)^{*} x_{n-1}^{(l)} d_{t} \\
& =x_{n}^{(l)}\left(x_{n-1}^{(l)}\right)^{*} x(\mathfrak{t}),
\end{aligned}
$$

using the induction hypothesis at the last step. Multiplication of an $n$-dangle of rank $k$ on the left by $x_{n}^{(l)}\left(x_{n-1}^{(l)}\right)^{*}$ adds a vertical strand on the right, as shown in Fig. 3. Hence we have $x_{n}^{\left(l^{\prime}\right)} d_{\mathfrak{s}}=x(\mathfrak{s})$.

Case 2. $\quad j=k-1, l^{\prime}=l+1$. In this case, $x(s)$ is obtained from $x(t)$ by "closing" the rightmost vertical strand; that is, if $\bar{j}$ is the vertex adjacent to this strand, the strand is replaced by a horizontal strand joining $\bar{j}$ and $\overline{\boldsymbol{n}+\mathbf{1}}$. On the other hand,

$$
\begin{aligned}
x_{n}^{\left(l^{\prime}\right)} d_{\mathfrak{s}} & =x_{n}^{(l+1)} d_{k \rightarrow k+1}^{(n+1)} d_{t} \\
& =x_{n}^{(l+1)}\left(x_{n-1}^{(l)}\right)^{*} x(\mathfrak{t}),
\end{aligned}
$$

by the same computation as in the previous case. But multiplication of an $n$-dangle of rank $k$ on the left by $x_{n}^{(l+1)}\left(x_{n-1}^{(l)}\right)^{*}$ closes the rightmost vertical strand, as shown in Fig. 4. So again we have $x_{n}^{\left(l^{\prime}\right)} d_{\mathfrak{s}}=x(\mathfrak{s})$, and this completes the inductive proof.

Corollary 6.24 Let $A_{n}$ denote the Jones-Temperley-Lieb algebra over the generic ground ring $\mathbb{Z}[\delta]$. For $n \geq 0$ and for $\Delta$ a cell module of $A_{n+1}$, the restricted module $\operatorname{Res}_{A_{n}}^{A_{n+1}(\Delta)}$ has an order preserving cell filtration.

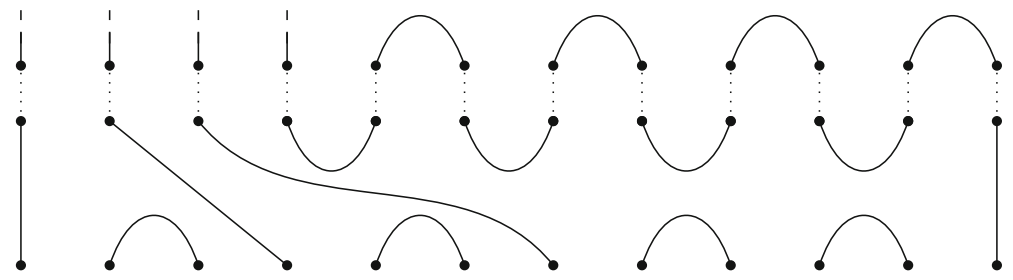

Fig. $3 x_{n}^{(l)}\left(x_{n-1}^{(l)}\right)^{*} x(\mathfrak{t})$

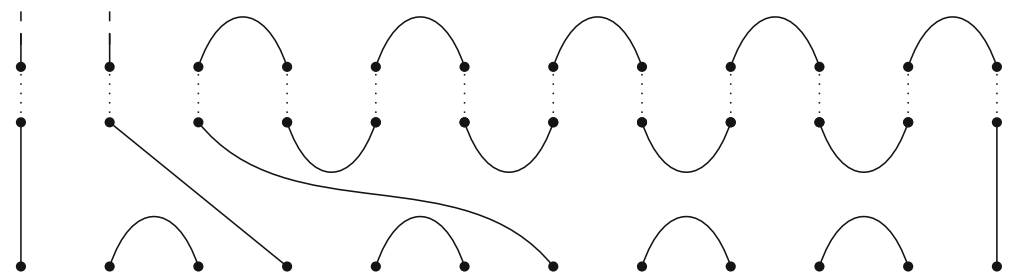

Fig. $4 x_{n}^{(l+1)}\left(x_{n-1}^{(l)}\right)^{*} x(\mathfrak{t})$ 
Proof One can either use the same proof as for Corollary 6.17, or one can check directly using a diagrammatic model of the cell modules that $\operatorname{Res}_{A_{n}}^{A_{n+1}}\left(\Delta_{A_{n+1}}^{k}\right)$ has a filtration

$$
0 \subseteq N \subseteq \operatorname{Res}_{A_{n}}^{A_{n+1}\left(\Delta_{A_{n+1}}^{k}\right),}
$$

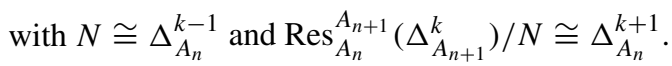

\subsection{Partition Algebras}

The partition algebras $A_{n}(k)$, for $k, n \in \mathbb{Z}_{\geq 0}$, are a family of algebras defined in the work of Martin and Jones in [20, 25-27] in connection with the Potts model and higher dimensional statistical mechanics. Jones [20] showed that the even partition algebra $A_{2 n}(k)$ is in SchurWeyl duality with the symmetric group $\mathfrak{S}_{k}$ acting diagonally on the $n$-fold tensor product $V^{\otimes n}$ of its $k$-dimensional permutation representation $V$. In [25], Martin defined the odd partition algebra $A_{2 n+1}(k)$ as the centralizer of the subgroup $\mathfrak{S}_{k-1} \subseteq \mathfrak{S}_{k}$ acting on $V^{\otimes n}$. Including the algebras $A_{2 n+1}(k)$ in the tower

$$
A_{0}(k) \subseteq A_{1}(k) \subseteq A_{2}(k) \subseteq A_{3}(k) \subseteq \cdots
$$

allowed for the simultaneous analysis of the whole tower of algebras (6.11) using the Jones basic construction, by Martin [25] and Halverson and Ram [17]. Cellularity of the partition algebras was proved in $[6,41,42]$.

For $n \in \mathbb{Z}_{\geq 0}$ let

$$
\begin{aligned}
P_{2 n} & =\{\text { set partitions of }\{\mathbf{1}, \mathbf{2}, \ldots, \boldsymbol{n}, \overline{\mathbf{1}}, \overline{\mathbf{2}}, \ldots, \overline{\boldsymbol{n}}\}\}, \quad \text { and, } \\
P_{2 n-1} & =\left\{d \in P_{2 n} \mid \boldsymbol{n} \text { and } \overline{\boldsymbol{n}} \text { are in the same block of } d\right\} .
\end{aligned}
$$

Any element $\rho \in P_{2 n}$ may be represented as a graph with $n$ vertices in the top row, labelled from left to right, by $\mathbf{1}, \mathbf{2}, \ldots, \boldsymbol{n}$ and $n$ vertices in the bottom row, labelled, from left to right by $\overline{\mathbf{1}}, \overline{\mathbf{2}}, \ldots, \overline{\boldsymbol{n}}$, with the connected components of the graph being the blocks of $\rho$. The representation of a partition by a diagram is not unique; for example the partition

$$
\rho=\{\{\mathbf{1}, \overline{\mathbf{1}}, \mathbf{3}, \overline{\mathbf{4}}, \overline{\mathbf{5}}, \mathbf{6}\},\{\mathbf{2}, \overline{\mathbf{2}}, \overline{\mathbf{3}}, \mathbf{4}, \mathbf{5}, \overline{\mathbf{6}}\}\}
$$

can be represented by the diagrams:

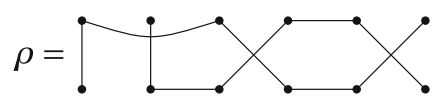

and

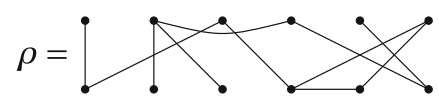

If $\rho_{1}, \rho_{2} \in P_{2 n}$, then the composition $\rho_{1} \circ \rho_{2}$ is the partition obtained by placing $\rho_{1}$ above $\rho_{2}$ and identifying each vertex in the bottom row of $\rho_{1}$ with the corresponding vertex in the top row of $\rho_{2}$ and deleting any components of the resulting diagram which contains only elements from the middle row.

Definition 6.25 Let $S$ be a commutative unital ring and $\delta \in S$. For $n \geq 1$, the partition algebra $A_{2 n}(S ; \delta)$ is the free $S$-module with basis $P_{2 n}$, equipped with the product $\rho_{1} \rho_{2}=$ $\delta^{l} \rho_{1} \circ \rho_{2}$, for $\rho_{1}, \rho_{2} \in P_{2 n}$, where $l$ is the number of blocks removed from the middle row in constructing the composition $\rho_{1} \circ \rho_{2}$. By convention, $A_{0}(S ; \delta)=S$. Let $A_{2 n-1}(S ; \delta)$ denote the subalgebra of $A_{2 n}(S ; \delta)$ spanned by $P_{2 n-1}$.

The Brauer algebra $B_{n}(S ; \delta)$ embeds as a subalgebra of $A_{2 n}(S ; \delta)$, spanned by partitions with each block having two elements. In particular, $A_{2 n}(S ; \delta)$ has a subalgebra isomorphic 
to the symmetric group algebra $S \mathfrak{S}_{n}$, spanned by permutation diagrams. The permutation subalgebra is generated by the transpositions

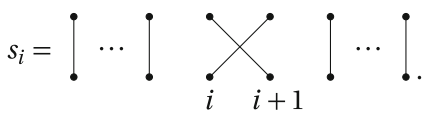

The multiplicative identity of $A_{2 n}(S ; \delta)$ is the trivial permutation. It is not hard to see that the partition algebra $A_{2 n}(S ; \delta)$ is generated by the transpositions $s_{i}(1 \leq i \leq n-1)$ and elements $e_{j}(1 \leq j \leq 2 n-1)$, where

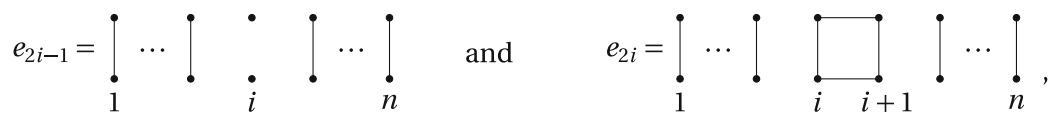

Halverson and Ram [17, Theorem 1.11] and East [7, Theorem 36] give a presentation for $A_{2 n}$ in terms of the generators $e_{j}$ and $s_{i}$. The algebras $A_{2 n}(S ; \delta)$ and $A_{2 n-1}(\delta)$ have an algebra involution $*$ which acts on diagrams by flipping them over the horizontal line $y=$ $1 / 2$. The generators $s_{i}$ and $e_{j}$ are $*$-invariant

$A_{2 n-1}(S ; \delta)$ is defined as a subalgebra of $A_{2 n}(S ; \delta)$, and $A_{2 n}(S ; \delta)$ embeds in $A_{2 n+1}(S ; \delta)$ as follows: define a map $\iota: P_{2 n} \rightarrow P_{2 n+1}$ by adding an additional block $\{\boldsymbol{n}+\mathbf{1}, \overline{\boldsymbol{n}+\mathbf{1}}\}$. The linear extension of $\iota$ is a monomorphism of algebras with involution.

Let $d \in P_{2 n}$. Call a block of $d$ a through block if the block has non-empty intersection with both $[\boldsymbol{n}]$ and $[\boldsymbol{n}]$. The number of through blocks of $d$ is called the propagating number of $d$, denoted $\operatorname{pn}(d)$. Clearly, $\operatorname{pn}(d) \leq n$ for all $d \in P_{2 n}$. The only $d \in P_{2 n}$ with propagating number equal to $n$ are the permutation diagrams. If $x, y \in P_{2 n}$ and $x y=\delta^{r} z$, then $\operatorname{pn}(z) \leq$ $\min \{\operatorname{pn}(x), \operatorname{pn}(y)\}$. Hence the span of the set of $d \in P_{2 n}$ with $\operatorname{pn}(d)<n$ is an ideal $J_{2 n} \subset$ $A_{2 n}(S ; \delta)$. Moreover, $J_{2 n-1}:=J_{2 n} \cap A_{2 n-1}$ is the span of $d \in P_{2 n-1}$ with pn $(d)<n$. One can check that for $k \geq 2, J_{k}$ is the ideal of $A_{k}(S ; \delta)$ generated by $e_{k-1}$. The ideal $J_{k}$ is $*-$ invariant, and the span of permutation diagrams in $A_{k}$ is a $*$-invariant linear complement for $J_{k}$. It follows that $A_{2 n}(S ; \delta) / J_{2 n} \cong S \mathfrak{S}_{n}$ and $A_{2 n-1}(S ; \delta) / J_{2 n-1} \cong S \mathfrak{S}_{n-1}$ as algebras with involution; the isomorphisms are determined by $v+J_{k} \mapsto v$, where $v$ is a permutation diagram.

\subsubsection{The Murphy Basis}

The generic ground ring for the partition algebras is $R_{0}=\mathbb{Z}[\delta]$, where $\delta$ is an indeterminant. Write $R=\mathbb{Z}\left[\boldsymbol{\delta}^{ \pm 1}\right]$, and let $F=\mathbb{Q}(\boldsymbol{\delta})$ denote the field of fractions of $R$. Write $A_{n}$ for $A_{n}(R ; \delta)$ and write $H_{2 i}=H_{2 i+1}=R \mathfrak{S}_{i}$ for $i \geq 0$. The tower $\left(H_{n}\right)_{n \geq 0}$ is a strongly coherent tower of cyclic cellular algebras, and $H_{n}^{F}$ is split semisimple. The branching diagram of the tower $\left(H_{n}\right)_{n \geq 0}$ is the graph $\widehat{H}$ with

(1) $\widehat{H}_{2 i}=\widehat{H}_{2 i+1}=$ the set $\mathcal{Y}_{i}$ of Young diagrams of size $i$.

(2) an edge $\lambda \rightarrow \mu$ in $\widehat{H}$ if

(a) $\lambda \in \widehat{H}_{2 i-1}, \mu \in \widehat{H}_{2 i}$ and $\lambda \subseteq \mu$, or

(b) $\lambda \in \widehat{H}_{2 i}, \mu \in \widehat{H}_{2 i+1}$ and $\lambda=\mu$.

It is shown in [13, Sect. 5.7] that the pair of towers $\left(A_{n}\right)_{n \geq 0}$ and $\left(H_{n}\right)_{n \geq 0}$ satisfy the framework axioms (1)-(7) of Section 5.1. Axiom (8) holds by Corollary 4.10. Axiom (9) holds for the partition algebras, by the remarks at the end of Section 5.1. Finally, Axiom (10) holds by Corollary 4.3. Therefore, by Theorem 5.5, the tower of algebras $\left(A_{n}\right)_{n \geq 0}$ is a strongly coherent tower of cyclic cellular algebras. 


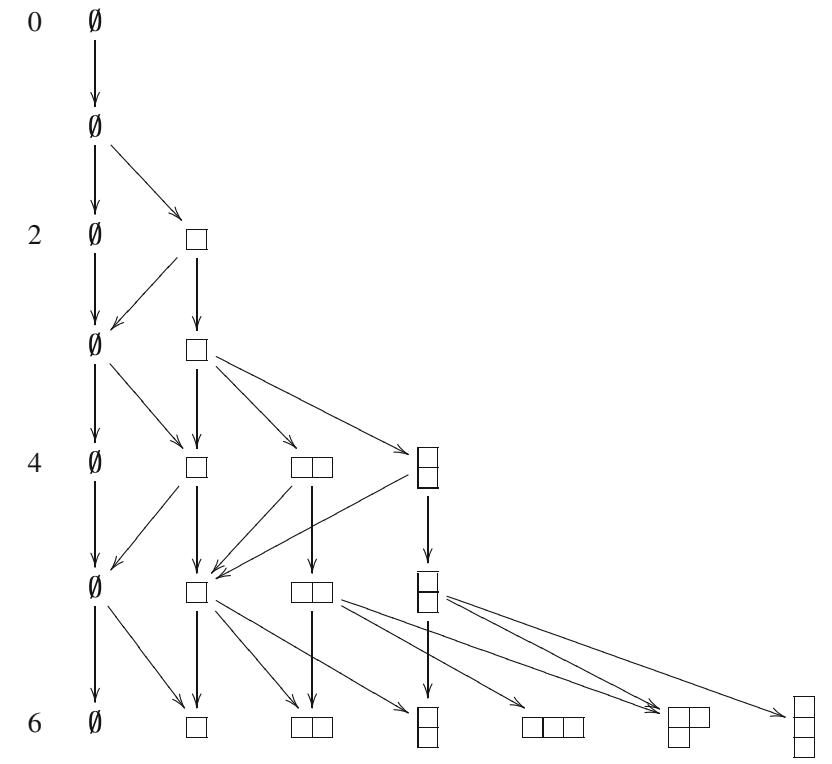

Fig. 5 Branching diagram for the partition algebras.

By the discussion in Section 5.4, the partially ordered set $\widehat{A}_{i}$ in the cell datum for $A_{i}$ can be realized as

$$
\widehat{A_{i}}=\left\{(\lambda, l) \mid \lambda \in \widehat{H}_{i-2 l}, \text { for } l=0,1, \ldots,\lfloor i / 2\rfloor\right\}
$$

ordered by $(\lambda, l) \unrhd(\mu, m)$ if $l>m$, or if $l=m$ and $\lambda \unrhd \mu$ as elements of $\widehat{H}_{i-2 l}$. The branching diagram $\widehat{A}$ for the tower $\left(A_{n}\right)_{n \geq 0}$ is that obtained by reflections from the branching diagram $\widehat{H}$. Thus the branching rule is the following:

(1) Let $i$ be even and $(\lambda, l) \in \widehat{A}_{i}$.

(a) For $(\mu, l) \in \widehat{A}_{i+1},(\lambda, l) \rightarrow(\mu, l)$ in $\widehat{A}$ if and only if $\lambda=\mu$.

(b) For $(\mu, l+1) \in \widehat{A}_{i+1},(\lambda, l) \rightarrow(\mu, l+1)$ in $\widehat{A}$ if and only $\mu \subset \lambda$.

(2) Let $i$ be odd and $(\lambda, l) \in \widehat{A_{i}}$.

(a) For $(\mu, l) \in \widehat{A}_{i+1},(\lambda, l) \rightarrow(\mu, l)$ in $\widehat{A}$ if and only if $\lambda \subset \mu$.

(b) For $(\mu, l+1) \in \widehat{A}_{i+1},(\lambda, l) \rightarrow(\mu, l+1)$ in $\widehat{A}$ if and only $\lambda=\mu$.

The first few levels of $\widehat{A}$ are given in Fig. 5 .

Next, we determine the branching coefficients for the two towers $\left(H_{n}\right)_{n \geq 0}$ and $\left(A_{n}\right)_{n \geq 0}$. Let $\lambda \in \widehat{H}_{2 i-1}$ and $\mu \in \widehat{H}_{2 i}$ with $\lambda \rightarrow \mu$ in $\widehat{H}$. If $\mu=\lambda \cup\left\{\left(r, \mu_{r}\right)\right\}$, let $a=\sum_{j=1}^{r} \mu_{j}$. Then the branching factors for the inclusion $H_{2 i-1} \subseteq H_{2 i}$ in the tower $\left(H_{i}\right)_{i \geq 0}$ are given by

$$
d_{\lambda \rightarrow \mu}^{(2 i)}=s_{a, i} \quad \text { and } \quad u_{\lambda \rightarrow \mu}^{(2 i)}=s_{i, a} \sum_{k=0}^{\lambda_{r}} s_{a, a-k},
$$

where the elements $s_{i, j}$ are defined in Eq. (6.7). The branching factors for the inclusion $H_{2 i} \subseteq H_{2 i+1}$ in the tower $\left(H_{i}\right)_{i \geq 0}$ are given by

$$
d_{\lambda \rightarrow \lambda}^{(2 i+1)}=u_{\lambda \rightarrow \lambda}^{(2 i+1)}=1 \quad \text { if } \lambda \in \widehat{H}_{2 i}=\widehat{H}_{2 i+1} .
$$


For $\lambda \in \widehat{H}_{k}$ and $\mu \in \widehat{H}_{k+1}$, define $\bar{d}_{\lambda \rightarrow \mu}^{(k+1)}$ and $\bar{u}_{\lambda \rightarrow \mu}^{(k+1)}$ by the same formulas, specifying elements of the subalgebra of $A_{k+1}$ spanned by permutation diagrams; these are liftings in $A_{k+1}$ of the branching factors in $H_{k+1}$ specified above.

By Theorem 5.7, the branching factors for the tower $\left(A_{n}\right)_{n \geq 0}$ can be chosen as follows: Let $(\lambda, l) \in \widehat{A}_{2 i-1}$. If $(\mu, l) \in \widehat{A}_{2 i}$ and $(\lambda, l) \rightarrow(\mu, l)$ in $\widehat{A}$, then $\lambda \subset \mu$ and

$$
d_{(\lambda, l) \rightarrow(\mu, l)}^{(2 i)}=\bar{d}_{\lambda \rightarrow \mu}^{(2 i-2 l)} e_{2 i-2}^{(l)},
$$

and, if $(\mu, l+1) \in \widehat{A}_{2 i}$ and $(\lambda, l) \rightarrow(\mu, l+1)$ in $\widehat{A}$, then $\lambda=\mu$ and

$$
d_{(\lambda, l) \rightarrow(\lambda, l+1)}^{(2 i)}=e_{2 i-2}^{(l)} .
$$

Similarly, if $(\lambda, l) \in \widehat{A}_{2 i}$ and $(\mu, l) \in \widehat{A}_{2 i+1}$ and $(\lambda, l) \rightarrow(\mu, l)$, then $\lambda=\mu$ and

$$
d_{(\lambda, l) \rightarrow(\lambda, l)}^{(2 i+1)}=e_{2 i-1}^{(l)},
$$

and, if $(\mu, l+1) \in \widehat{A}_{2 i+1}$ and $(\lambda, l) \rightarrow(\mu, l+1)$, then $\mu \subset \lambda$ and

$$
d_{(\lambda, l) \rightarrow(\mu, l+1)}^{(2 i+1)}=\bar{u}_{\mu \rightarrow \lambda}^{(2 i-2 l)} e_{2 i-1}^{(l)} .
$$

The $u$-coefficients $u_{(\lambda, l) \rightarrow \mu, m)}^{(n+1)}$ are determined by similar formulas by Theorem 5.7.

Fix $n \geq 1$ and $(\lambda, l) \in \widehat{A}_{n}$. For a path $\mathrm{t}$ in $\widehat{A}_{n}^{(\lambda, l)}$, define $d_{\mathfrak{t}}$ to be the ordered product of the $d$-branching coefficients for the tower $\left(A_{k}\right)$ along the path $\mathfrak{t}$, as in Eq. (3.4). Define $c_{(\lambda, l)}=$ $c_{(\lambda, 0)} e_{i-1}^{(l)}$, where $c_{(\lambda, 0)}=\sum_{v \in \mathfrak{S}_{\lambda}} v$, and $e_{i-1}^{(l)}$ is defined in Eq. (5.4). From Corollary 3.11 we obtain:

Proposition 6.26 Let $R=\mathbb{Z}\left[\delta^{ \pm 1}\right]$ and let $A_{n}=A_{n}(R ; \delta)$ be partition algebra defined over $R$ with parameter $\delta$. For each $n$, the set

$$
\mathscr{A}_{n}=\left\{d_{\mathfrak{s}}^{*} c_{(\lambda, l)} d_{\mathfrak{t}} \mid \mathfrak{s}, \mathfrak{t} \in \widehat{A}_{n}^{(\lambda, l)},(\lambda, l) \in \widehat{A}_{n}\right\},
$$

is an $R$-basis for $A_{n}$, and $\left(A_{n}, *, \widehat{A}_{n}, \unrhd, \mathscr{A}_{n}\right)$ is a cell datum for $A_{n}$.

\subsubsection{The Murphy Basis and the Generic Ground Ring}

It remains to show that the set $\mathscr{A}_{n}$ is a basis for the partition algebra $A_{n}\left(R_{0} ; \delta\right)$ defined over the generic ground ring $R_{0}=\mathbb{Z}[\delta]$. Let $\mathcal{B}$ denote the diagram basis for $A_{n}\left(R_{0} ; \delta\right)$.

Definition 6.27 Let $1 \leq l \leq k \leq j$. A set partition $\varrho$ of $P=\{\mathbf{1}, \ldots, \boldsymbol{j}, \overline{\mathbf{1}}, \ldots, \overline{\boldsymbol{j}}\}$ is said to be of even type $(k, l)$ if each element of the set of lower vertices $\{\overline{\boldsymbol{k}-\boldsymbol{l}+\mathbf{1}}, \overline{\boldsymbol{k}-\boldsymbol{l}+\mathbf{2}}, \ldots, \overline{\boldsymbol{k}}\}$ lives in a block of size one; $\varrho$ is said to be of odd type $(k, l)$ if all the lower vertices in the set $\{\overline{\boldsymbol{k}-\boldsymbol{l}}, \overline{\boldsymbol{k}-\boldsymbol{l}+\mathbf{1}}, \ldots, \overline{\boldsymbol{k}}\}$ live in the same block of $\varrho$.

Lemma 6.28 Let $\varrho$ be a partition.

(1) If $\varrho$ is of odd type $(k+1, m)$ and $(\lambda, l) \rightarrow(\mu, m)$ is an edge from level $2 k$ to level $2 k+1$ in $\widehat{A}$, then $\varrho d_{(\lambda, l) \rightarrow(\mu, m)}^{(2 k+1)}$ is a $\mathbb{Z}$-linear combination of partitions of even type $(k, l)$.

(2) If $\varrho$ is of even type $(k, m)$ and $(\lambda, l) \rightarrow(\mu, m)$ is an edge from level $2 k-1$ to level $2 k$ in $\widehat{A}$, then $\varrho d_{(\lambda, l) \rightarrow(\mu, m)}^{(2 k)}$ is a $\mathbb{Z}$-linear combination of partitions of odd type $(k, l)$.

Proof Assume that $\varrho$ is of odd type $(k+1, m)$ and $(\lambda, l) \rightarrow(\mu, m)$ is an edge from level $2 k$ to level $2 k+1$ in $\widehat{A}$. Thus $\varrho$ has lower vertices $\overline{\boldsymbol{k}-\boldsymbol{m}+\mathbf{1}}, \ldots, \overline{\boldsymbol{k}+\mathbf{1}}$ in one block. If $l=m$, 


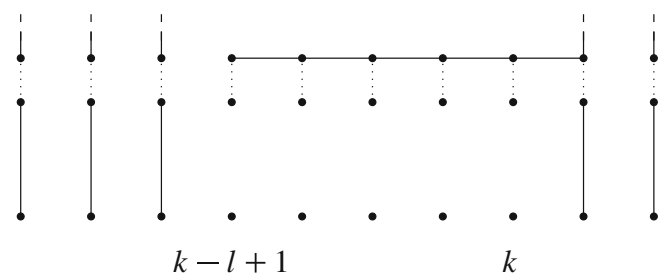

Fig. $6 \varrho e_{2 k-1}^{(l)}$, where $\varrho$ is of odd type $(k+1, l)$

then $\lambda=\mu$, and $d_{(\lambda, l) \rightarrow(\mu, l)}^{(2 k+1)}=e_{2 k-1}^{(l)}$. It follows that $\varrho d_{(\lambda, l) \rightarrow(\mu, m)}^{(2 k+1)}=\varrho e_{2 k-1}^{(l)}$ is equal to a single partition of even type $(k, l)$, and that no factor of $\delta$ arises in the computation of the product, as shown in Fig. 6. If $m=l+1$, then $\mu \subset l$ and

$$
d_{(\lambda, l) \rightarrow(\mu, l+1)}^{(2 k+1)}=\bar{u}_{\mu \rightarrow \lambda}^{(2 k-2 l)} e_{2 k-1}^{(l)},
$$

which is a sum of elements of the form $s_{k-l, j} e_{2 k-1}^{(l)}$ with $j \leq k-l$. It follows that $\varrho d_{(\lambda, l) \rightarrow(\mu, l+1)}^{(2 k+1)}$ is equal to a sum of distinct partitions, each of even type $(k, l)$, and again no factor of $\boldsymbol{\delta}$ appears in the computation of the product, as shown in Fig. 7.

Assume now that $\varrho$ is of even type $(k, m)$ and $(\lambda, l) \rightarrow(\mu, m)$ is an edge from level $2 k-1$ to level $2 k$ in $\widehat{A}$. Thus the lower vertices $\overline{\boldsymbol{k}-\boldsymbol{m}+\mathbf{1}}, \ldots, \overline{\boldsymbol{k}}$ each constitute a block of $\varrho$. If $l=m$, then $\lambda \subset \mu$ and

$$
d_{(\lambda, l) \rightarrow(\mu, l)}^{(2 k)}=\bar{d}_{\lambda \rightarrow \mu}^{(2 k-2 l)} e_{2 k-2}^{(l)} .
$$

But $\bar{d}_{\lambda \rightarrow \mu}^{(2 k-2 l)}=s_{j, k-l}$ for some $j \leq k-l$, and $\varrho^{\prime}=\varrho s_{j, k-l}$ is also a partition of even type $(k, l)$. Thus, we have to consider $\varrho d_{(\lambda, l) \rightarrow(\mu, l)}^{(2 k+1)}=\varrho s_{j, k-l} e_{2 k-2}^{(l)}=\varrho^{\prime} e_{2 k-2}^{(l)}$, where $\varrho^{\prime}$ is a partition of even type $(k, l)$. The product $\varrho^{\prime} e_{2 k-2}^{(l)}$ is a single partition, of odd type $(k, l)$, and no power of $\delta$ occurs in the computation of the product, as shown in Fig. 8.

Finally, if $m=l+1$, then $\lambda=\mu$ and $d_{(\lambda, l) \rightarrow(\mu, l+1)}^{(2 k)}=e_{2 k-2}^{(l)}$. Again the product $\varrho d_{(\lambda, l) \rightarrow(\mu, l)}^{(2 k+1)}=\varrho e_{2 k-2}^{(l)}$ is a single partition, of odd type $(k, l)$, and no power of $\delta$ occurs in the computation of the product. The diagram for this case is similar to Fig. 8, except that the lower vertex $\overline{k-l}$ of $\varrho$ is now an singleton block of $\varrho$.

Proposition 6.29 Let $(\lambda, l) \in \widehat{A}_{n}$ and $\mathfrak{s}, \mathfrak{t} \in \widehat{A}_{n}^{(\lambda, l)}$. Then $d_{\mathfrak{s}}^{*} c_{(\lambda, l)} d_{\mathfrak{t}}=d_{\mathfrak{s}}^{*} c_{(\lambda, 0)} e_{n-1}^{(l)} d_{\mathfrak{t}}$ lies in the $\mathbb{Z}$-span of $\mathcal{B}$.

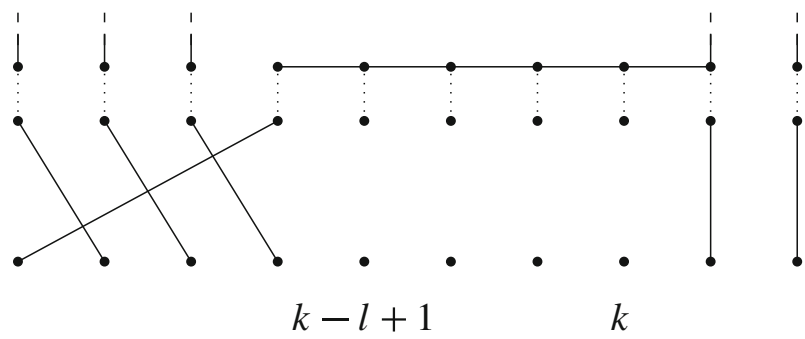

Fig. $7 \varrho s_{k-l, j} e_{2 k-1}^{(l)}$, where $\varrho$ is of odd type $(k+1, l+1)$ 


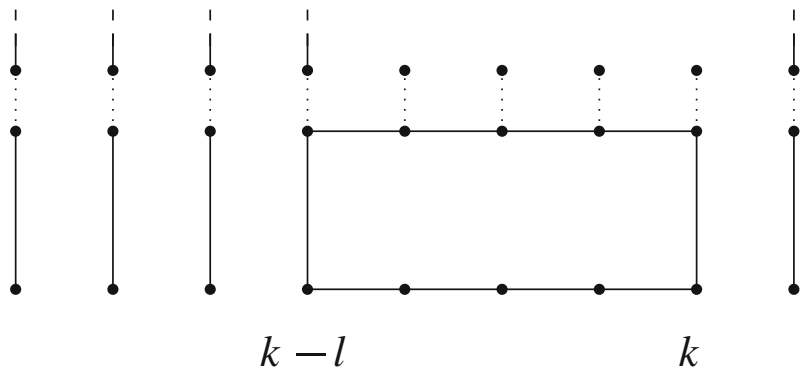

Fig. $8 \varrho^{\prime} e_{2 k-2}^{(l)}$, where $\varrho^{\prime}$ is of even type $(k, l)$

Proof If $n=2 k+1$ is odd, then $c_{(\lambda, 0)} e_{n-1}^{(l)}$ is a sum of partitions of odd type $(k+1, l)$. If $n=2 k$ is even, then $c_{(\lambda, 0)} e_{n-1}^{(l)}$ is a sum of partitions of even type $(k, l)$. The argument proceeds as in the proof of Proposition 6.12, with Lemma 6.28 taking the place of Lemma 6.11 .

Theorem 6.30 The set $\mathscr{A}_{n}=\left\{d_{\mathfrak{s}}^{*} c_{(\lambda, l)} d_{\mathfrak{t}} \mid \mathfrak{s}, \mathfrak{t} \in \widehat{A}_{n}^{(\lambda, l)},(\lambda, l) \in \widehat{A}_{n}\right\}$, is a basis for the partition algebra $A_{n}\left(R_{0} ; \delta\right)$ over the generic ground ring $R_{0}=\mathbb{Z}[\delta]$.

Proof The transition matrix $B$ between the diagram basis of the partition algebra and the set $\mathscr{A}_{n}$ has integer entries, according to Proposition 6.29 , and in particular $d=\operatorname{det}(B)$ is an integer. Since $\mathscr{A}_{n}$ is a basis for the partition algebra over $R=\mathbb{Z}\left[\boldsymbol{\delta}^{ \pm 1}\right]$, it follow that $B$ is invertible over $R$, so the integer $d$ is a unit in $R$. It follows that $d= \pm 1$ and hence $B$ is invertible over $\mathbb{Z}$. Hence $\mathscr{A}_{n}$ is a basis of the partition algebra over $R_{0}$.

Corollary 6.31 Let $A_{n}$ denote the partition algebra over the generic ground ring $R_{0}=$ $\mathbb{Z}[\boldsymbol{\delta}]$. For $n \geq 0$ and for $\Delta$ a cell module of $A_{n+1}$, the restricted module $\operatorname{Res}_{A_{n}}^{A_{n+1}}(\Delta)$ has an order preserving cell filtration.

Proof The proof is the same as that of Corollary 6.17.

Acknowledgments The first author is grateful to Arun Ram and Andrew Mathas for many stimulating conversations during the course of this research and for detailed comments on previous versions of this paper. The second author thanks Andrew Mathas for many helpful conversations and the School of Mathematics and Statistics at the University of Sydney for its hospitality. We thank Steffen König and the Institute for Algebra and Number Theory at the University of Stuttgart for hospitality. This research was supported by the Australian Research Council (grant ARC DP-0986774) at the University of Melbourne, (grants ARC DP-0986349, ARC DP-110103451) at the University of Sydney and EPSRC (grant EP/L01078X/1) at City University London.

Open Access This article is distributed under the terms of the Creative Commons Attribution 4.0 International License (http://creativecommons.org/licenses/by/4.0/), which permits unrestricted use, distribution, and reproduction in any medium, provided you give appropriate credit to the original author(s) and the source, provide a link to the Creative Commons license, and indicate if changes were made. 


\section{Appendix A. A Formula for Murphy Basis Elements}

In this appendix, we give an alternative formula for the Murphy basis of the Iwahori-Hecke algebra $\mathcal{H}_{n}\left(q^{2}\right)$ and for the Murphy-type bases of the various algebras treated in Section 6. The formula was pointed out to us by Chris Bowman for the case of the Hecke algebra, and Bowman posed the question whether an analogous formula holds also for the BMW algebras, etc.

Consider a tower $\left(H_{n}\right)_{n \geq 0}$ of cyclic cellular algebras satisfying the hypotheses of Section 3.5. As we will show, in all the examples of such towers treated in this paper, the elements $c_{\lambda}$ and the branching factors $d_{\mu \rightarrow \nu}^{(n+1)}$ and $u_{\mu \rightarrow \nu}^{(n+1)}$ can be chosen to satisfy

$$
c_{\mu} u_{\mu \rightarrow \nu}^{(n+1)}=\left(d_{\mu \rightarrow \nu}^{(n+1)}\right)^{*} c_{\nu}
$$

for all $n \geq 0$ and all $\mu \in \widehat{H}_{n}$ and $v \in \widehat{H}_{n+1}$ with $\mu \rightarrow v$.

We define an ordered product of $u$-coefficients along paths, analogous to the elements $d_{\mathfrak{t}}$ defined in Eq. (3.4).

Fix $n \geq 1$ and $\lambda \in \widehat{H}_{n}$. For each path $\mathfrak{t}=\left(\emptyset=\lambda^{(0)}, \lambda^{(1)}, \ldots, \lambda^{(n)}=\lambda\right) \in \widehat{H}_{n}^{\lambda}$, define

$$
u_{\mathfrak{t}}=u_{\emptyset \rightarrow \lambda^{(1)}}^{(1)} u_{\lambda^{(1)} \rightarrow \lambda^{(2)}}^{(2)} \cdots u_{\lambda^{(n-1)} \rightarrow \lambda^{(n)}}^{(n)} .
$$

Lemma A.1 Let $\left(H_{n}\right)_{n \geq 0}$ be a tower of cyclic cellular algebras satisfying the hypotheses of Section 3.5. Suppose that Eq. (A.1) holds for all $n \geq 0$ and all $\mu \in \widehat{H}_{n}$ and $\nu \in \widehat{H}_{n+1}$ with $\mu \rightarrow \nu$. Then for all $n \geq 0$, all $\lambda \in \widehat{H}_{n}$ and all $\mathfrak{t} \in \widehat{H}_{n}^{\lambda}$, one has

$$
d_{\mathfrak{t}}^{*} c_{\lambda}=u_{t}
$$

Consequently, the cellular basis of $H_{n}$ given in Corollary 3.11 can be written as

$$
\left\{u_{\mathfrak{s}} d_{\mathfrak{t}} \mid \lambda \in \widehat{H}_{n} \text { and } \mathfrak{s}, \mathfrak{t} \in \widehat{H}_{n}^{\lambda}\right\} .
$$

Proof The formula (A.3) is evident for $n=0$, 1. Fix $n \geq 1$ and suppose that (A.3) holds for all $\lambda \in \widehat{H}_{n}$ and all $\mathfrak{t} \in \widehat{H}_{n}^{\lambda}$. Let $v \in \widehat{H}_{n+1}$ and $\mathfrak{t}=\left(\emptyset, \lambda^{(1)}, \ldots, \lambda^{(n)}=\mu, \lambda^{(n+1)}=v\right)$ be an element of $\widehat{H}_{n+1}^{v}$. Write $\mathfrak{t}^{\prime}=\mathfrak{t}_{[0, n]}$. Then, using the induction hypothesis as well as Eq. (A.1), we have

$$
u_{\mathfrak{t}}=u_{\mathfrak{t}^{\prime}} u_{\mu \rightarrow v}^{(n+1)}=d_{\mathfrak{t}^{\prime}}^{*} c_{\mu} u_{\mu \rightarrow v}^{(n+1)}=d_{\mathfrak{t}^{\prime}}^{*}\left(d_{\mu \rightarrow v}^{(n+1)}\right)^{*} c_{v}=d_{\mathfrak{t}}^{*} c_{\nu} .
$$

The statement now follows by induction.

Lemma A.2 The branching factors $d_{\mu \rightarrow v}^{(n+1)}$ and $u_{\mu \rightarrow v}^{(n+1)}$ for the tower of Iwahori-Hecke algebras of the symmetric groups, as determined in 4.5 and Corollary 4.19, satisfy

$$
m_{\mu} u_{\mu \rightarrow v}^{(n+1)}=\left(d_{\mu \rightarrow v}^{(n+1)}\right)^{*} m_{v}
$$

for all $n \geq 0$ and all partitions $\mu$ of size $n$ and $\nu$ of size $n+1$ with $\mu \rightarrow v$.

Proof This is immediate from Lemma 4.18, part (1).

Corollary A.3 The Murphy basis of the Iwahori-Hecke algebra $\mathcal{H}_{n}\left(q^{2}\right)$ is given by

$$
m_{\mathfrak{s t}}^{\lambda}=u_{\mathfrak{s}} d_{\mathfrak{t}}
$$

for $\lambda$ a partition of $n$ and $\mathfrak{s}, \mathfrak{t}$ standard $\lambda$-tableaux. 
Proof This follows from Eq. (4.7) and Lemma A.1 and Lemma A.2.

Our next goal is to obtain similar formulas for the Murphy type bases of the various algebras treated in Section 6.

Proposition A.4 Let $A_{n}$ denote the n-th BMW, Brauer, partition or Jones-Temperley-Lieb algebra. The Murphy type basis of $A_{n}$ established in Section 6 can be written in the form

$$
\left\{u_{\mathfrak{s}} d_{\mathfrak{t}} \mid \lambda \in \widehat{A}_{n} \text { and } \mathfrak{s}, \mathfrak{t} \in \widehat{A}_{n}^{\lambda}\right\} .
$$

Sketch of proof. We need to show that if $x \in \widehat{A}_{n}$ and $y \in \widehat{A}_{n+1}$ with $x \rightarrow y$ in the branching diagram $\widehat{A}$, then

$$
c_{x} u_{x \rightarrow y}^{(n+1)}=\left(d_{x \rightarrow y}^{(n+1)}\right)^{*} c_{y},
$$

where the elements $c_{x} \in A_{n}$ and $c_{y}, u_{x \rightarrow y}^{(n+1)}, d_{x \rightarrow y}^{(n+1)} \in A_{n+1}$ are as specified in Section 6. The result will then follow from Lemma A.1. For the Temperley-Lieb algebras, Eq. (A.4) is evident from the formulas in Section 6.4 for the elements $c_{x}$ and for the branching factors.

For the BMW, Brauer and partition algebras, (A.4) can be established in two steps. The first step is to show that (A.4) holds when $x=(\lambda, 0) \in \widehat{A}_{n}$ and $y=(\hat{\mu}, 0) \in \widehat{A}_{n+1}$. For the Brauer and partition algebras this special case of (A.4) follows from Lemma 4.18, part (1), as all the elements involved lie in a copy of the symmetric group algebra contained in $A_{n+1}$. For the BMW algebras, it is necessary to establish an analogue of Lemma 4.18, part (1) which is valid in the algebra of the braid group.

The second step in the proof of (A.4) is to establish the general case from the special case. This involves a straightforward computation using the formulas of Theorem 5.7.

\section{References}

1. Birman, J.S., Wenzl, H.: Braids, link polynomials and a new algebra. Trans. Amer. Math. Soc. 313(1), 249-273 (1989)

2. Bowman, C., Enyang, J., Goodman, F.: Cellularity of diagram algebras and tensor space. in preparation (2016)

3. Bowman, C., Enyang, J., Goodman, F.: Diagram algebras, dominance triangularity, and skew modules. in preparation (2016)

4. Brauer, R.: On algebras which are connected with the semisimple continuous groups. Ann. Math. (2) 38(4), 857-872 (1937)

5. Dipper, R., James, G.: Representations of Hecke algebras of general linear groups. Proc. London Math. Soc. (3) 52(1), 20-52 (1986). MR 812444

6. Doran, IV W.F., Wales, D.B.: The partition algebra revisited. J. Algebra 231(1), 265-330 (2000). MR 1779601

7. East, J.: Generators and relations for partition monoids and algebras. J. Algebra 339, 1-26 (2011). MR 2811310

8. Enyang, J.: Cellular bases for the Brauer and Birman-Murakami-Wenzl algebras. J. Algebra 281(2), 413-449 (2004)

9. Enyang, J.: Specht modules and semisimplicity criteria for Brauer and Birman-Murakami-Wenzl algebras. J. Algebraic Combin. 26(3), 291-341 (2007)

10. Enyang, J.: A seminormal form for partition algebras. J. Combin. Theory Ser. A 120(7), 1737-1785 (2013)

11. Geetha, T., Goodman, F.M.: Cellularity of wreath product algebras and A-Brauer algebras. J. Algebra 389, 151-190 (2013)

12. Goodman, F.M.: Cellularity of cyclotomic Birman-Wenzl-Murakami algebras. J. Algebra 321(11), 3299-3320 (2009) 
13. Goodman, F.M., Graber, J.: Cellularity and the Jones basic construction. Adv. Appl. Math. 46(1-4), 312-362 (2011)

14. Goodman, F.M., Graber, J.: On cellular algebras with Jucys Murphy elements. J. Algebra 330, 147-176 (2011)

15. Goodman, F.M., Kilgore, R., Teff, N.: A cell filtration of the restriction of a cell module. preprint (2015), arXiv: 1504.02136

16. Graham, J.J., Lehrer, G.I.: Cellular algebras. Invent. Math. 123(1), 1-34 (1996)

17. Halverson, T., Ram, A.: Partition algebras. European J. Combin. 26(6), 869-921 (2005). MR 2143201

18. Härterich, M.: Murphy bases of generalized Temperley-Lieb algebras. Arch. Math. (Basel) 72(5), 337345 (1999)

19. Jones, V.F.R.: Index for subfactors. Invent. Math. 72(1), 1-25 (1983)

20. Jones, V.F.R.: The Potts model and the symmetric group. In: Subfactors (Kyuzeso, 1993), pp. $259-267$. World Sci. Publ., River Edge, NJ (1994)

21. Jones, V.F.R.: A polynomial invariant for knots via von Neumann algebras. Bull. Amer. Math. Soc. (N.S.) 12(1), 103-111 (1985)

22. Jost, T.: Morita equivalence for blocks of Hecke algebras of symmetric groups. J. Algebra 194(1), 201223 (1997)

23. König, S., Xi, C.: On the structure of cellular algebras. In: Algebras and modules, II (Geiranger, 1996), CMS Conf. Proc., vol. 24, pp. 365-386. Amer. Math. Soc., Providence, RI (1998)

24. König, S., Xi, C.: Cellular algebras: inflations and Morita equivalences. J. London Math. Soc. (2) 60(3), 700-722 (1999)

25. Martin, P.P.: The partition algebra and the Potts model transfer matrix spectrum in high dimensions. J. Phys. A 33(19), 3669-3695 (2000)

26. Martin, P.: Potts models and related problems in statistical mechanics, Series on Advances in Statistical Mechanics, vol. 5. World Scientific Publishing Co. Inc., Teaneck, NJ (1991)

27. Martin, P.: Temperley-Lieb algebras for nonplanar statistical mechanics - the partition algebra construction. J. Knot Theory Ramifications 3(1), 51-82 (1994)

28. Mathas, A.: Iwahori-Hecke algebras and Schur algebras of the symmetric group, University Lecture Series, vol. 15. American Mathematical Society, Providence, RI (1999)

29. Mathas, A.: Seminormal forms and Gram determinants for cellular algebras. J. Reine Angew. Math. 619, 141-173 (2008). With an appendix by Marcos Soriano.

30. Mathas, A.: A Specht filtration of an induced Specht module. J. Algebra 322(3), 893-902 (2009)

31. Morton, H.R., Wassermann, A.J.: A basis for the Birman-Wenzl algebra, unpublished manuscript (1989, revised 2000), 1-29, placed on the arXiv by H.R. Morton in 2010, arXiv:1012.3116

32. Murakami, J.: The Kauffman polynomial of links and representation theory. Osaka J. Math. 24(4), 745758 (1987)

33. Murphy, G.E.: The representations of Hecke algebras of type $A_{n}$. J. Algebra 173(1), 97-121 (1995)

34. Nazarov, M.: Youngs orthogonal form for Brauers centralizer algebra. J. Algebra 182(3), 664-693 (1996)

35. Okounkov, A., Vershik, A.: A new approach to representation theory of symmetric groups. Selecta Math. (N.S.) 2(4), 581-605 (1996)

36. Ram, A.: Seminormal representations of Weyl groups and Iwahori-Hecke algebras. Proc. London Math. Soc. (3) 75(1), 99-133 (1997)

37. Rui, H., Si, M.: Gram determinants and semisimplicity criteria for Birman-Wenzl algebras. J. Reine Angew. Math. 631, 153-179 (2009). MR 2542221

38. Stoll, F., Werth, M.: A cell filtration of mixed tensor space. Math. Z. 282(3-4), 769-798 (2016)

39. Vershik, A., Okounkov, A.: A new approach to the representation theory of the symmetric groups. II. J. Math. Sci. 131, 5471-5494 (2005)

40. Wenzl, H.: On the structure of Brauers centralizer algebras. Ann. Math. (2) 128(1), 173-193 (1988). MR 951511

41. Wilcox, S.: Cellularity of diagram algebras as twisted semigroup algebras. J. Algebra 309(1), 10-31 (2007). MR 2301230

42. Xi, C.: Partition algebras are cellular. Compos. Math. 119(1), 99-109 (1999). MR 1711582

43. Xi, C.: On the quasi-heredity of Birman-Wenzl algebras. Adv. Math. 154(2), 280-298 (2000). MR 1784677 